
leadleadleadleadleadleadleadleadleadleadleadleadleadleadleadleadleadleadleadleadleadleadleadleadleadleadleadleadleadleadl leadleadleadleadleadleadleadleadleadleadleadleadleadleadleadleadleadleadleadleadleadleadleadleadleadleadleadleadleadleadl leadleadleadleadleadleadleadleadleadleadleadleadleadleadleadleadleadleadleadleadleadleadleadleadleadleadleadleadleadleadl leadleadleadleadleadleadleadleadleadleadleadleadleadleadleadleadleadleadleadleadleadleadleadleadleadleadleadleadleadleadl leadleadleadleadleadleadleadleadleadleadleadleadleadleadleadleadleadleadleadleadleadleadleadleadleadleadleadleadleadleadl leadleadleadleadleadleadleadleadleadleadleadleadleadleadleadleadleadleadleadleadleadleadleadleadleadleadleadleadleadleadl leadleadleadleadleadleadleadleadleadleadleadleadleadleadleadleadleadleadleadleadleadleadleadleadleadleadleadleadleadleadl

\title{
LEAD AND MERCURY LEVELS IN GOLDEN AND BALD EAGLES AND ANNUAL MOVEMENTS OF GOLDEN EAGLES WINTERING IN EAST CENTRAL IDAHO 1990-1997
}

dleadleadleadleadleadh dleadleadleadleadleadl. dleadleadleadleadleadh dleadleadleadleadleadl. dleadleadleadleadleadl dleadleadleadleadleadl dleadleadleadleadleadl. dleadleadleadleadleadl. dleadleadleadleadieadlı dleadleadleadleadleadi

leadleadleadleadleadleadleadleadleadleadleadleadleadleadleadleadleadleadleadleadleadleadleadleadleadleadleadleadleadleadl leadleadleadleadleadleadleadleadleadleadleadleadleadleadleadleadleadleadleadleadleadleadleadleadleadleadleadleadleadleadi leadleadleadleadleadleadleadleadleadleadleadleadleadleadleadleadleadleadleadleadleadleadleadleadleadleadleadleadleadleadl leadleadleadleadleadleadleadleadleadleadleadleadleadleadleadleadleadleadleadleadleadleadleadleadleadleadleadleadleadleadl leadleadleadleadleadleadleadleadleadleadleadleadleadleadleadleadleadleadleadleadleadh - "laadleadleadleadleadleadleadleadl leadleadleadleadleadleadleadleadleadleadleadleadleadleadleadleadleadleadleadleadl 7 adleadleadleadleadleadleadlı leadleadleadleadleadleadleadleadleadleadleadleadleadleadleadleadleadleadleadlea d dleadleadleadleadlcadlcadl leadleadleadleadleadleadleadleadleadleadleadleadleadleadleadleadleadleadleadl tleadleadleadleadleadleadl leadleadleadleadleadleadleadleadleadleadleadleadleadleadleadleadleadleadlea $\{$ dleadleadleadleadleadleadl

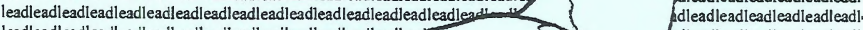
leadleadleadleadleadleadleadleadleadleadleadleadleadleadlo fadleadleadleadleadleadleadl leadleadleadleadleadleadleadleadleadleadleadleadlo eadleadleadleadleadleadleadl leadleadleadleadleadleadleadleadleadleadleadleadleadleadleadleadleadl leadleadleadleadleadleadleadleadlea fleadleadleadleadleadleadleadl leadleadleadleadleadleadleadlead 3 adleadleadleadleadleadleadleadl leadleadleadleadleadleadleadleg $\quad\{$ eadleadleadleadleadleadleadleadl leadleadleadleadleadleadleadleadleadleadleadleadleadleadleadleadl Icadleadleadleadleadlead adlcadlcadleadleadleadleadleadleadleadl leadleadleadleadleadlead alleadleadleadleadleadleadleadleadleadleadl leadleadleadleadleadleau cadleadleadleadleadleadleadleadleadleadleadlead! leadleadleadleadleadle _eadleadleadleadleadleadleadleadleadleadleadleadleadl leadleadleadleadlead dleadleadleadleadleadleadleadleadleadleadleadleadleadl leadleadleadleadlea of adleadleadleadleadleadleadleadleadleadleadleadleadleadl leadleadleadlead! head!s eadleadleadleadleadleadleadleadleadleadleadleadleadlead! leadleadleadley ady eadleadleadleadleadleadleadleadleadleadleadleadleadleadl leadleadlead Aeg Radieadlea leadhor adleadleadleadleadleadleadleadleadleadleadleadleadleadl leadleadleadeadleadleg pdleadleadle peadleadleadl leadleadlead1 f. pdleadleadl Ueadleadleadleadleadleadleadleadleadleadleadleadleadl leadleadleadieff leadleadlead vadleadleadleadleadleadleadleadleadleadleadlead leadleadleadlea fdleadleadlea $\quad$ Wleadleadleadleadleadleadleadleadleadleadleadl leadleadleadle leadle leadleadleadleadleadle leadleadleadleadleadle leadleadleadleadleadle

QI

84.2

.1352

no.

98-12 leadleadleadlea

eadleadlead"
BY: Erica H. and Tim H. Craig Western Ecological Studies Team P.O. Box 82

Tendoy, ID 83468

(208) $756-6356$

Idaho Bureau of Land Management

Technical Bulletin No. 98-12

October 1998 dleadleadleadleadleadleadl dleadleadleadleadleadleadl dleadleadleadleadleadleadl Yeadleadleadleadleadleadl eadleadleadleadleadleadl eadleadleadleadleadlead! 


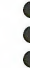




\section{LEAD AND MERCURY LEVELS IN GOLDEN AND BALD EAGLES AND ANNUAL MOVEMENTS OF GOLDEN EAGLES WINTERING IN EAST CENTRAL IDAHO \\ 1990-1997}

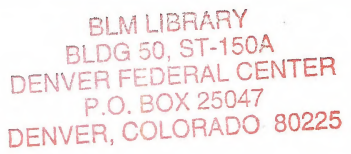

adleadleadleadleadleadleadleade a Aleadleadleadleadleadleadleadleadleadleadleadleadits leo

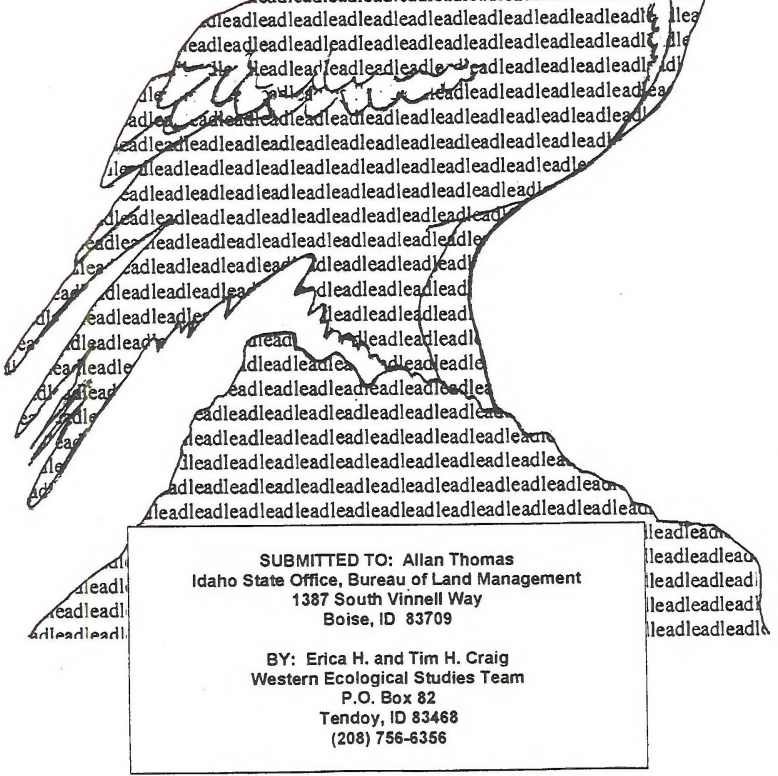




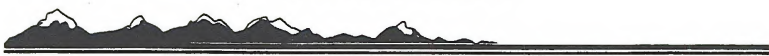

TITLE PAGE.

TABLE OF CONTENTS. .II

EXECUTIVE SUMMARY.

PHASE I. Objective: To determine lead levels in freeranging eagles in east central Idaho.

Work performed/results.

PHASE II. Objective: To determine the source of

lead contamination.

Work performed/results.

Management Implications for Phase I and II..

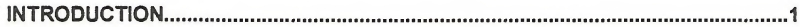

STUDY AREA

SECTION I. An Eight-year Study of Blood Lead and Mercury Levels in Wintering Golden and Bald Eagles in East Central Idaho; 1990-1997.

METHODS. .3

CAPTURE AND PROCESSING OF EAGLES...................................................

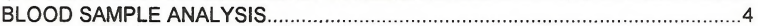

DATA ANALYSIS

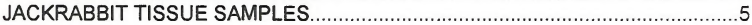

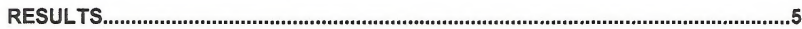

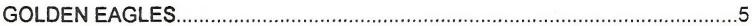

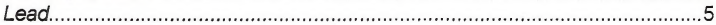

Recaptures/Sightings of Color-Marked Eagles.......................................... .6

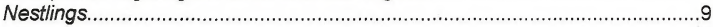

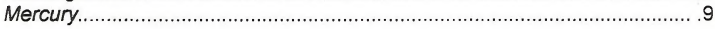

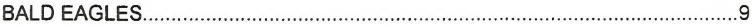

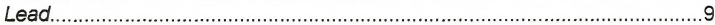

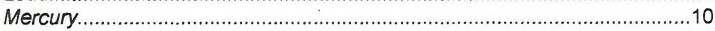

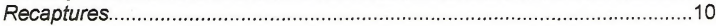


DISCUSSION .10

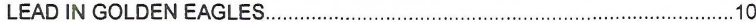

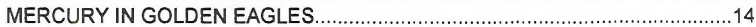

LEAD IN BALD EAGLES

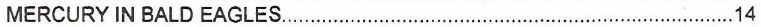

SECTION II. Satellite Telemetry of Golden Eagles

with Elevated Lead Levels.........................................................................................15

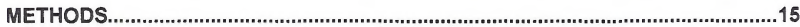

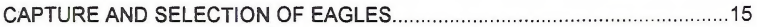

TELEMETRY VIA SATELLITE, DATA INTERPRETATION..................................16

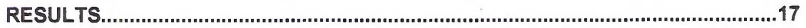

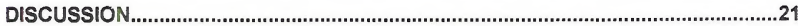

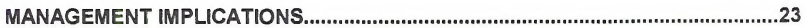

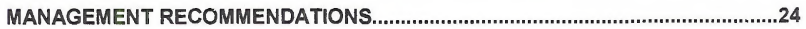

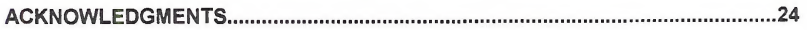

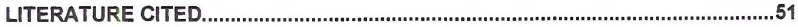

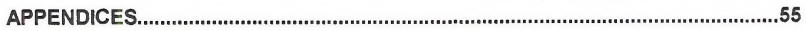

APPENDIX A

Duty Cycles of 6 golden eagles tracked via satellite in east central Idaho from January 1996 through

November 1997. Transmitter numbers identify the duty cycles of individual eagles.

APPENDIX B

Individual records of distance moved between location

estimates for 6 golden eagles tracked via satellite

telemetry during 1995-96. Distance moved since 
the previous location estimate is an average of all distances

in an individual day, for days on which more than

one location were received.

\section{APPENDIX C}

Monthly range maps of 6 golden eagles tracked via

satellite from winter 1995-96 through winter 1996-97.

Monthly range maps for adult, female golden eagle 5709;

January through March 1996

Monthly range maps for adult, male golden eagle 5727;

January through December 1996

Monthly range maps for adult, male golden eagle 5726;

December 1995 through December 1996

Monthly range maps for subadult, male golden eagle 5699;

January through May 1996.

Monthly range maps for subadult, male golden eagle 5738; January through November 1996. 1ن⿺辶

Monthly range maps for subadult, female golden eagle 5707 ; January 1996 through October 1996.

\section{TABLES}

SECTION I. An Eight-year Study of Blood Lead and Mercury Levels in Wintering Golden and Bald Eagles in East Central Idaho; 1990-1997.

Table 1. Mean blood lead levels (ppm, wet weight) of golden eagles captured in east central Idaho from January 1990 through

February 1997.

Table 2. Changes in blood lead levels of wintering golden eagles in east central Idaho recaptured one or more years after first capture; 1990-1997.

Table 3. Sex and age of wintering bald eagles captured in the

Lemhi and Pahsimeroi Valleys from 1990-1997 


\section{SECTION II. Satellite Tracking of Golden Eagles with Elevated Lead Levels}

Table 1. Description of classification and reliability of satellite location data (Service Argos, Inc., Landover, MD).

Table 2. Summary of telemetry transmission data, blood lead levels, sex and age of 6 golden eagles captured in east central Idaho and tracked by satellite from winter 1995-96 through winter 1996-97.

Table 3. Summary of telemetry data (convex polygons) for location classes $3-0$, based on data from 6 golden eagles from January 1996 through September 1997

\section{FIGURES}

SECTION I. An Eight-year Study of Blood Lead and Mercury Levels in Wintering Golden and Bald Eagles in East Central Idaho; 1990-1997.

Figure 1. Location of the winter study area for the eagle project in east central Idaho; 1990 through 1997

Figure 2. Comparison, by year, of blood lead levels in wintering golden eagles in east central Idaho; 1990-1997.

Figure 3. Age and sex of wintering golden eagles captured in east central Idaho; 1990-97.

SECTION II. Satellite Telemetry of Golden Eagles with Elevated Lead Levels.

Figure 1. Percentage of location estimates determined by satellite telemetry that fell in each of 7 different location classes. Data are presented by transmitter \# for each golden eagle monitored, as well as, for all location estimates combined.

Figure 2. Range of an adult female golden eagle (transmitter 5709 ) in the Intermountain West during winter 1995-96 
Figure 3. Locations for an adult female golden eagle (transmitter 5709) in the Intermountain West and Canada from winter 1995-96 through 7 April 1996.

Figure 4. Range of an adult male golden eagle (transmitter 5727 ) in the Intermountain West during winter 1995-96.

Figure 5. Locations for an adult male golden eagle

(transmitter 5727) in the Intermountain West during the spring

1996 migration period.

Figure 6. Home range of an adult male golden eagle (transmitter 5727 ) in the Intermountain West during breeding season 1996.

Figure 7. Locations for an adult male golden eagle (transmitter 5727) in the Intermountain West during fall migration 1996.

Figure 8. All locations for an adult male golden eagle (transmitter 5726 ) in east central Idaho from 30 December 1995 through 2 January 1997.

Figure 9. Range of an adult male golden eagle (transmitter 5726 ) in east central Idaho during winter 1995-96. 33

Figure 10. Locations for an adult male golden eagle

(transmitter 5726) in east central Idaho during spring migration 1996.

Figure 11. Home range of an adult male golden eagle

(transmitter 5726) in east central Idaho during breeding season 1996

Figure 12. Locations for an adult male golden eagle (transmitter 5726) in east central Idaho during fall migration 1996. .36

Figure 13. Range of a subadult male golden eagle

(transmitter 5699) in the Intermountain West during winter 1995-96.

Figure 14. Locations for a subadult male golden eagle (transmitter 5699) in the Intermountain West during spring migration 1996

Figure 15. Home range of a subadult male golden eagle (transmitter 5699) in the Intermountain West during breeding season 1996 
Figure 16. All locations for a subadult male golden eagle (transmitter 5699) in the Intermountain West from 18 January 1996 through 13 May 1996

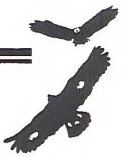

Figure 17. Range of a subadult male golden eagle (transmitter 5738) in the Intermountain West during winter 1995-96

Figure 18. Locations for a subadult male golden eagle (transmitter 5738) in the Intermountain West during spring migration 1996

Figure 19. Home range of a subadult male golden eagle (transmitter 5738) in the Intermountain West during breeding season 1996

Figure 20. Locations for a subadult male golden eagle (transmitter 5738) in the Intermountain West during fall migration 1996

Figure 21. All locations for a subadult male golden eagle (transmitter 5738) in the Intermountain West from 15 January 1996 through 3 January 1997.

Figure 22. Range of a subadult female golden eagle (transmitter 5707) in the Intermountain West during winter 1995-96.

Figure 23. Locations for a subadult female golden eagle (transmitter 5707) in the Intermountain West during spring migration 1996

Figure 24. Home range of a subadult female golden eagle (transmitter 5707) in the Intermountain West during breeding season 1996.

Figure 25. Locations for a subadult female golden eagle (transmitter 5707) in the Intermountain West during fall migration 1996.

Figure 26. All locations for a subadult female golden eagle (transmitter 5707) in the Intermountain West from 8 January 1996 through 26 November 1996. 


\section{EXECUTIVE SUMMARY}

PHASE I. Objective: To determine lead levels in free-ranging eagles in east central ldaho.

Work performed/results: Data were collected on eagles trapped from winter 1989-90 through winter 1996-97. Although we concentrated our trapping efforts primarily in the Lemhi and Pahsimeroi River Valleys, we also trapped birds in the Birch Creek Valley during winter 1996-97 because of historical accounts of lead contamination in that area (Oberg 1970). In addition, we collected a few blood samples from injured eagles that were turned in to the Idaho Department of Fish and Game during the course of the study.

In all, data were collected from 297 wintering golden eagles. Blood samples were analyzed for 290 of these birds. We also collected data from 39 wintering bald eagles and took blood samples from 36 of these birds.

Most $(99.6 \%$ ) golden eagles and $100 \%$ of the bald eagles sampled had detectable levels of lead in their blood (minimum detection limit: $0.01 \mathrm{ppm}$ ). Elevated blood lead levels $(\geq 0.20 \mathrm{ppm}$; Kramer and Redig 1997) were found in $45.6 \%$ of the wintering golden eagles and $61.1 \%$ of the bald eagles. There was no significant difference between the blood lead levels of golden eagles in the two major river drainages within the study area.

Although the number of golden eagles captured in the Birch Creek Valley $(n=5)$ was too small for statistical analysis, lead levels in these birds are similar to those in the other two valleys. All of the birds sampled in Birch Creek had detectable levels of lead in the blood and two of the five (40\%) had blood lead levels elevated above background.

Biologically incorporated lead (lead that has been ingested by prey animals and then incorporated in their tissues) is not thought to be a pathway of lead to raptors, at least, at higher levels of exposure (Pattee and Hennes 1983). Kramer and Redig (1997) and Pattee et al. (1990) found the highest incidence of lead contamination in eagles during the winter months and suggested that lead bullets, embedded in unretrieved game during hunting seasons, were the source of elevated lead levels in eagles. Nonetheless, because east central ldaho is an historic lead mining area in which lead contamination was a serious problem in the late $1800 \mathrm{~s}$, we could not ignore the possibility that lead contamination in eagles resulted from sources other than ingested shot or bullets.

PHASE II. Objective: To determine the source of lead contamination.

Work performed/results: We collected blood samples from 12 nestling golden eagles, just prior to fledging, in the Lemhi and Pahsimeroi Valleys in 1991 and 1992 . No lead contamination was detected in these birds. Therefore, we concluded that the prey fed 
these eaglets and which came from within their parents' home ranges, were not contaminated with lead.

Black tailed jackrabbits (Lepus californicus) are a major prey of eagles wintering in our study area (pers. observ.). To determine if biologically incorporated lead in the tissues of these prey was an avenue of lead to eagles, we collected jackrabbits in the winter of 1993-1994. Liver and muscle tissue samples were collected from jackrabbits in the Lemhi $(n=32)$ and Pahsimeroi $(n=29)$ Valleys, respectively. All of the liver samples were analyzed for lead but only muscle samples from jackrabbits with liver lead levels $\geq$ $0.50 \mathrm{ppm}$ were analyzed. Most, $(72.1 \%)$ of the jackrabbits sampled had liver lead levels below the detection limit and those with detectable lead in tissues had low levels. Research by Hoffman et al. (1981) on bald eagles suggests that the lead levels in jackrabbit tissues sampled in our study area are not high enough to elevate eagle blood lead levels above the detection limit.

In the winter of 1995-96 we fitted 6 golden eagles with satellite-monitored radiotransmitters, or Platform Transmitting Terminals (PTT). The satellite technology allowed us to obtain data on movements of the eagles across mountain ranges, as well as their migration patterns and year-round use areas. All 6 of the eagles we monitored had elevated blood lead levels. Tracking via satellite was continued for the life of the PTT battery, until the radio failed, or until the eagle was recaptured and the radio was removed.

The objectives of this tracking effort were to determine:

- if golden eagles with elevated blood lead levels were migrants or year round residents in our study area;

- the extent of interchange of golden eagles between the Lemhi and Pahsimeroi Valleys;

- the migration routes and summer and winter ranges of golden eagles with elevated blood lead levels;

- if there are common patterns of movements in golden eagles with elevated blood lead levels.

One of the 6 eagles we studied was a long-distance migrant whose radio failed in the spring following capture. This eagle was last located in April 1996 in central British Columbia, Canada. In contrast, another of our radio-tagged eagles was a year-round resident in the Lemhi Valley. The other four birds were "regional residents", ranging across portions of eastern Idaho and western Montana.

Interestingly, the only area used in common by all of these eagles was their winter range in our study area. It has been shown that golden eagles show annual fidelity to wintering and nesting areas (current study, Marzluff et. al. 1997, Brodeur et al. 1996). Therefore, it is probable that the eagles we studied made the same annual movements before and after we fitted them with PTT's. Consequently, these 6 eagles were probably all exposed to lead within the annual ranges we have identified for them. 
Furthermore, the eagle that remained in our study area year-round was likely exposed to lead within the Upper Lemhi Valley. In addition, the four eagles that were "regional residents" probably were exposed to lead in or near (within several hundred kilometers) our study area. The eagle that was a long-range migrant may have been exposed to lead anywhere on its winter range in Idaho, on its summer range, or along its migration route into Canada.

\section{Management Implications for Phase I and II.}

The telemetry data we collected showed that the annual ranges of the 6 golden eagles studied, include BLM administered lands throughout east central and southern Idaho, and Montana. Further, this study demonstrated that the golden eagle population in the study area comprises resident nesters, regional residents, and long distance migrants. If we assume that the movements of these birds after we began monitoring them were similar to their movements in the past, we can conclude that some contamination likely occurred locally. We do not know the specific source(s) of lead contamination in our study area. However, the area is used regularly by both species of eagles and many of them are contaminated with lead.

Most of the lead levels we found in eagles were sublethal. However, the specific effects on eagles of long-term, low-level exposure to lead are unknown. Approximately half of the eagles sampled were exposed to lead and our recapture data indicate that this exposure may be chronic. Further research is needed on the impact this chronic exposure may have on eagle populations over time. 


\section{INTRODUCTION}

Lead contamination has been reported in free ranging bald and golden eagles, sometimes in quantities that can cause mortality (Kramer and Redig 1997, Pattee et al. 1990, Reichel et al. 1984, Kaiser et al. 1980). Other researchers also have identified lead poisoning as a threat to raptors (Platt 1976, Hoffman et al. 1981, Pattee and Hennes 1983, USF\&WS 1985, Wiemeyer et al. 1989, Nelson et al. 1989, Pattee et al. 1990). Similarly, it has been suggested that long-term, low-level exposure to lead may weaken birds and affect reproduction, predispose birds to injury from environmental hazards or cause increases in predation, starvation and disease (Kramer and Redig 1997, Task Group on Metal Accumulation 1973, Pattee et al. 1981, Kendall 1982). Unfortunately, the effects of sublethal exposure to wildlife populations have been little studied (Pattee et al. 1981, Kendall 1982, Eisler 1988).

We began investigating lead $(\mathrm{Pb})$ contamination of eagles in Idaho during winter 198990 after finding incidents of $\mathrm{Pb}$ poisoning in bald and golden eagles in Idaho from 1977 through 1986 (Craig et al. 1990). The eagles reported in that study were all dead or moribund birds that had been turned in to the Idaho Department of Fish and Game from throughout the state. Five of 16 golden eagles and five of 6 bald eagles reported in that study were confirmed by the National Wildlife Health Research Center in Madison, Wisconsin to have died of lead poisoning. In addition, $83 \%$ of the bald eagles and $44 \%$ of the golden eagles had elevated concentrations of lead in their livers (Craig et al. 1990).

Data presented herein are the results of a subsequent eight-year study of lead and mercury levels in free-ranging golden eagles (Aquila chrysaetos), and bald eagles (Haliaeetus leucocephalus) that winter in east central Idaho. This research was divided into two different phases. Phase I of the study was to determine the extent of lead contamination in free ranging eagles that winter in east central Idaho. During this part of the study, we also monitored changes in blood lead levels in the sample eagle population, as well as, blood lead levels of individual eagles through time. Phase II of the study was to determine if the source of lead contamination to the wintering eagles in our study area originated locally. During this phase of the study, we sampled blood lead levels of nestling golden eagles and lead levels in tissues of eagle prey in the study area. We also monitored the movements of 6 golden eagles with elevated blood lead levels using radio telemetry via satellite from 1996-1997. The telemetry data allowed us to determine: 
- if golden eagles with elevated blood lead levels are migrants or year round residents in our study area;

- the extent that individual golden eagles move between the Lemhi and Pahsimeroi Valleys;

- the migration routes and summer and winter ranges of golden eagles with elevated blood lead levels;

- if there are common patterns of movements in golden eagles with elevated blood lead levels.

\section{STUDY AREA}

We trapped wintering golden and bald eagles within the adjacent Lemhi and Pahsimeroi River Valleys, parts of the Birch Creek Valley and nearby portions of the Salmon River Valley in Lemhi and Custer Counties, Idaho (Figure 1). The study area is bounded on the north by the Salmon River, the east by the Continental Divide and the west by the Lost River Mountain Range. The Lemhi mountains, which rise to over $3600 \mathrm{~m}$, separate the Lemhi Valley from the Pahsimeroi Valley. Both valleys range in elevation from approximately $1250 \mathrm{~m}$ to $2200 \mathrm{~m}$ and are each about $120 \mathrm{~km}$ in length. A hydrologic divide separates Birch Creek, which flows to the south, and the Lemhi River, which flows to the north.

The native habitat in the valleys and nearby foothills is typical of cool desert vegetatinn (Odum 1971). It is dominated by big sagebrush (Artemisia tridentata)-grass associations. This vegetation is interrupted in riparian areas by willow, Salix spp., birch, Betula spp. and cottonwood, Populus spp. Hay fields and irrigated pasturelands occur along the rivers and creeks, especially at lower elevations. Habitat in the mountains is mostly coniferous forests: predominantly Douglas fir, Pseudotsuga menziesii, and lodgepole pine, Pinus contorta. Rocky, snow-covered peaks occur above timberline.

Eagles that were radio-tagged in the study area and then tracked via satellite, ranged over parts of Idaho, Montana and British Columbia, Canada during the year after capture. 


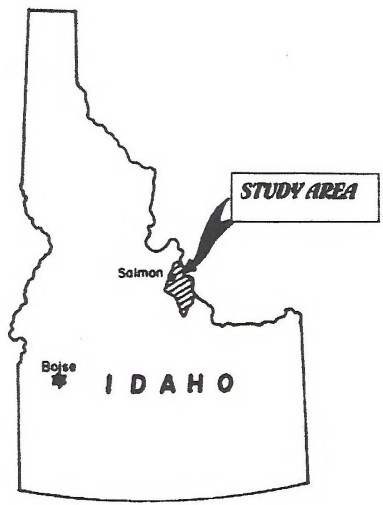

Figure 1. Location of the winter study area for the eagle project in east central Idaho; 1990 through 1997.

SECTION I. AN EIGHT-YEAR STUDY OF BLOOD LEAD AND MERCURY LEVELS IN WINTERING GOLDEN AND BALD EAGLES IN EAST CENTRAL IDAHO; 1990-1997.

\section{METHODS}

\section{CAPTURE AND PROCESSING OF EAGLES}

Wintering eagles were captured with padded leg-hold traps (after Bloom 1987), or occasionally, by hand. Samples also were collected from injured birds obtained from the Salmon Region of the Idaho Department of Fish and Game. Nestling eagles were captured just prior to fledging.

Captured birds were banded, measured (footpad, wing chord, tarsus, hallux, culmen and cranium) and photographed. Most birds were banded with white or silver USF\&WS bands on one leg and a numbered or alpha-numeric colored band (orange, yellow, or green) on the other. Five cc blood samples were collected by brachial vein puncture and then were placed in heparinized glass tubes and frozen at the end of each day. 


\section{BLOOD SAMPLE ANALYSIS}

Lead analysis of blood samples was done at the Holm Research Center at the University of Idaho in Moscow, Idaho for 6 of the 7 winters of the study. Analysis at the Holm Research Center was done with a Perkin-Elmer Zeeman 5011 PC Atomic Adsorption Spectrophotometer graphite furnace at a wavelength of $283.3 \mathrm{~nm}$.

Lockheed Martin, a Lockheed Idaho Technologies Company in Idaho Falls, Idaho analyzed all golden eagle blood samples collected during winter 1995-96. In order to place PTT's on eagles with elevated blood lead levels and still release them quickly, it was necessary to analyze blood samples on the same day eagles were captured. Lockheed Martin is the closest facility capable of the lead analysis we required. They determined lead levels with a VG Elemental PlasmaQuad through inductively coupled plasma-mass spectroscopy (ICP-MS). A wavelength of $207.97 \mathrm{~nm}$ was used for lead, and an internal standard of indium was used at a wavelength of $114.9 \mathrm{~nm}$. The lower limit of reportable residues was $0.01 \mathrm{ppm}$ (wet weight) for lead in all samples.

Golden eagle blood samples were analyzed for $\mathrm{Hg}$ levels through the winter of 199495. Bald eagle bloods were analyzed for $\mathrm{Hg}$ every year of the study. Total mercury was analyzed by ICP atomic emission in a hydrogen-saturated atmosphere on the Perkin-Elmer P-40 or Leeman 2000. The lower limit of reportable residues was 0.001 $\mathrm{ppm}$ (wet weight) for $\mathrm{Hg}$.

\section{DATA ANALYSIS}

All eagles were classified as either: subadult female, subadult male, adult female and adult male for statistical analysis. We did not take a second blood sample from eagles recaptured within five days of first capture. However, calculations of age and sex ratios were based on all birds captured, including these short-term recaptures. Therefore, the sample sizes for age and sex ratios are larger than those used in analysis of blood lead data. In addition, data from recaptured birds were not used in ANOVA tests because the samples were not necessarily independent of one another.

Blood lead values in eagles were sorted into four categories for analysis: $<0.20 \mathrm{ppm}=$ background; $0.20 \mathrm{ppm}-0.60 \mathrm{ppm}=$ exposed; $0.61-1.20=$ clinically affected; $>1.20$ $\mathrm{ppm}=$ acute lead poisoning (Kramer and Redig 1997).

For statistical tests requiring lead concentration data that are normally distributed, values were multiplied by 1000 and then log-transformed prior to analysis. Samples with lead values below detection were analyzed using one-half the minimum detection level (after Pattee et al. 1990). All means reported in the data are arithmetic means unless otherwise stated. The winter golden eagle blood lead data for the Pahsimeroi and Lemhi Valleys were examined using a two (valleys) by two (sexes) by 7 (years) 
fixed effects ANOVA model. Data from recaptured eagles were excluded in this analysis. Recapture data were analyzed using a paired t-test.

\section{JACKRABBIT TISSUE SAMPLES}

Black-tailed jackrabbits (Lepus californicus), the major live prey of eagles wintering in our study area (pers. observ.), were collected during the winter of 1993-94. Jackrabbit liver and muscle tissue samples ( $\geq 5 \mathrm{gms}$ each) were collected with steel shot from the Lemhi and Pahsimeroi Valleys. The samples were frozen until analyses were completed at the Holm Research Center, University of Idaho in Moscow, Idaho. Minimum detection level for tissue samples was $0.05 \mathrm{ppm}$ wet weight. All of the liver samples were analyzed for lead but only muscle samples from jackrabbits with liver lead levels $\geq 0.50 \mathrm{ppm}$ were analyzed.

\section{RESULTS}

\section{GOLDEN EAGLES}

Lead. From January 1990 through February 1997, data were collected from 290 wintering golden eagles. Lead was detected in the blood of $99.6 \%$ of the 281 eagles we trapped and from which blood samples were taken. The mean blood lead concentration of all the wintering golden eagles captured was $0.26 \mathrm{ppm} \pm 0.35 \mathrm{ppm}$ lead in blood (geometric mean: $0.17 \mathrm{ppm}$; range: $3.00 \mathrm{ppm}$ - below the detection limit). Elevated blood lead levels ( $\geq 0.20 \mathrm{ppm}$ ) were found in $45.6 \%$ of the wintering birds sampled. Of these eagles, most were sub-clinically affected $(0.20-0.60 \mathrm{ppm}$; Figure 2). However, a small group (5.4\%) of birds had blood lead levels in the two highest categories ( $>0.60 \mathrm{ppm}$ lead).

Although $52 \%$ of the golden eagles sampled in the Lemhi Valley and only $37 \%$ of the eagles captured in the Pahsimeroi Valley had elevated blood lead levels, there was no significant difference in golden eagle blood lead levels between the two valleys $(P=0.351)$. The sample obtained $(n=5)$ from the Birch Creek Valley was too small for statistical analysis. However, lead levels in golden eagles trapped there appeared similar to those in the other two valleys. All of the birds sampled in the Birch Creek Valley had detectable levels of lead in the blood and two of the five $(40 \%)$ had blood lead levels elevated above background.

There was a highly significant difference in golden eagle blood lead levels among years $(P=0.000$; Table 1$)$. Also, there was a significant valley by age interaction $(P=0.030)$; more subadult males $(39.5 \%)$ were captured in the Pahsimeroi than any other age class of eagle (Figure 3 ). More subadult golden eagles were captured in the study area than adults and more males than females but neither of these differences were significant ( $P=0.488$ and $P=0.689$, respectively). 
Table 1. Mean blood lead levels (ppm, wet weight) of golden eagles captured in east central Idaho from January 1990 through February 1997.

\begin{tabular}{cccc}
\hline Year & $\mathbf{n}$ & Arithmetic mean & Geometric mean \\
\hline $1989-90$ & 14 & 0.42 & 0.31 \\
$1990-91$ & 16 & 1.10 & 0.11 \\
$1991-92$ & 34 & 0.14 & 0.10 \\
$1992-93$ & 42 & 0.20 & 0.16 \\
$1993-94$ & 33 & 0.41 & 0.21 \\
$1994-95$ & 34 & 0.16 & 0.08 \\
$1995-96$ & 32 & 0.31 & 0.24 \\
$1996-97$ & 33 & 0.32 & 0.28 \\
\hline
\end{tabular}

Recaptures/Sightings of Color-marked Eagles. We recaptured $(n=39)$ or recovered dead $(n=4), 43$ golden eagles during the winters from 1991-1997. Four of the eagles we recaptured were caught the second time during the same winter in which they were first caught. Fifteen were recaptured the winter following first capture. Eight were recaptured two years later and two of these birds had originally been banded as nestlings in the study area. Eight birds were recaptured three winters later. Three birds were recaptured four winters after first capture (one of these birds was first banded as a nestling in the study area), and one was recaptured five winters later. Most of the recaptured birds were retrapped only once. However, three were recaptured twice and two golden eagles were recaptured three times.

Most of the golden eagles we captured the second time were at, or within a mile of, the original capture site. However, two of the eagles were recaptured in the valley adjacent to the valley in which they were first caught.

In addition to recapturing birds we banded, we also captured one golden eagle on 6 December 1994 that was originally banded as a nestling by another biologist on the Egaksrak River in the Arctic National Wildlife Refuge on 17 July 1990 (C. Mclntyre pers. comm.) Interestingly, another eagle that was captured and equipped with a PTT as a nestling in Denali National Park, Alaska in 1991 spent part of the following winter in the Lemhi Valley (C. Mclntyre, pers. comm.).

The causes of mortality for birds that were recovered dead, were: electrocution (2), collision with an automobile (1), and undetermined poisoning (1). One banded eagle was recaptured alive but died of secondary sodium pentothal poisoning shortly after capture.

There was not a significant difference in blood lead levels of individual golden eagles between the first and last capture $(n=33 ; p=0.091)$. Blood lead levels increased in $58.8 \%$ of the recaptured eagles and decreased or stayed the same in $41.1 \%$ of the birds (Table 2). 
Figure 2. Comparison, by year, of Blood Lead Levels in Wirtering Golden Eagles in East Central Idaho; 1990-97.

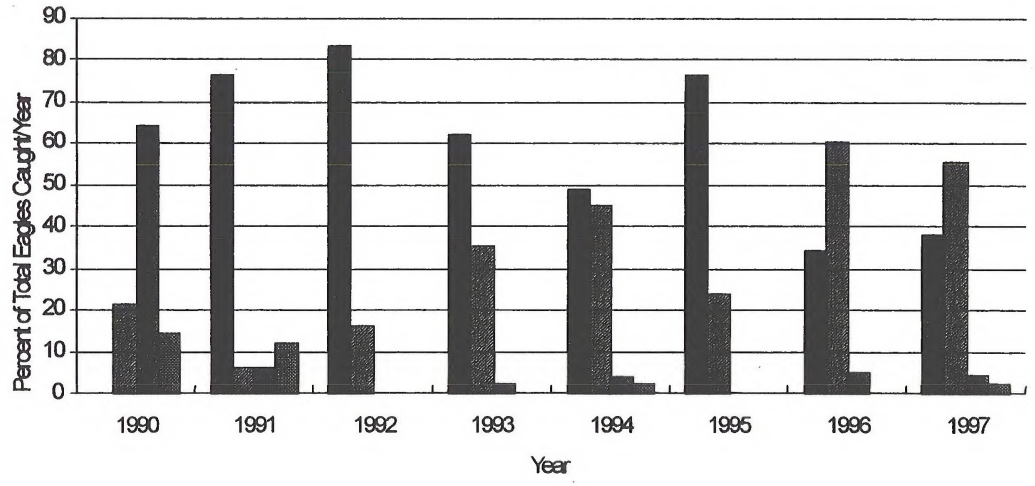

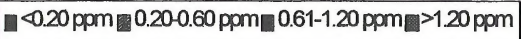


Figure3. Age and Sex of Wintering Golden Eagles Captured in East Central Idaho; 1990-97.

\section{Lemhi Valley}

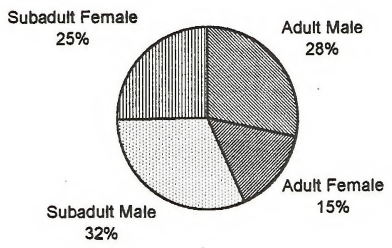

\section{Pahsimeroi Valley}

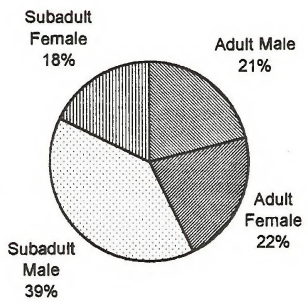


Table 2. Changes in blood lead levels of wintering golden eagles in east central Idaho recaptured one or more years after first capture; 1990-1997.

\begin{tabular}{llllll}
\hline $\begin{array}{l}\text { Change in Blood } \\
\text { Lead Levels }\end{array}$ & -1 & - & 0 & + & +1 \\
\hline sample size & 5 & 7 & 2 & 9 & 11 \\
$\begin{array}{l}\text { mean \# years } \\
\text { between samples }\end{array}$ & 1.6 & 2.3 & 2.0 & 2.1 & 2.5 \\
\hline
\end{tabular}

Key: -1: lead level decreased by one exposure category (see methods)

-: decrease in lead level but remained in same category

0 : no change in lead level

+ : increase in lead level but remained in same category

+1: lead level increased by one exposure category

Nestlings. Twelve blood samples were obtained in summer 1991 and 1992 from nestling golden eagles, just prior to fledging. None of these bloods contained elevated lead.

Mercury. None of the golden eagles sampled from 1990-1995 in our study had elevated $\mathrm{Hg}$ levels in the blood $(\overline{\mathrm{x}}=0.027 \pm 0.047)$. Therefore, mercury analysis of golden eagle bloods was discontinued after winter 1994-95.

\section{BALD EAGLES}

Lead. Data were collected from 39 bald eagles during the study. Blood samples were taken from 36 of these birds. Although an equal number of adult and subadult bald eagles were captured, most were males (Table 3 ). Most of the bald eagles were assumed to be nonresidents because there are only three known bald eagle nesting territories within the study area.

Table 3. Sex and age of wintering bald eagles captured in the Lemhi and Pahsimeroi Valleys from 1990-1997.

\begin{tabular}{cccccc}
\hline & $\begin{array}{c}\text { ADULT } \\
\text { MALE }\end{array}$ & $\begin{array}{c}\text { ADULT } \\
\text { FEMALE }\end{array}$ & $\begin{array}{c}\text { SUBADULT } \\
\text { MALE }\end{array}$ & $\begin{array}{c}\text { SUBADULT } \\
\text { FEMALE }\end{array}$ & TOTAL \\
\hline sample size & 15 & 3 & 13 & 5 & 36 \\
Percent & 41.7 & 8.3 & 36.1 & 13.9 & 100.0 \\
\hline
\end{tabular}

The mean blood $\mathrm{Pb}$ level for bald eagles was $0.43 \pm 0.46 \mathrm{ppm}$ (geometric mean: 0.26 ppm ; range: $1.90-0.04 \mathrm{ppm})$. Fourteen (38.9\%) of the bald eagles had blood lead 
levels $\leq 0.20 \mathrm{ppm} ; 15$ (41.7\%) had blood lead levels from $0.20-0.60$; five (13.9\%) had blood lead levels from $0.61-1.20 \mathrm{ppm}$; and $2(5.5 \%)$ had $\geq 1.20 \mathrm{ppm}$ lead in blood.

Mercury. The mean level of $\mathrm{Hg}$ in the sampled bald eagles was $1.10 \pm 0.63 \mathrm{ppm}$ (geometric mean: 0.94 ppm; range: $2.70-0.04$ ppm).

Recaptures. We recaptured one adult male bald eagle. This bird was recaptured within two days of first capture, so no additional blood samples were collected.

One subadult male bald eagle caught on 9 January 1993 in the Pahsimeroi Valley, was found dead in Alberta, Canada on 30 April 1993. This bird's blood lead level when captured was $0.34 \mathrm{ppm}$ and its $\mathrm{Hg}$ level was $0.55 \mathrm{ppm}$. No information was available on the cause of death or on $\mathrm{Pb}$ and $\mathrm{Hg}$ levels in the eagle at the time of its death.

A subadult female bald eagle banded on 12 January 1996 near Lemhi, Idaho in the Lemhi Valley was found electrocuted 17 May 1996 approximately $61 \mathrm{~km}$ southeast of Salmon, Idaho. Her blood lead level in January was in the exposed category $(0.23 \mathrm{ppm}$ lead) and there were $1.10 \mathrm{ppm} \mathrm{Hg}$ in the blood. No information is available currently on $\mathrm{Pb}$ or $\mathrm{Hg}$ levels in this eagle at the time of death.

\section{LEAD IN JACKRABBITS}

Liver and muscle tissue samples were collected from 32 jackrabbits in the Lemhi Valley and 29 jackrabbits in the Pahsimeroi Valley during winter 1993-94. Seventy-two percent of all the jackrabbits sampled had liver lead levels below the detection limit. In the Pahsimeroi River Valley $24.1 \%$ (7) of the jackrabbits had detectable lead levels in the liver (range 0.29 $-2.10 \mathrm{ppm}$ ). Of these muscle samples analyzed, only one had detectable lead (0.97 ppm). In the Lemhi Valley $31.3 \%(10)$ of the jackrabbits had detectable lead in their livers (range: $0.29-0.79 \mathrm{ppm}$ ). No jackrabbit muscle tissues sampled in the Lemhi River Valley

contained detectable lead.

\section{DISCUSSION}

\section{LEAD IN GOLDEN EAGLES}

The number of wintering golden eagles in our study area with elevated blood lead levels from 1990-1997 (45.6\%) is slightly higher than reported by Pattee et al. (1990) in southern California (35.8\%; $n=162)$. Another study on the prevalence of lead exposure and/or lead poisoning in bald and golden eagles from eleven states, ranged from 3-44\% over a 16-year period; $1980-1995$ (Kramer and Redig 1997). A study of migrating golden eagles in Montana reported a higher incidence of lead contamination than we found, with $56 \%$ of 86 golden eagles having blood lead levels $>0.20 \mathrm{ppm}$ wet 
weight (Harmata and Restani 1995). These data suggest that the sources of lead contamination to golden eagle populations are widespread.

The connection between lead-shot used in waterfow hunting and lead poisoning in bald eagles has been well documented (Reichel et al. 1984, Feierabend and Myers 1984). Because of this link, it was expected that the number of lead-poisoned eagles would decrease after the 1991 federal ban on the use of lead shot in waterfowl hunting. Unfortunately, neither we nor Kramer and Redig (1997) have observed a decline in lead-poisoned eagles since 1991. Although we found a significant difference in lead levels among years, there has been no downward trend during our study. In fact, the number of eagles captured that had elevated lead in their blood during the winters of 1995-96 and 1996-97, is higher than in the previous four winters. This suggests that there is a continuing source of lead contamination to eagles. Kramer and Redig (1997) suggest that we should reevaluate current theories regarding the source of lead contamination in eagles, as well as, how eagles are poisoned.

The physiological conditions that affect the uptake, binding and release of $\mathrm{Pb}$ by tissues and organs in animals are not well known (Task Group on Metal Accumulation 1973). In addition, lead accumulations following ingestion of $\mathrm{Pb}$ shot can be affected by many factors, including age, geographic location, habitat, and time of year (Eisler 1988). Nonetheless, blood lead levels are thought to be an accurate indication of relatively recent exposure to lead. Lead in blood is available for distribution throughout the body, absorption into other tissues, or can be excreted from the body (Task Group on Metal Accumulation 1973). As this occurs, in the absence of a continued source of contamination, lead levels in the blood tend to decrease. Kendall et al. (1982) reported blood lead levels of a ringed turtle-dove (Streptopelia risoria) fed a single oral dose of 2 lead pellets to have dropped from $4.69 \mathrm{mg} / \mathrm{l}$ at 24 hours after ingestion, to $0.14 \mathrm{mg} / \mathrm{l}$ at 14 days after ingestion. We assume that blood lead levels in eagles would show a similar decline in the absence of new sources of lead contamination. However, our results from individual eagles tracked over a long period did not necessarily show a decrease in blood lead levels. In fact, birds recaptured at least one winter apart exhibited no significant difference in blood lead levels between first and last recapture. Pattee et al. (1990) also reported no significant difference in the blood lead levels of golden eagles based on age. In our study, although some golden eagle blood lead levels decreased between subsequent recaptures, blood lead concentrations increased with age in $58.8 \%$ of the golden eagles recaptured. This indicates that many of the eagles we sampled must be repeatedly exposed to lead contamination.

Biologically incorporated or tissue-bound lead has been recognized as a possible contributing factor to elevated lead levels in raptors but it is not generally considered to be the primary source of lead toxicosis (Pattee and Hennes 1983). Henny et al. (1991, 1994) have reported that lead in the tissue of prey is not readily available to raptors because it generally concentrates in bones, which are not completely digested. In addition, eagles often strip the flesh from prey and leave much of the bony material behind (pers. observ.). 
In any event, few jackrabbits sampled in our study area contained elevated levels of lead in tissue and those that did, contained low concentrations (Craig and Craig 1995). Research by Hoffman et al. (1981) on bald eagles suggests that the lead levels in jackrabbit tissues sampled in our study area are not high enough to elevate eagle blood lead levels above the detection limit. This supports the contention that biologically incorporated lead in prey is not the likely avenue of lead contamination to the eagles wintering in our study area. These data also support our finding of no difference in lead levels among the valleys, in spite of the history of lead mining in the Lemhi and Birch Creek Valleys.

The source of lead contamination in raptors is usually thought to be from imbedded lead shot or bullets ingested with the tissues of crippled or dead animals (Hoffman et al. 1981, Pattee et al. 1990, Redig 1980, Eisler 1988). Researchers have found significant differences in mean lead values by month for golden eagles, with the highest values occurring during the winter months and the lowest during the summer (Kramer and Redig 1997, Pattee et al. 1990). Kramer and Redig (1997) also reported that the highest incidences of lead poisoning were during the months of November and December. Similarly, the National Wildlife Health Research Center reported that $88 \%$ of the bald eagles diagnosed as having died of lead poisoning were collected from November through March (Anonymous 1992). Although there are few direct data to support the assumption, elevated lead levels in golden and bald eagles during the winter months are often attributed to lead projectiles in the carcasses of hunter-killed or crippled animals (Platt 1976, Pattee and Hennes 1983, Nelson et al. 1989, Pattee et al. 1990).

The eagles in our study area congregate in places where black-tailed jackrabbits are found in the winter. Hunting these hares is a popular winter pastime for people.

Eagles often feed on jackrabbits shot and left by hunters (pers. observ.). We have also observed bald and golden eagles feeding on big game animals during the winter, some of which probably died after having been wounded by hunters earlier in the fall.

Published accounts of big game deaths due to loss of wounded animals range from 6,9 and $10 \%$ in Montana (Pac et al. 1995) to $27 \%$ in northern Idaho (Unsworth et al. 1993). Big game hunting seasons occur during the winter in our study area and golden eagles have been observed to congregate in the areas of these hunts (M. Scott, IDF\&G, pers. comm.). We assume that these eagles were feeding on carrion associated with big game hunts and that this is a possible source of lead contamination to eagles within our study area during the winter. Lead projectiles are nearly always used in big game and black-tailed jackrabbit hunting in east central Idaho (pers. observ.). Kramer and Redig (1997) report that lead contamination has persisted in eagles even after the 1991 ban of lead shot in waterfowl hunting. They suggest that lead contamination might be related to the year-round hunting of small mammals and birds and/or offal containing lead fragments that remain in the field during big game hunting seasons. 
It is known that free-ranging eagles die from $\mathrm{Pb}$ poisoning (Pattee et al. 1981, Redig 1993 Reichel et al. 1984, Craig et al. 1990, Weimeyer et al. 1989, Anonymous 1992, Franson et al. 1995) and that high blood lead levels are indicative of acute exposure to lead (Hoffman et al. 1981, Pattee et al. 1981). However, blood lead levels of most of the golden eagles we have sampled, as well as, those reported by other researchers (Kramer and Redig 1997, Pattee et al. 1990), are in the lowest category of exposure (0.20-0.60 ppm). These lead levels are not likely to be a direct cause of death. However, the effects of this long-term, low-level exposure to $\mathrm{Pb}$ on free-ranging eagles is unknown.

Predicting the effects of lead exposure on free-ranging animals is complicated because survival and successful reproduction are influenced by many variables. For example, high absorption rates for calcium (as might occur during egg laying) may increase the absorption of lead. In addition, there is considerable variation in the responses of different avian species to lead exposure (Beyer et al. 1988). However, research on Rock doves (Columba livia) showed that chronic exposure to lead interfered with learning behavior (Dietz et al. 1979). If lead exposure affects learning in golden eagles, long-term contamination could adversely affect their survival rates. Similarly, exposure to other contaminants, susceptibility to disease, cold-stress, accidental injury and starvation can all contribute to the death or reproductive failure of an individual already weakened by lead exposure (Kramer and Redig 1997, Kendall 1982, Pattee et al. 1981, Task Group on Metal Accumulation 1973).

We are unaware of controlled laboratory studies on the effects of lead ingestion on golden eagles. However, there have been laboratory studies on the effects of $\mathrm{Pb}$ on bald eagles (Pattee et al. 1981, Hoffman et al. 1981). Pattee et al. (1981) found that the individual responses of five bald eagles experimentally dosed with \#4 lead shot were quite variable. For example, four of the eagles died from 10 to 125 days after the experiment began. The fifth experimental eagle did not die from lead poisoning although it was fed $80 \# 4$ lead shot pellets during the course of the experiment. Eventually, this bird became blind and was finally sacrificed after 135 days. A subsequent necropsy revealed that $129.0 \mathrm{mg}$ of the lead had been eroded by digestion during the study. Nonetheless, lead levels in liver and kidney were low, $3.2 \mathrm{ppm}$ and 3.4 ppm, respectively. Pattee et al. (1981) concluded that sublethal exposure to lead also can be harmful to bald eagles and can indirectly contribute to their death.

Reports on the effects of $\mathrm{Pb}$ on reproduction in raptors have been conflicting. Henny et al. (1994) found reduced reproductive rates in American kestrels (Falco sparverius) that nested in a study area contaminated with lead. However, the population-wide productivity of the birds was not significantly different than in a control area. In the same study area, Henny et al. (1991) reported no decrease in overall productivity in an osprey population, although some of the individual birds had elevated lead levels. However, Henny et al. (1991) did not report on the productivity of individual osprey with elevated lead levels. It is interesting to note that the golden eagle with the highest blood lead levels (0.71 ppm) in our study did not nest successfully in 1996. 
Elevated blood lead levels have been reported in $30 \%$ to $50 \%$ of the golden eagles in sampled populations in the western U.S. (this study, Pattee et al. 1990, Harmata and Restani 1995). If sublethal levels of lead have a negative impact on the productivity or survival of golden eagles, then there is the potential for population-wide impacts on golden eagles through time. This may be increasingly significant when considered with the long-term downward trends in golden eagle numbers in the western United States reported by Hoffman et al. (1996).

\section{MERCURY IN GOLDEN EAGLES}

The importance of elevated $\mathrm{Hg}$ levels in tissues of eagles is not fully understood. Mercury accumulation in the environment is generally related to aquatic systems (Eisler 1987 ) and the source of $\mathrm{Hg}$ to raptors is generally aquatic prey. Although golden eagles in the West have been reported to prey upon fish (Brown 1992), their prey are generally mammalian and avian species in upland habitats (pers. observ., Eakle and Grubb 1986). Thus, they are less likely to be exposed to mercury contamination. The golden eagles in our study did not contain elevated mercury levels, and in most samples, mercury blood lead levels were below the detection limit. Consequently, we discontinued mercury analysis of blood samples from golden eagles after winter 19941995.

\section{LEAD IN BALD EAGLES}

The sample population of bald eagles wintering in our study area had higher mean lead levels in blood (both arithmetic and geometric means) than the wintering golden eagles sampled. In addition, more wintering bald eagles sampled had elevated blood lead levels than did wintering golden eagles sampled. A spring study of migrating eagles in Montana, (Harmata and_Restani 1995) also reported_higher blood lead levels in bald than in golden eagles $(\bar{x}=0.32 \mathrm{ppm}$ wet weight and $\bar{x}=0.18 \mathrm{ppm}$ wet weight, respectively; geometric means not given). Mean blood lead level of bald eagles in our study area was slightly higher than reported in the Montana study (comparison based on arithmetic means).

The difference in lead levels in bald and golden eagles may result from differences in the food or feeding habits of the two species. Bald eagles are more likely to feed on prey associated with aquatic ecosystems (Terres 1980) and thus be exposed to lead contamination (Reichel et al. 1984, Feierabend and Myers 1984).

\section{MERCURY IN BALD EAGLES}

Mercury levels in the blood of bald eagles wintering in our study area were much higher than mercury levels in golden eagles. Wiemeyer et al. (1989) has noted that bald eagles routinely have higher mercury concentrations in their blood than other species of birds. Again, this difference is probably related to dissimilarities in prey between the 
two species of eagles. Harmata and Restani (1995) also found that mercury levels were higher in vernal migrant bald than in golden eagles in Montana. However, mean blood mercury concentrations in the bald eagles in our study were higher than reported for bald eagles in the Montana study (arithmetic mean: $0.54 \mathrm{ppm}$; Harmata and Restani 1995). Conversely, the values from our study area were less than those reported for 15 bald eagles from Oregon, northern California and Montana (> 3 ppm mercury in blood; Wiemeyer 1989). Little is known about the relationship of mercury concentrations in blood and exposure rates and their subsequent health consequences (Wiemeyer 1989).

\section{SECTION II. SATELLITE TRACKING OF GOLDEN EAGLES WITH ELEVATED LEAD LEVELS}

\section{METHODS}

\section{CAPTURE AND SELECTION OF EAGLES}

During winter 1995-1996 three male and three female golden eagles with elevated lead levels were captured and fitted with Platform Transmitter Terminals (PTTs) from Microwave Telemetry Inc. (Columbia, MD). The PTTs were attached with teflon ribbon in a backpack configuration (similar to Snyder et al. 1989). Each apparatus weighed $95 \mathrm{gm}$. (<3\% of the body weight of the eagles). Golden eagles were captured with padded leg-hold traps (after Bloom 1987). All of the golden eagles fitted with telemetry equipment were trapped in the upper Lemhi River Valley. The transmitter numbers were used to identify individual eagles throughout the rest of this paper.

Once captured, the eagles were banded, measured (footpad, wing chord, tarsus and cranium) and photographed. The birds were banded with USF\&WS bands on one leg and an alpha-numeric colored band (orange or green) on the other. The $5 \mathrm{cc}$ blood samples were collected by brachial vein puncture and then were placed in heparinized glass tubes and frozen at the end of each day.

All blood samples were analyzed by Lockheed Martin, a Lockheed Idaho Technologies Company in Idaho Falls, Idaho. They determined lead levels with a VG Elemental PlasmaQuad through inductively coupled plasma-mass spectroscopy (ICP-MS). A wavelength of $207.97 \mathrm{~nm}$ was used for lead, and an internal standard of indium was used at a wavelength of $114.9 \mathrm{~nm}$. The lower limit of reportable residues was $0.01 \mathrm{ppm}$ (wet weight) for lead in all samples. 


\section{TELEMETRY VIA SATELLITE, DATA INTERPRETATION}

In order to maximize battery life of the PTT, transmissions were calibrated for 4 programmed "seasons" and transmission cycles:

30 December 1995 - March 311996

1 April - 30 April 1996

1 May - 30 September 1996

1 October - end of transmissions

$8 \mathrm{hrs}$. on 1 day out of 3

$8 \mathrm{hrs}$. On 3 days out of 7

$8 \mathrm{hrs}$. On 1 day out of 7

$8 \mathrm{hrs}$. on 3 days out of 7

All transmission receptions were processed by Service Argos, Inc. (Landover, MD). Argos categorizes estimates of the PTT positions in location classes (LC) according to their estimated accuracy (Table 1). Accuracy is dependent upon such variables as the number of messages utilized to calculate PTT position and frequency. Each transmitter contains sensors that monitor temperature, battery voltage, and the activity of the transmitter. This information aids in the interpretation of locations of classes, including $0, A, B, C$, or Z. Isolated occurrences of LC's A or lower were not considered reliable data points and so we used them only if they occurred repeatedly in the same area. Our maps of locations of individual birds show LC 3-0 as solid points and all other location classes as open points. Small, round, solid dots on the maps indicate the locations of towns and cities.

Table 1. Description of classification and reliability of satellite location data (Service Argos, Inc., Landover, MD).

\begin{tabular}{|c|c|c|}
\hline LOCATION CLASS & RESOLUTION & \# OF SATELLITES \\
\hline 3 & $\begin{array}{l}68 \% \text { of all points within } 150 \\
\text { meters }\end{array}$ & 4 \\
\hline 2 & $\begin{array}{l}68 \% \text { of all points within } 350 \\
\text { meters }\end{array}$ & 4 \\
\hline 1 & $\begin{array}{l}68 \% \text { of all points within } 1 \\
\mathrm{~km}\end{array}$ & 4 \\
\hline 0 & $1000 \mathrm{~m}+$ & $\begin{array}{l}2 \text { with no auxiliary location } \\
\text { processing }\end{array}$ \\
\hline A & - & $\begin{array}{l}3+\text { auxiliary location } \\
\text { processing }\end{array}$ \\
\hline B & - & $\begin{array}{l}2+\text { auxiliary location } \\
\text { processing }\end{array}$ \\
\hline C & - & $\begin{array}{l}2+\text { auxiliary location } \\
\text { processing }\end{array}$ \\
\hline$z$ & - & not enough \\
\hline
\end{tabular}


Maps and interpretation of data obtained via the satellite system were produced using Ranges V (Kenward and Hodder 1995) and Arcview software (ESRI). Year round range areas were analyzed using convex polygons with number of Incremental Cluster Polygons (groups of locations that separate outliers) identified within the range outline (Hodder et al. 1998).

Seasonal data reported on maps were as follows (based on unpublished migration data from the Snake River Birds of Prey Area, L. Schueck, pers. comm. and Brodeur et al. 1996):

winter - December 1 through March 15;

spring migratory period - March 16 through April 15;

breeding season - April 16 through September 15 and

fall migratory period - September 16 through November 30

We examined the duty cycles from each eagle to determine if movements among individual eagles could be compared. The location estimates from the system were in very similar patterns for the same periods (Appendix A). Therefore, we are confident that similar types of data were collected for each bird and that it is valid to make comparisons among them.

\section{RESULTS}

Two PPTs failed within four months after being placed on the eagles. Tracking of one other eagle continued through the spring and fall migration periods of 1996 and on the three remaining eagles through the following winter. Less than $25 \%$ of the location estimates were categorized as LCs 3-1 (range: $8.8 \%-24.5 \%$; Figure 1) for the eagles monitored.

All the eagles we tracked via satellite had elevated lead levels in their blood (Table 2). Five of these birds were in the lowest category of exposure to lead $(0.20-0.60 \mathrm{ppm})$ and 5727 , an adult male, was in the next higher category $(0.61-1.20 \mathrm{ppm})$, or clinically affected (Kramer and Redig 1997).

Eagle number 5709 was the only adult female golden eagle tracked during the study. Her winter range was $150 \mathrm{~km}$ wide and included both the Lemhi and Pahsimeroi Valleys and adjacent areas to the south and east (Figure 2). However, 5709 did not use the area uniformly, but concentrated her activity in much smaller areas within the winter range.

On 31 March 1996, 5709 began moving northward along the western edge of the Continental Divide. She continued north through the panhandle of Idaho to an area just south of Prince George, British Columbia (Figure 3) where she remained for three days. By April $7^{\text {th }}$, after migrating over $1700 \mathrm{~km}$ in 7 days, the PTT stopped 
transmitting. Therefore, we do not know if the eagle migrated farther north or remained to nest in central British Columbia. This was the only PTT marked bird that migrated out of the eastern Idaho-western Montana area.

Table 2. Summary of telemetry transmission data, blood lead levels, sex and age of 6 golden eagles captured in east central Idaho and tracked by satellite from winter 199596 through winter 1996-97.

\begin{tabular}{cccccccc}
\hline TRANSMITTER \# & AGE & SEX & $\begin{array}{c}\text { PB } \\
\text { LEVEL } \\
\text { (ppm) }\end{array}$ & $\begin{array}{c}\text { DATES OF } \\
\text { TRANSMISSION }\end{array}$ & $\begin{array}{c}\text { MAXIMUM } \\
\text { WINTER AND } \\
\text { SUMMER } \\
\text { RANGE } \\
\text { WIDTH IN } \\
\text { KM }^{1}\end{array}$ & $\begin{array}{c}\text { TOTAL \# } \\
\text { FIXES }\end{array}$ & $\begin{array}{c}\text { \% FIXES } \\
\text { LC } 1-3\end{array}$ \\
\hline 5709 & A & F & 0.23 & $1 / 13 / 96-4 / 7 / 96$ & 150 & 157 & $14.6 \%$ \\
5727 & A & M & 0.71 & $1 / 18 / 96-1 / 2 / 97$ & 350 & 271 & $15.5 \%$ \\
5726 & A & M & 0.42 & $12 / 30 / 95-1 / 2 / 97$ & 80 & 322 & $16.1 \%$ \\
5699 & SA & M & 0.22 & $1 / 18 / 96-5 / 13 / 96$ & 150 & 192 & $24.5 \%$ \\
5738 & SA & M & 0.37 & $1 / 15 / 96-1 / 3 / 97$ & 200 & 238 & $8.8 \%$ \\
5707 & SA & F & 0.39 & $1 / 8 / 96-11 / 26 / 97$ & 160 & 252 & $16.7 \%$ \\
\hline
\end{tabular}

${ }^{1}$ Winter and summer rainge width is approximate and measured at the greatest distance between outermost points. It does not include distances covered during migration.

We captured and radio-tagged two adult male eagles. Male 5727, had the highest lead level of the 6 eagles tracked by satellite. He wintered in two distinct, but widely separated locations ( $350 \mathrm{~km}$ apart) in Idaho (Figure 4). One of these locations included the Lemhi and the Pahsimeroi Valleys, although the eagle spent more time in the Lemhi Valley. The other area was to the southwest, near Twin Falls, Idaho. Each wintering area was about $75 \mathrm{~km}$ in diameter.

During the spring, 5727 flew north $400 \mathrm{~km}$ to the area around Polaris, Montana (Figure 5). The eagle remained in this general area for the first two weeks in April. In late April, he began to drift southward again and all subsequent locations during the nesting season were scattered along the Continental Divide, south and west of Polaris (Figure 6). We assume that 5727 did not breed successfully in 1996 because these locations were widely dispersed. This eagle used a range during the breeding season at least $130 \mathrm{~km}$ wide. Eagle 5727 moved southward in the fall to the Twin Falls area along the same route he had taken in the spring (Figure 7) and spent most of that winter there. 

The other adult male eagle, 5726, was a year-round resident in the Lemhi Valley, although he made several short visits to the Pahsimeroi Valley, as well (Figure 8). He was the only resident eagle we tracked and had a winter range about $80 \mathrm{~km}$ long. During the nesting season and migration periods 5726 occupied an area only $50 \mathrm{~km}$ in diameter (Figures 9, 10,11 and 12). This bird's summer range was entirely within his winter range. Unfortunately, we received the radio location data in mid-summer after young normally fledge from the nest, so we were unable to verify nesting by 5726 in 1996. However, in July we visited the area 5726 frequented most during the nesting season and spotted an adult male golden eagle marked with a backpack transmitter. This eagle, most probably 5726, was flying near a larger adult and an immature golden eagle. All three birds were near a known nesting territory in the upper end of the Lemhi Valley and it is probable that he nested successfully in this area.

We placed PTTs on two subadult males and one subadult female golden eagle. None of these birds were long-range migrants. The location estimates for the subadults were more widely scattered throughout their entire range than for adults. In addition, movement patterns of subadults and breeding adults were different, particularly during the breeding season. With the exception of the migration period, subadult eagles tended to be more wide-ranging, often moving more frequently to different locations and greater distances between satellite locations than adult birds (see Appendix B for movement patterns of individual eagles and Appendix $C$ for monthly maps of location estimates for individual eagles). This difference in movement patterns was less obvious during the winter months.

One subadult male (5699) wintered in both the Lemhi and Pahsimeroi Valleys but also spent time in an adjac *nt valley in Montana (Figure 13). Eagle 5699 had a winter range about $150 \mathrm{~km}$ in width. This eagle's spring and summer movements overlapped his winter range (Figures 14, and 15). The PTT of this subadult bird failed in May. His range from 18 January 1996 through 13 May 1996 spanned approximately $150 \mathrm{hm}$ (Figure 16).

The other subadult male's winter movements (eagle 5738) were also approximately 150 $\mathrm{km}$ at the widest point (Figure 17). His winter range was similar to 5699's, which included the Lemhi Valley and southwestern Montana. However, 5738 was never located in the Pahsimeroi Valley. His spring, summer and fall movements overlapped the part of his winter range in Montana but not the part in Idaho (Figures 18, 19 and 20). The summer locations for 5738 were scattered more widely than those in the winter and consequently his year-round range spanned a distance more than $200 \mathrm{~km}$ in width (Figure 21).

The subadult female we studied (eagle 5707) spent more time in the Pahsimeroi and Upper Little Lost River Valleys than in the Lemhi Valley during the winter (Figure 22). Her winter range spanned about $160 \mathrm{~km}$ and, like the other nonmigratory eagles, her spring, fall and summer range overlapped her winter range (Figures 23,24, 25 and 26). 5707 did not visit the Pahsimeroi Valley during the summer. 
Year-round home range areas of the adult golden eagles monitored were generally smaller than those of subadult eagles (Table 3). Use of only LCs 1-3 underestimated areas used by the birds because sample sizes were small within these categories. With the exception of eagle 5707, it also underestimated the number of concentrated use areas (clusters) within each golden eagle range. The number of clusters (LCs 3-0) within a use area varied from 8 for adult male 5709 , to one for subadult female 5707 . Use of only LCs 3-1 resulted in the identification of 2-3 clusters within each eagle home range.

Clusters identified within the east central Idaho study area during the winter were in areas of native shrub habitats. Winter ranges of most of the eagles overlapped spatially and sometimes temporally, as well. Winter ranges of eagles $5727,5699,5738$ and 5707 were smaller than during the breeding season. Three of these birds were subadults and one was a non-nesting adult. The one resident adult male that we assume nested had a larger winter range than home range during the nesting season. No data were collected from the adult female, 5709 during the breeding season, so no comparison of range sizes could be made for this eagle.

Table 3. Summary of telemetry data (convex polygons) for location classes 3-0, based on data from 6 golden eagles from January 1996 through September 1997.

\begin{tabular}{cccccccc}
\hline TM\# & $\begin{array}{c}\text { Range } \\
\text { (ha) } 90 \% \\
1-0\end{array}$ & $\mathbf{n}$ & $\begin{array}{c}\text { Range } \\
\text { (ha) } 90 \% \\
1-3\end{array}$ & $\mathbf{n}$ & $\begin{array}{c}\text { Maximum } \\
\text { ha } \\
\text { (convex } \\
\text { polygon) }\end{array}$ & $\begin{array}{c}\text { \# clusters } \\
3-1\end{array}$ & $\begin{array}{c}\text { \# clusters } \\
3-0\end{array}$ \\
\hline 5709 & 54930 & 91 & 42,070 & 16 & 764,481 & 3 & 8 \\
5727 & 73,910 & 70 & 38,140 & 24 & 557,148 & 2 & 4 \\
5726 & 66,420 & 109 & 8,623 & 24 & 442,076 & 2 & 2 \\
5699 & 112,800 & 93 & 60,160 & 34 & 415,246 & 2 & 4 \\
5738 & 172,000 & 74 & 26,410 & 12 & 686,907 & 2 & 4 \\
5707 & 561,900 & 82 & 124,800 & 25 & $1,115,259$ & 2 & 1 \\
\hline
\end{tabular}

\section{DISCUSSION}

The golden eagle use areas reported herein are larger than those reported by researchers in Idaho who used conventional telemetry (Marzluff et al. 1997, Dunstan et al. 1978). One possible explanation is that the previous studies have been conducted on eagles during the nesting season, or on resident birds. However, 
location data obtained with the Argos satellite system cannot be interpreted in the same way as conventional telemetry data because so few satellite location estimates fall within the most accurate location classes (3-1). Generally, there is less error in conventional telemetry location data. Schueck et al. (1994) also reported golden eagle winter use areas determined by PTT location estimates to be larger, in most cases, than use areas determined by direct observations obtained in conjunction with conventional telemetry.

In spite of the error associated with PTT location estimates, the data are valuable for certain interpretations, since they can be used to infer general use areas and movements of individual eagles throughout the tracking period. In addition, these data are particularly useful for relative comparisons of use areas, movement patterns among eagles, and for monitoring individuals over large areas and for extended periods. For instance, we found year-round use areas of subadult golden eagles generally to be larger than those of adults, and subadults and nonbreeding adults to be somewhat nomadic. Schueck et al. (1994) reported similar results on eagles studied in the Snake River Birds of Prey area. We also noted that non-breeding season use areas did not necessarily overlap the home range used during the breeding season.

Golden eagles in the study area showed fidelity to wintering areas from one year to the next and although overall use areas were large, activity was generally concentrated in small areas of native shrub habitat at lower elevations. In fact, the most successful winter trapping areas were those sagebrush areas in which concentrations of blacktailed jackrabbits occurred. Previous research has reported similar habitat selection by wintering eagles, as well as, for resident birds (Marzluff et al. 1997, Craig et al. 1986). In addition, our data, as well as those collected by Marzluff et al. (1997), show that not only do eagles use the same areas from year-to-year, but that size and shape of those areas are often similar among years.

Although we have observed adult eagles (we assumed resident birds) to occasionally display undulating flights during the winter, wintering areas of the eagles overlapped and we trapped up to 8 eagles in a single day within the same general area. This implies that there is no territorial defense during the winter. Others have reported overlapping use areas during the nonbreeding season (Marzluff et al. 1997, Schueck et al. 1994).

Argos telemetry data for the 6 eagles studied indicated that the wintering population of eagles in the study area comprises local resident birds, long-distance migrants and regional residents that range over large portions of eastern Idaho and western Montana. The only area used in common by all of the eagles was the Lemhi Valley during the winter.

The Pahsimeroi and Lemhi Valleys were included in the winter ranges of five of the 6 golden eagles tracked during winter 1995-96. However, only two birds, 5699 and 5707 (both subadults), were located frequently in both valleys. These findings are supported 
by our recapture data that indicate a limited exchange of eagles between the two valleys. Few PTT location estimates from any of the 6 eagles were from the Birch Creek Valley.

Golden eagles show fidelity to wintering and nesting areas and also follow the same migration routes from one year to the next (Marzluff et al. 1997, Brodeur et al. 1996, Craig and Craig 1996, L. Schueck 1994). If this is true for the 6 golden eagles that we studied, the Argos telemetry data collected in 1995-1996 can be used to infer the areas frequented by these birds prior to their capture. Therefore, we assume that the elevation in blood lead levels found in all 6 of the eagles, probably resulted from exposure to lead within the annual ranges we have documented.

Therefore, adult male 5726 , a year-round resident of the study area, was most likely exposed to lead within the Upper Lemhi Valley. Similarly, the four eagles that were "regional residents" probably were exposed to lead either in, or within several hundred kilometers, of our study area. The single long-range migrant may have been exposed to lead on its winter range in our study area in Idaho, on its summer range, or along its migration route into Canada.

\section{MANAGEMENT IMPLICATIONS}

As a result of this research we have determined that:

- the study area is used regularly by wintering golden and bald eagles;

- the wintering golden eagle population comprises resident nesters, regional residents, and migrants;

- the bald eagles in the study area are primarily winter residents with only three known pairs of bald eagles resident in the study area;

- many of the bald and golden eagles wintering in east central Idaho have elevated lead levels in their blood;

- wintering bald eagles in the study area have elevated mercury levels;

- contaminant levels in most of the eagles sampled are sublethal;

- exposure to lead contaminants may be chronic;

- the specific effects of sublethal, chronic exposure of lead to individual eagles, as well as the eagle population at large is unknown;

- specific sources and pathways of lead contamination are yet to be determined;

- live prey within wintering or natal ranges of golden eagles are probably not the avenue of lead contamination to wild golden eagles;

- lead contamination is acquired locally/regionally by some eagles;

- lead contamination of golden eagles in western North America is probably a pandemic phenomenon. 


\section{MANAGEMENT RECOMMENDATIONS}

1. Continue to monitor blood lead levels in wintering golden eagles to provide further insight into long-term trends and threats to golden eagle populations. Recapture data are particularly important because they allow the tracking of individual eagles through time.

2. Fund research to monitor the movements and fates of individual golden eagles with and without elevated lead levels over long periods of time (reproduction, mortality, etc.) via satellite telemetry. Such long-term data are the key to determining the affects of sub-lethal lead contamination on eagle populations, as well as, the geographic source of the lead.

3. Fund research to conduct DNA analysis on blood samples currently being stored from this study. We have samples from nestling eagles in our study area, an eagle from Alaska that wintered in our study area, the eagles whose movements we followed via satellite and samples from eagles from the Snake River Birds of Prey Area. DNA analysis of these samples would help determine the genetic relationships of the birds wintering in east central Idaho and possibly, the areas from which they originated. These data could be compared to data already collected in this study, and used to determine if there is a correlation between related birds and contaminant levels (e.g., do more eagles that are regional residents have elevated lead levels than eagles from Alaska?).

4. Eagles rely on the native shrub habitats where jackrabbits, an important prey base, occur. Management of golden eagle populations should include protection of these areas.

5. Researchers have recently reported downward trends in golden eagle populations. It is important to monitor golden eagle populations to determine if this trend continues.

\section{ACKNOWLEDGMENTS}

We gratefully acknowledge Allan Thomas for his ongoing support of this project. Mark Fuller provided invaluable assistance for the radio-tracking portion of the study. Wayne Melquist, Mike Scott, Tom Parker, Sally Gregory and Robin Holmquist have all helped at various times. Linda Schueck provided maps of eagle locations and assistance in interpreting location estimate data. Fred Martinson provided statistical advice and did the statistical analyses. Christian Klaus produced maps in earlier 
versions of this paper. Al Harmata offered advice on trapping eagles in the beginning stages of this research. We also wish to thank the following people who assisted with fieldwork: J. and B. O'Bannon, M. and D. Kossler, J. and J. Amonson, K. and J. Slagg, S. Horton, and Salmon District BIM personnel, Loren Anderson and Helen Ulmschneider. In addition, we thank the owners of the Amonson, Shiner, Mckinney and Kruckeberg ranches for allowing us to trap eagles on their property. Special thanks go to Lockheed Martin, a Lockheed Idaho Technologies Company in Idaho Falls, Idaho for generously donating the support of their staff and equipment for analysis of $\mathrm{Pb}$ levels in 48 golden eagle bloods in 1996.

This research has been funded by: the U.S. Bureau of Land Management; The U. S. Geological Survey Biological Resources Division, Snake River Field Station; the Idaho Department of Fish and Game; Western Ecological Studies Team; the Boise Chapter of the Audubon Society; and the Idaho Wildlife Society. 


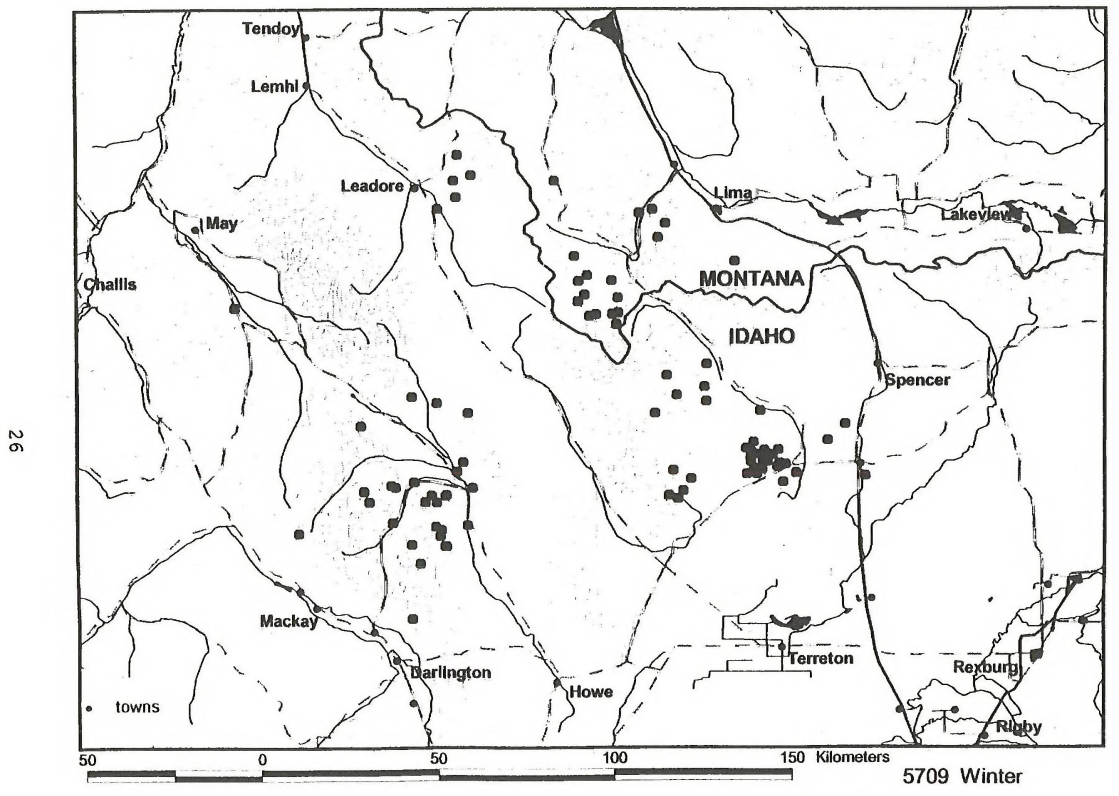

Figure 2. Range of an adult female golden eagle (transmitter 5709) in the Intermountain West during winter 1995-96. 


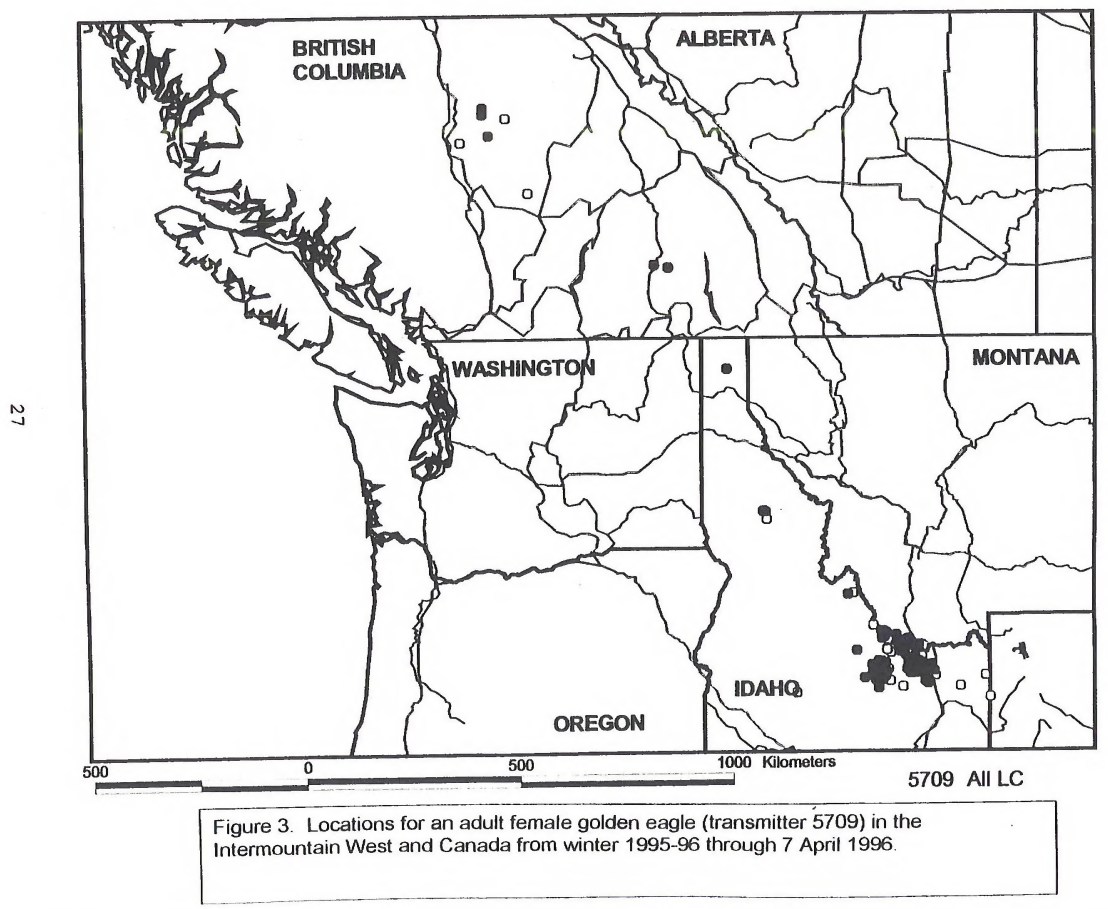




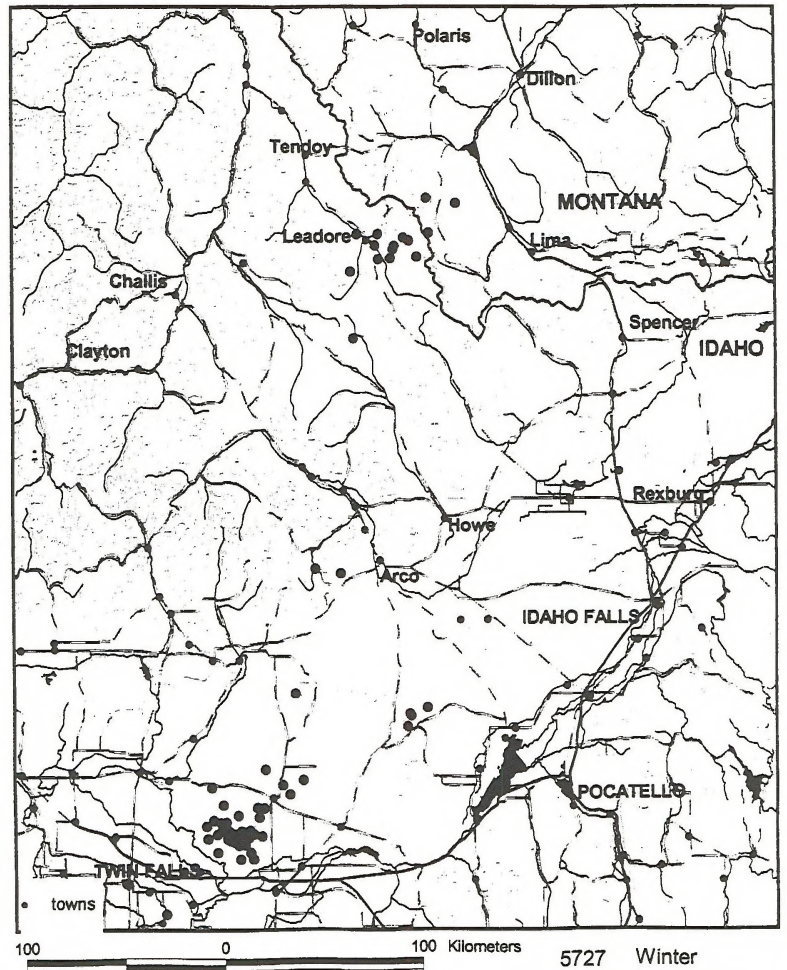

Figure 4. Range of an adult male golden eagle (transmitter 5727) in the Intermountain West during winter 1995-96. 


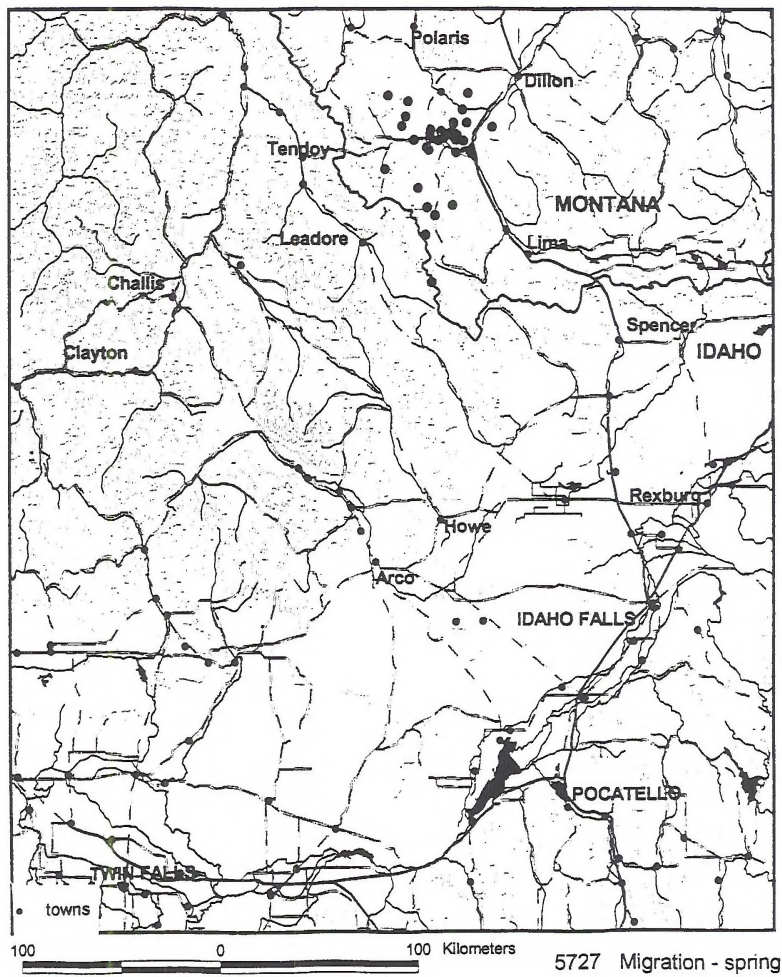

Figure 5. Locations for an adult male golden eagle (transmitter 5727) in the Intermountain West during the spring 1996 migration period. 


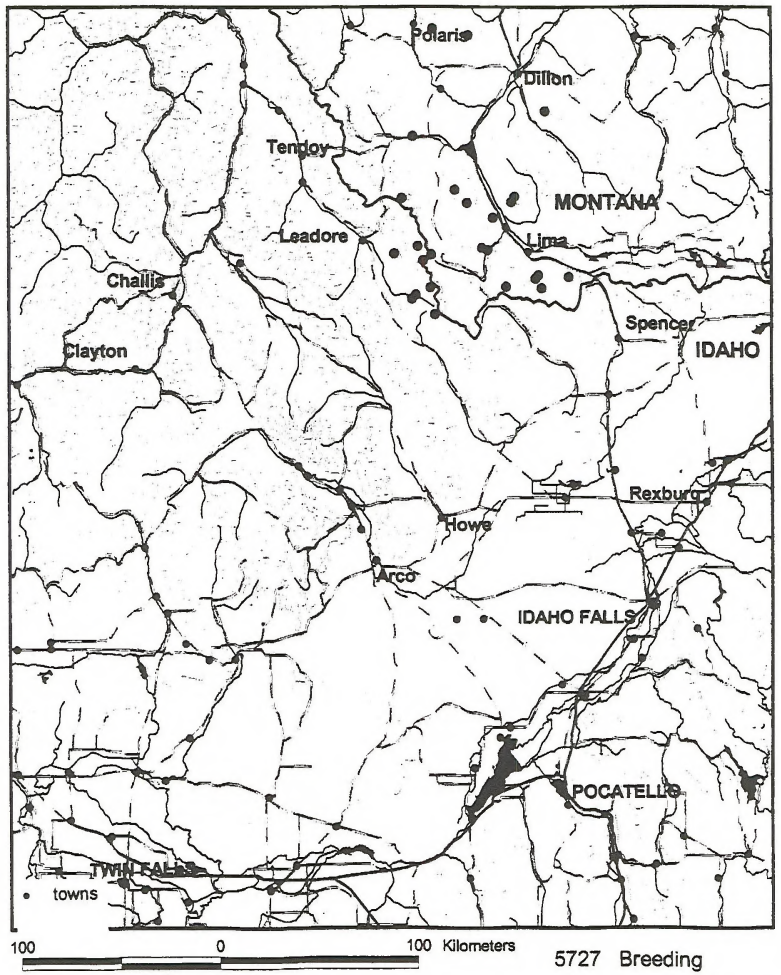

Figure 6. Home range of an adult male golden eagle (transmitter 5727) in the Intermountain West during breeding season 1996. 


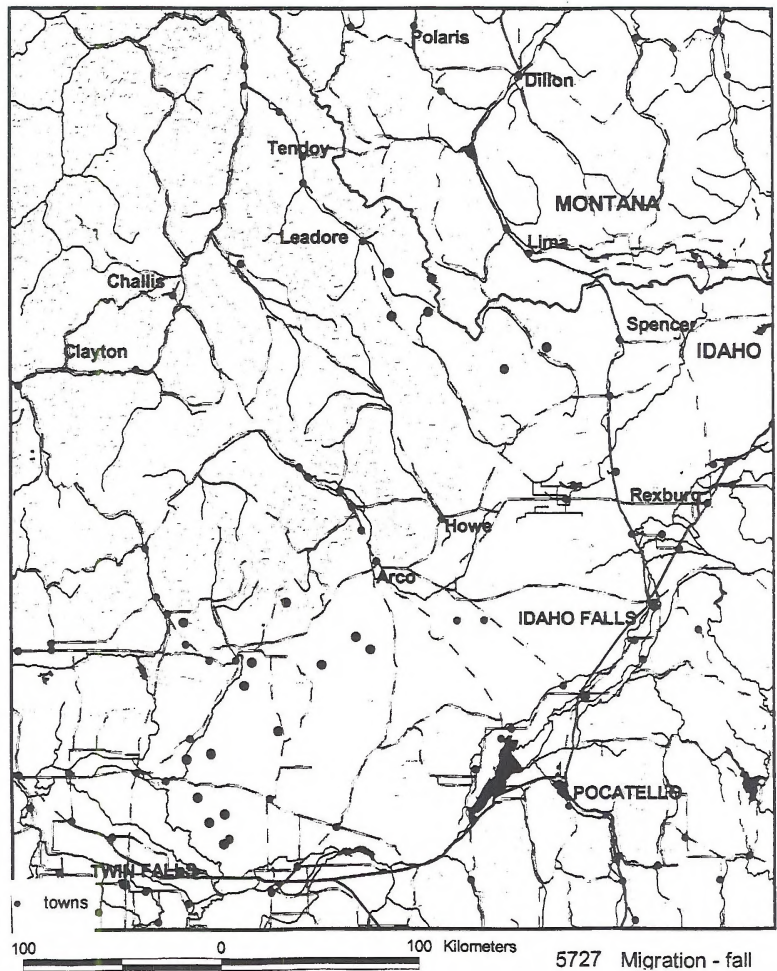

Figure 7. Locations for an adult male golden eagle (transmitter 5727 ) in the Intermountain West during fall migration 1996. 


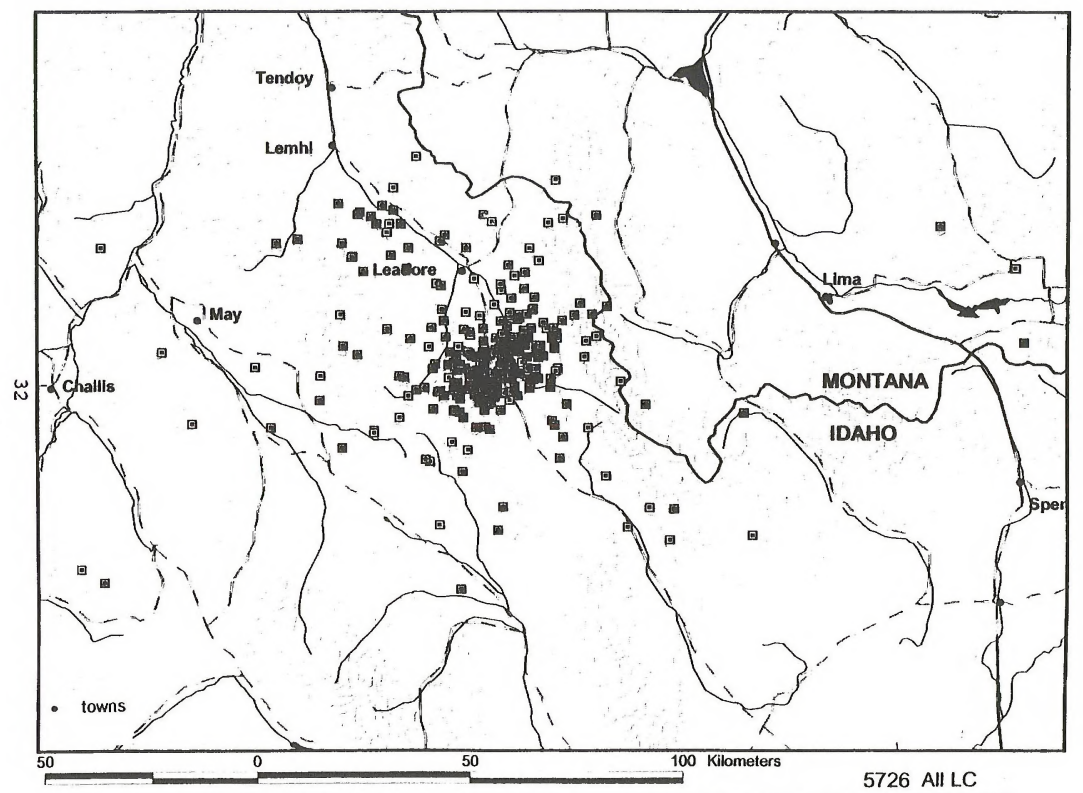

Figure 8. All locations for an adult male golden eagle (transmitter 5726) in east central Idaho from 30 December 1995 through 2 January 1997. 


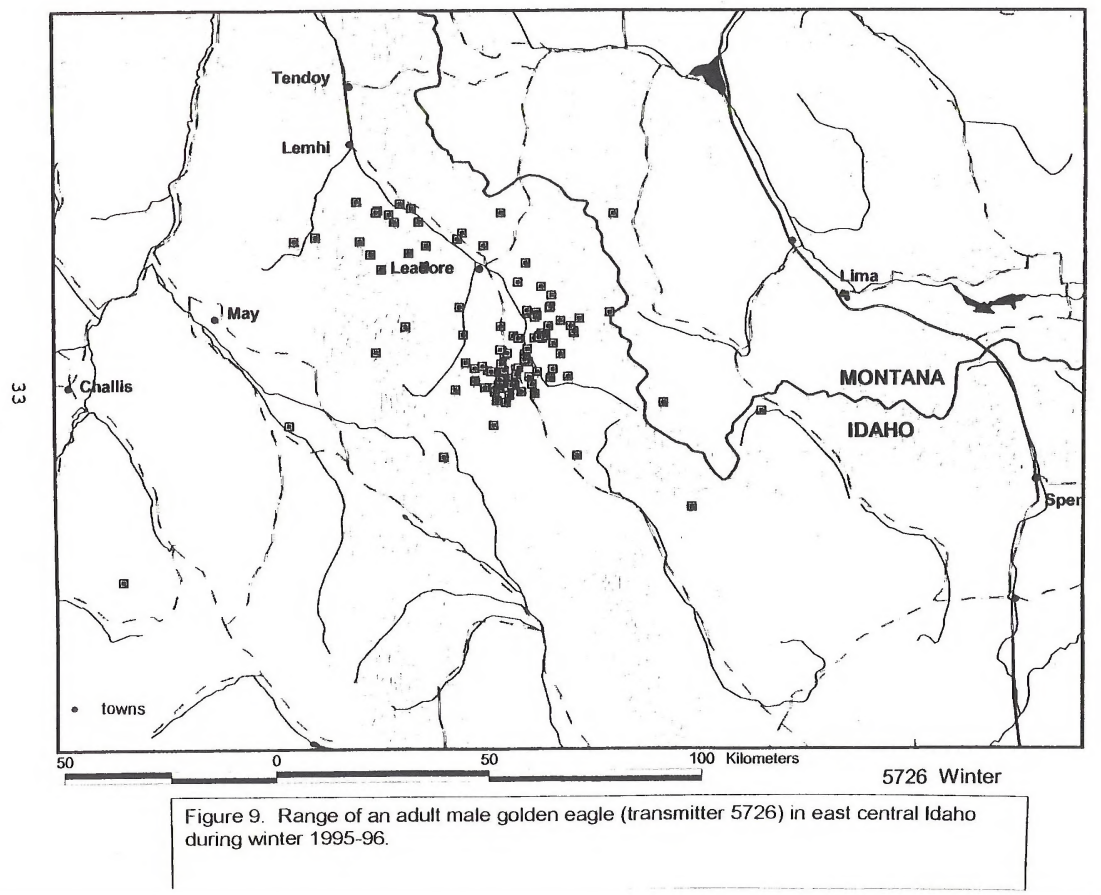




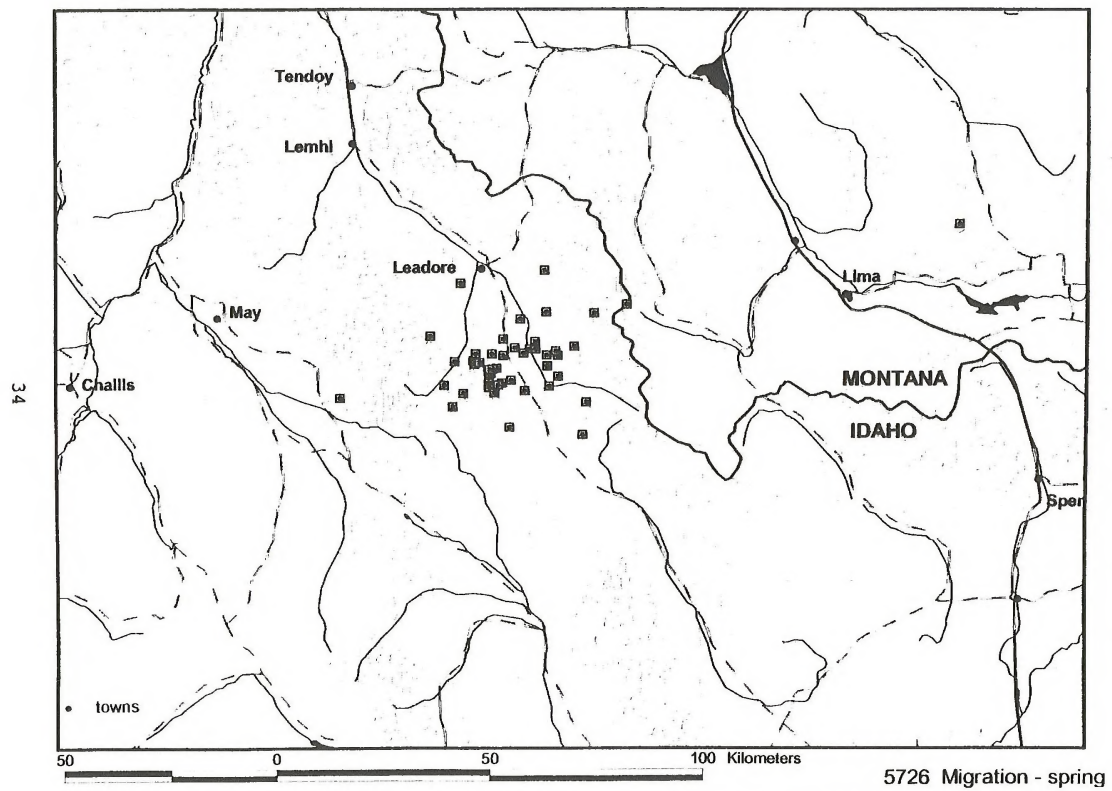

Figure 10. Locations for an adult male golden eagle (transmitter 5726) in east central Idaho during spring migration 1996. 


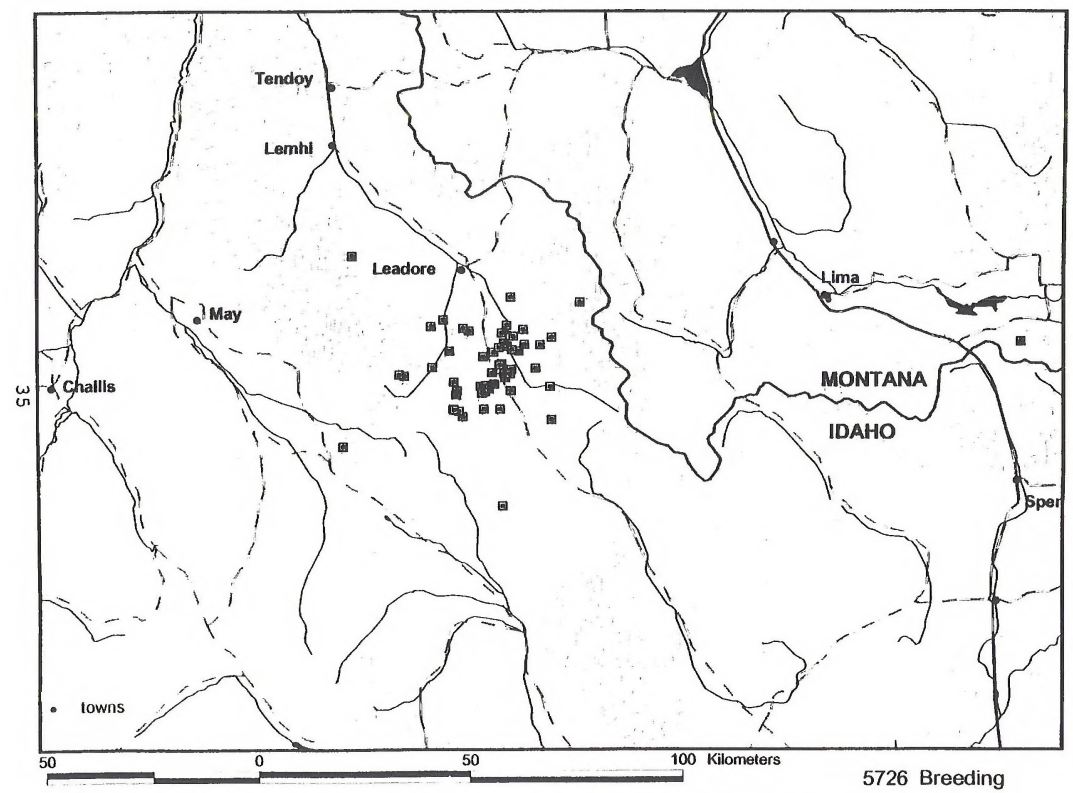

Figure 11. Home range of an adult male golden eagle (transmitter 5726) in east central Idaho during breeding season 1996. 


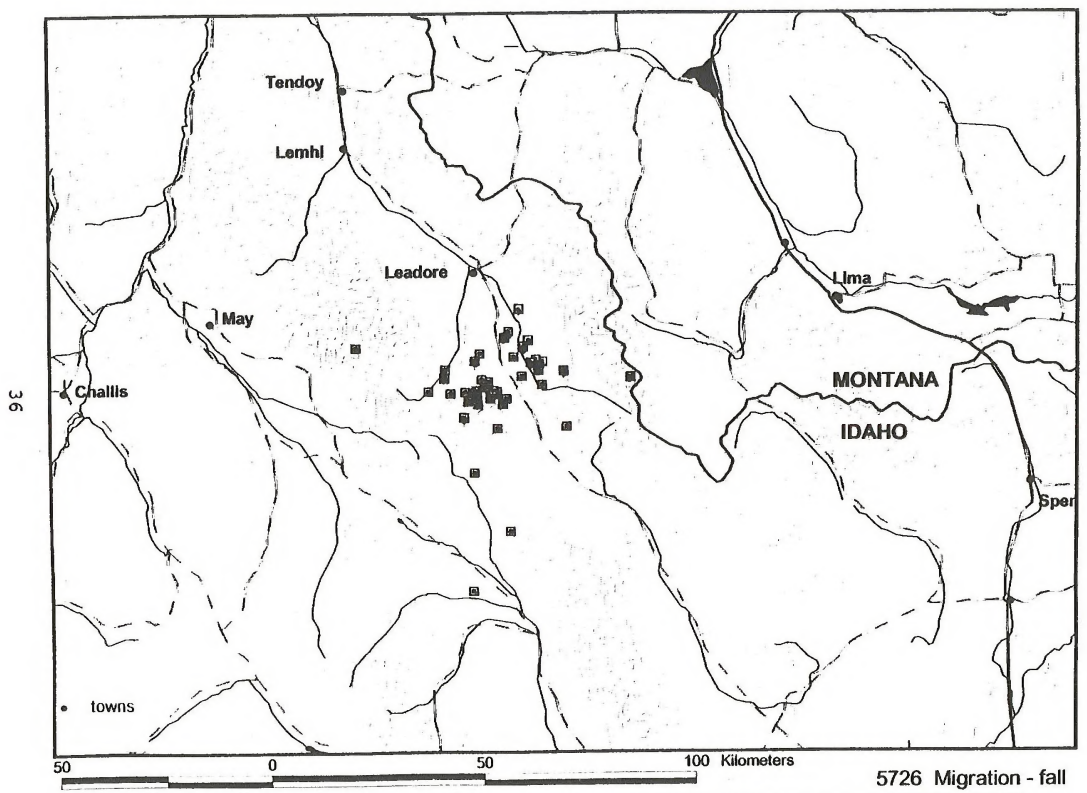

Figure 12. Locations for an adult male golden eagle (transmitter 5726) in east central Idaho during fall migration 1996. 


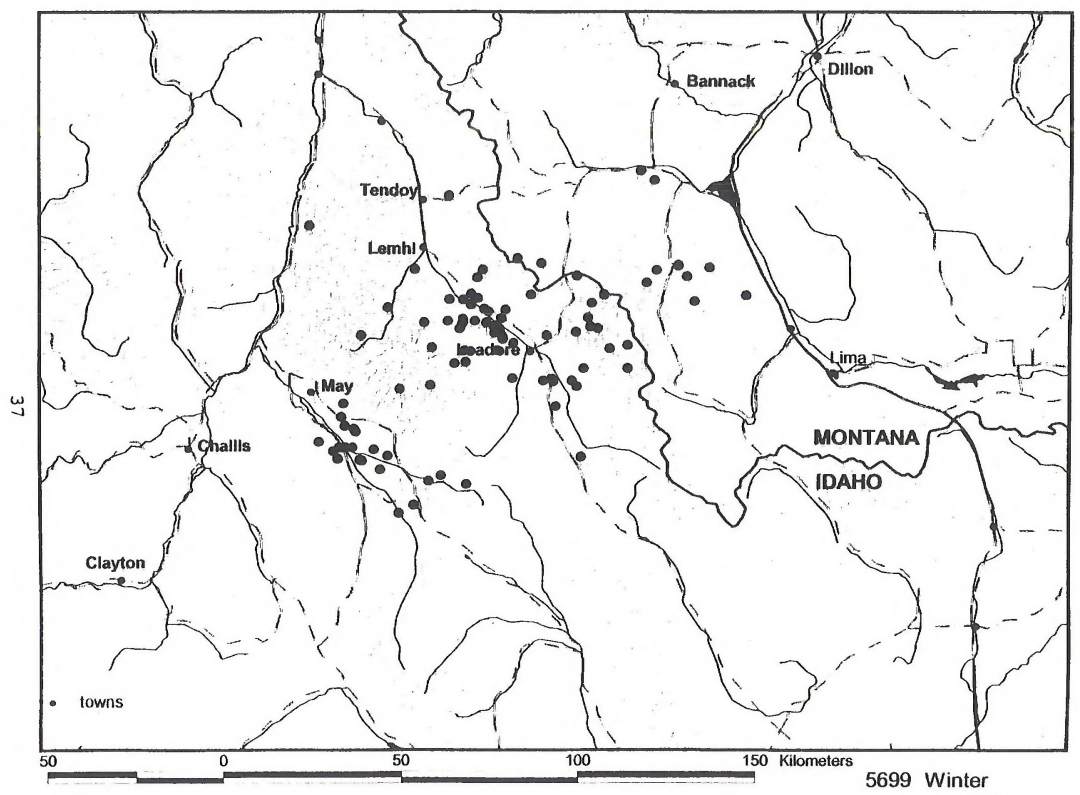

Figure 13. Range of a subadult male golden eagle (transmitter 5699) in the Intermountain West during winter 1995-96. 


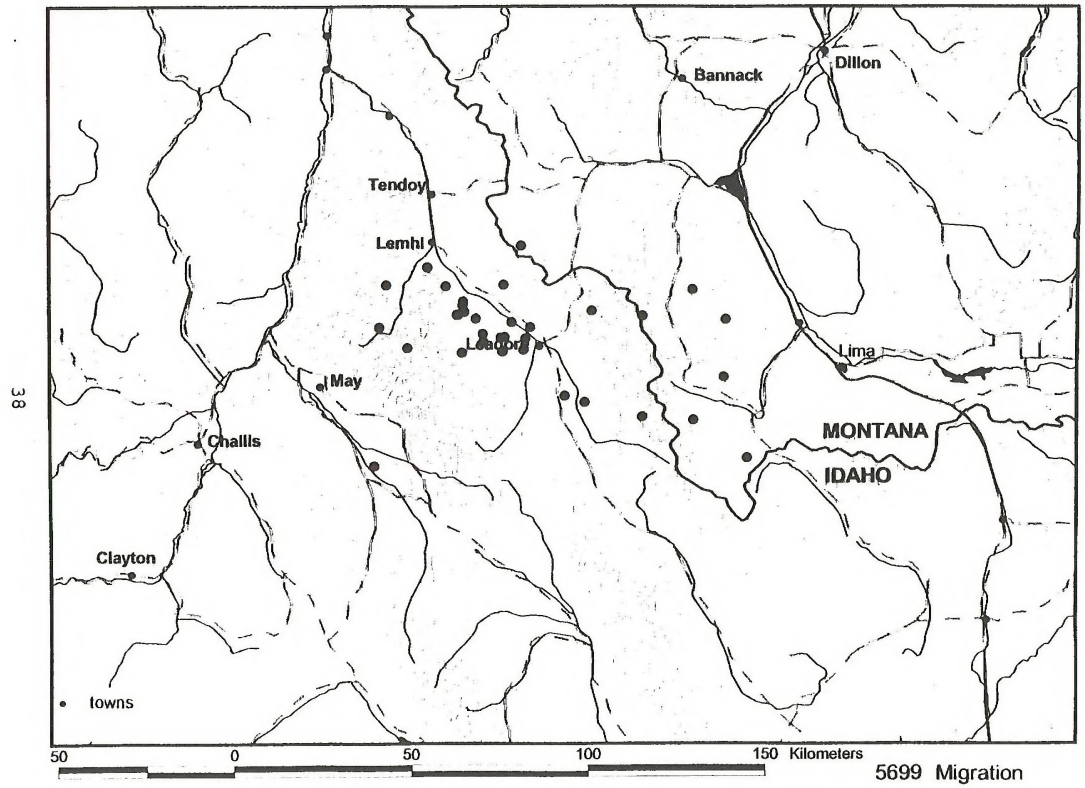

Figure 14. Locations for a subadult male golden eagle (transmitter 5699) in the Intermountain West during spring migration 1996 


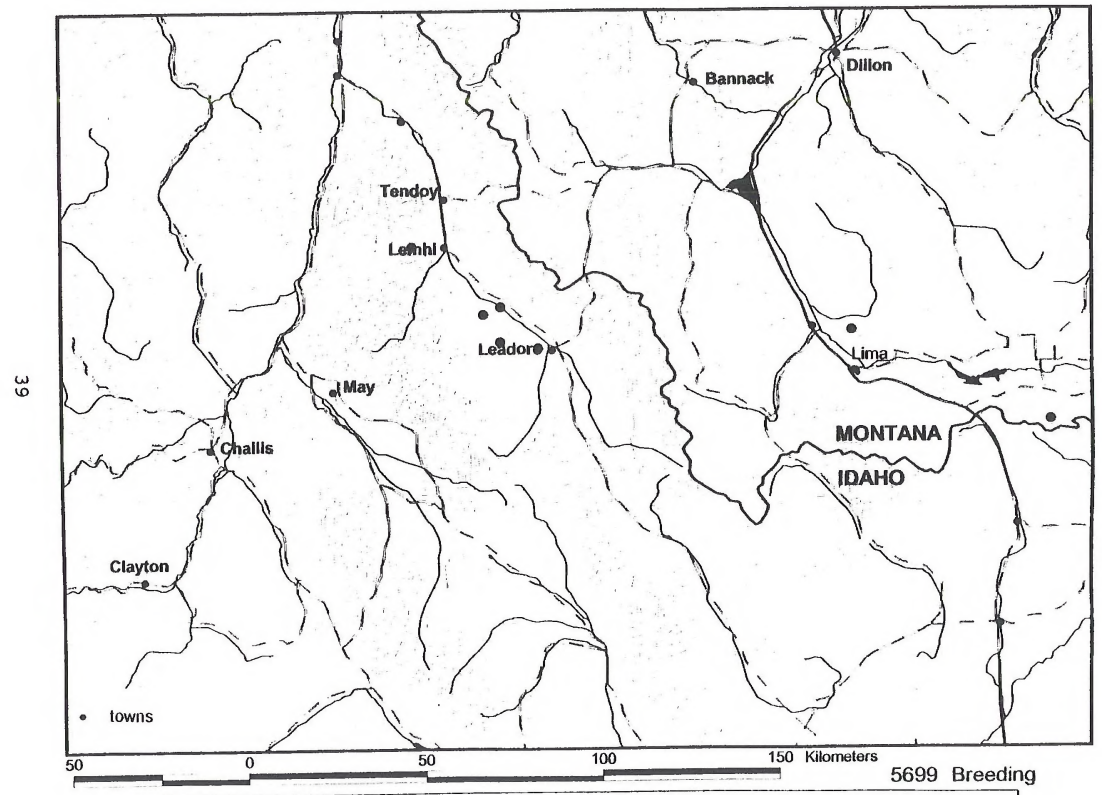

Figure 15. Home range of a subadult male golden eagle (transmitter 5699) in the Intermountain West during breeding season 1996 


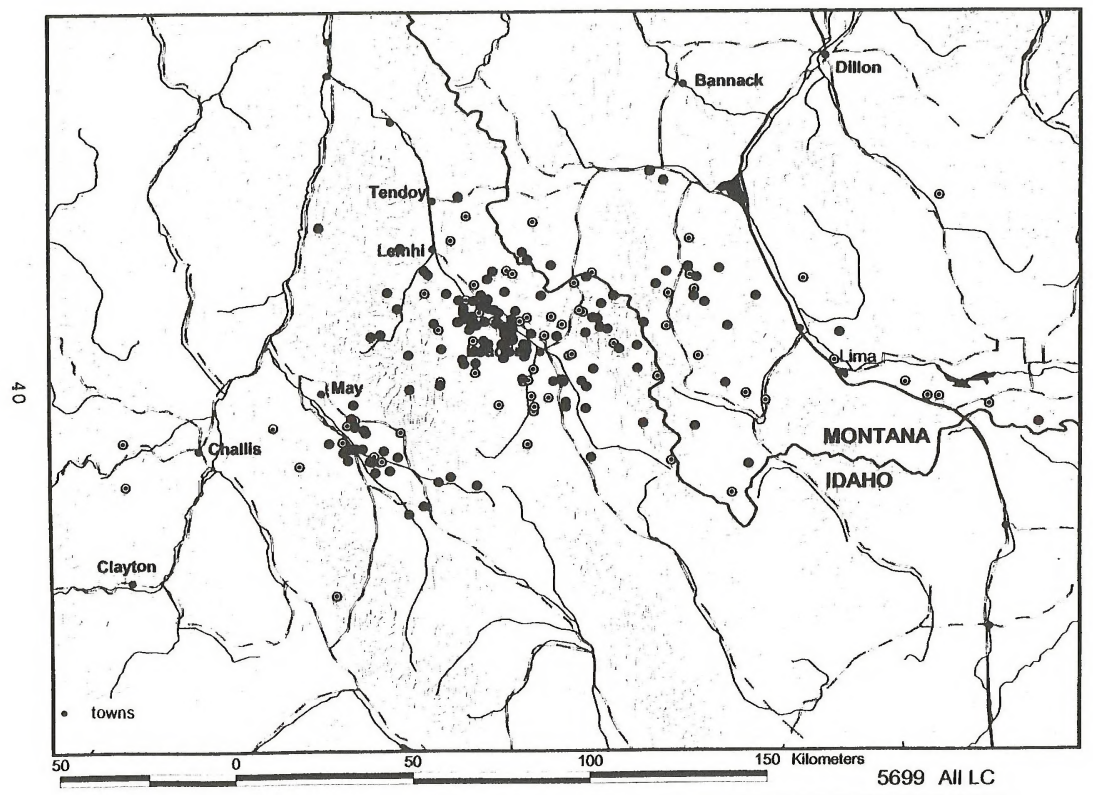

Figure 16. All locations for a subadult male golden eagle (transmitter 5699) in the Intermountain West from 18 January 1996 through 13 May 1996. 


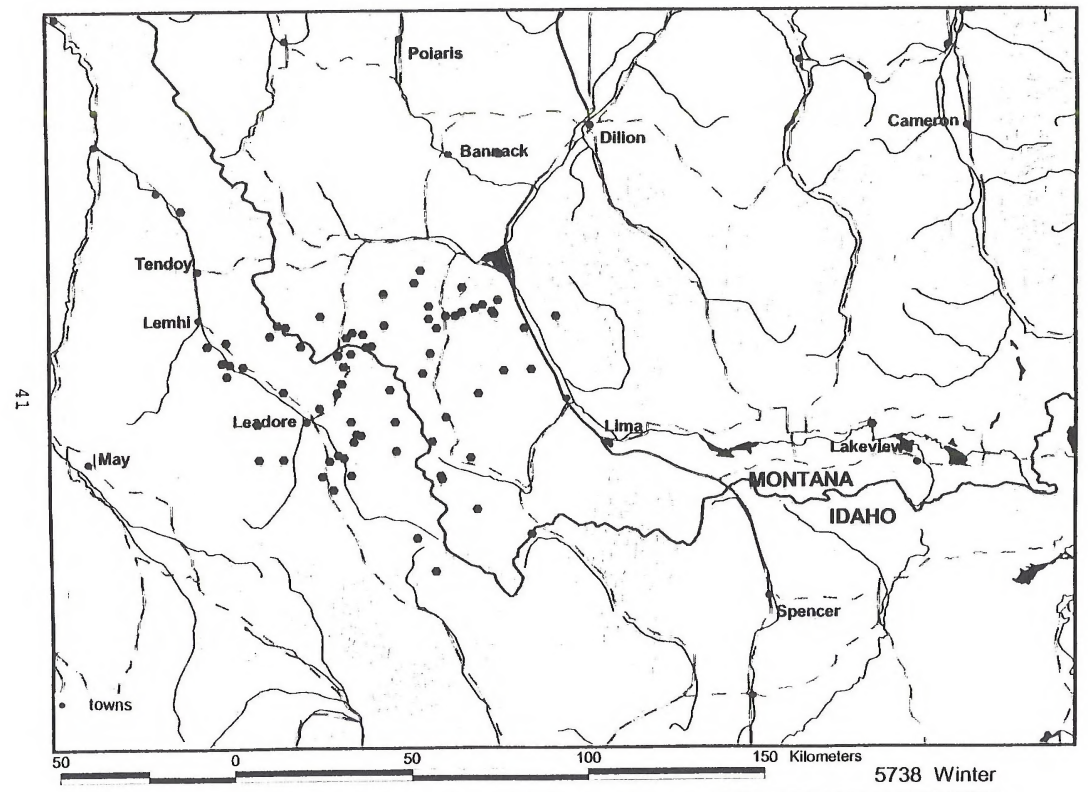

Figure 17. Range of a subadult male golden eagle (transmitter 5738 ) in the Intermountain West during winter 1995-96. 


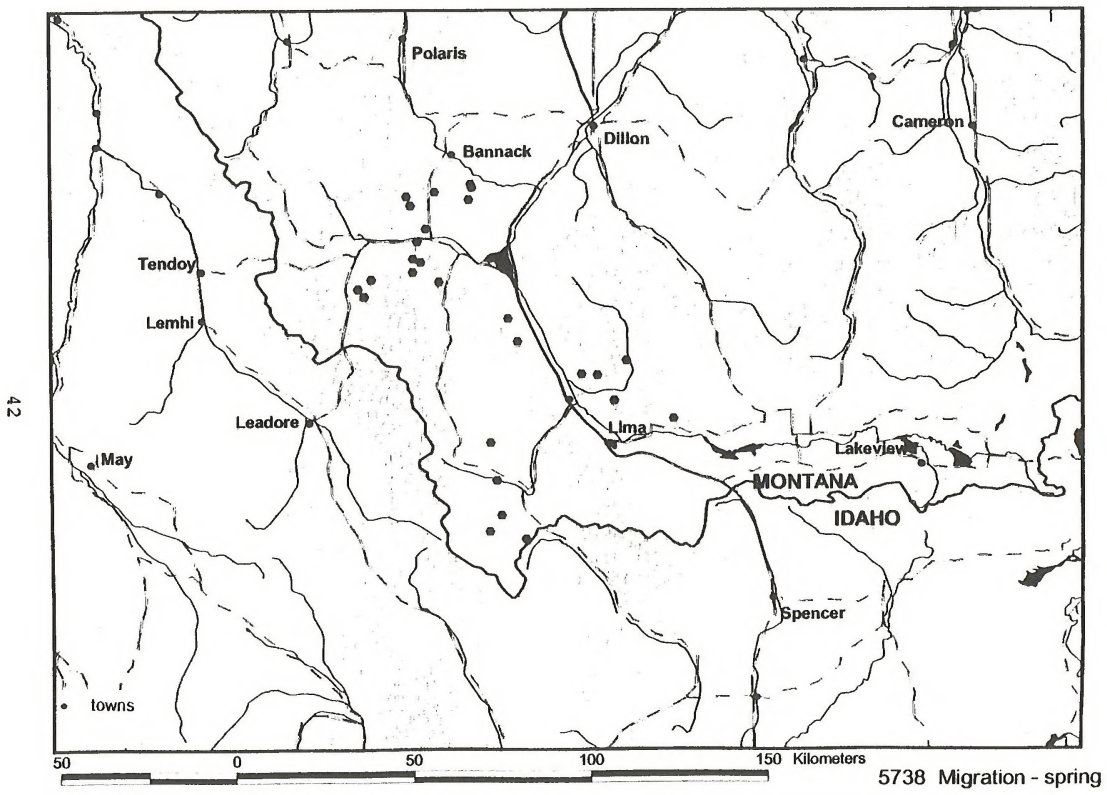

Figure 18. Locations for a subadult male golden eagle (transmitter 5738 ) in the Intermountain West during spring migration 1996. 


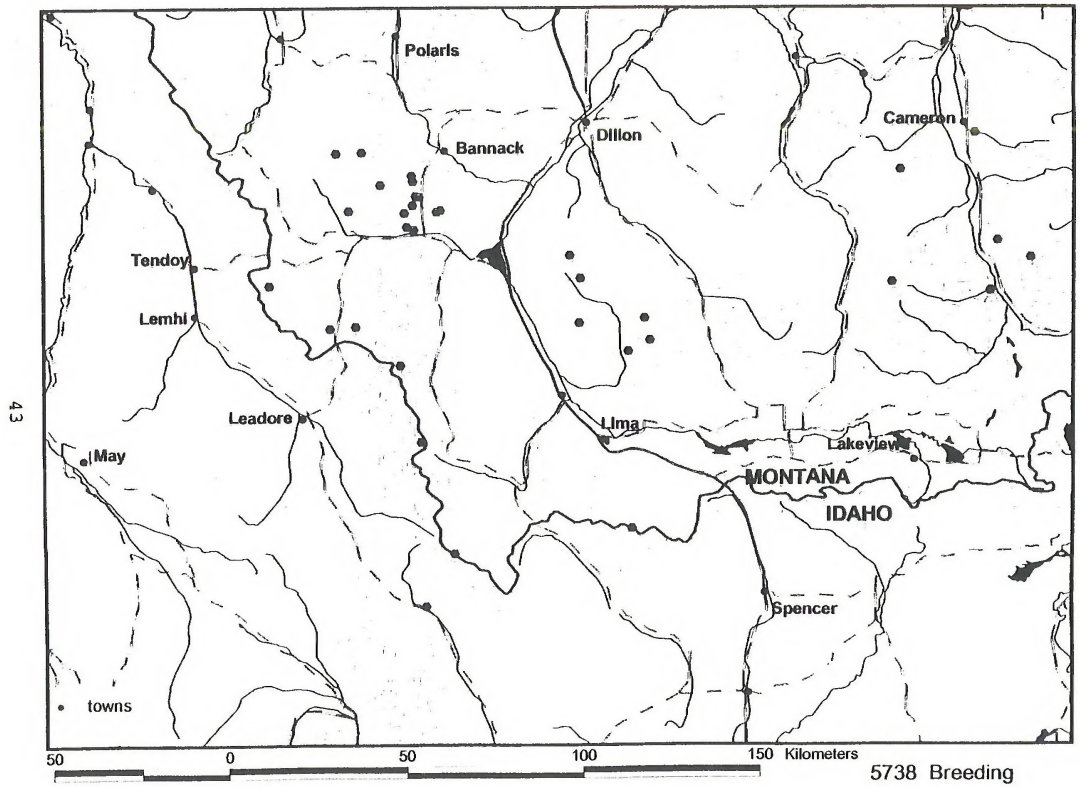

Figure 19. Locations for a subadult male golden eagle (transmitter 5738 ) in the Intermountain West during breeding season 1996. 


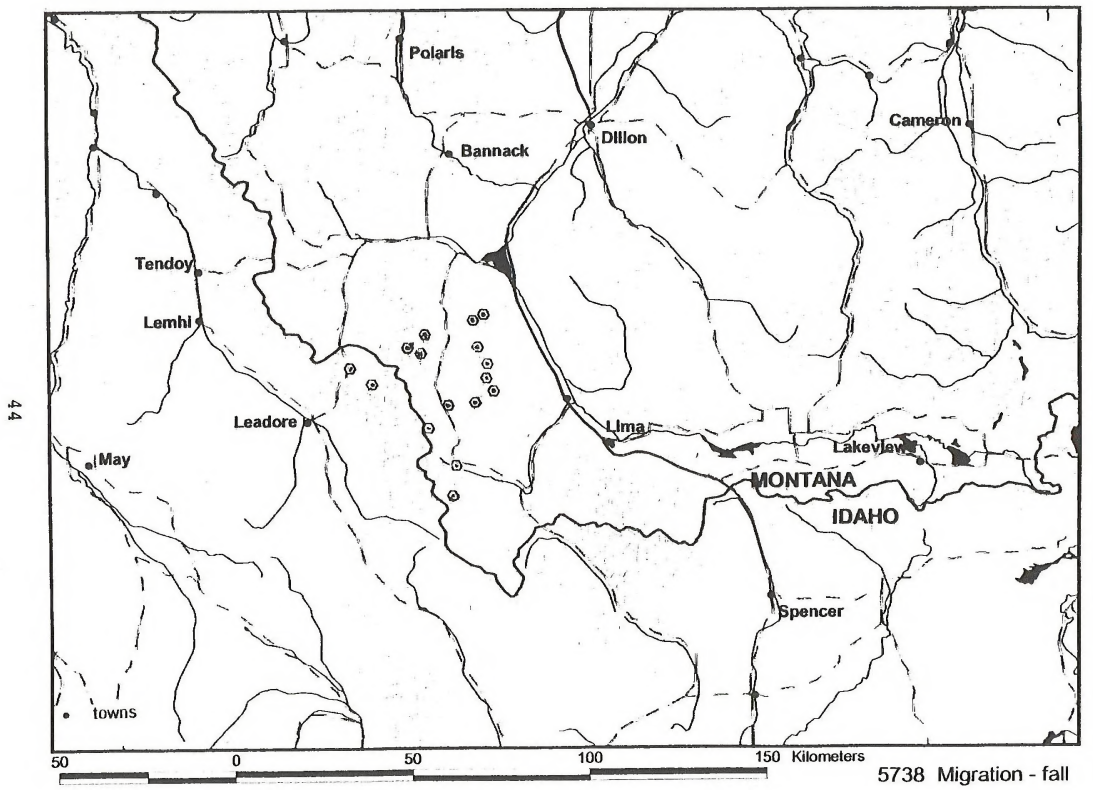

Figure 20. Locations for a subadult male golden eagle (transmitter 5738 ) in the Intermountain West during fall migration 1996. 


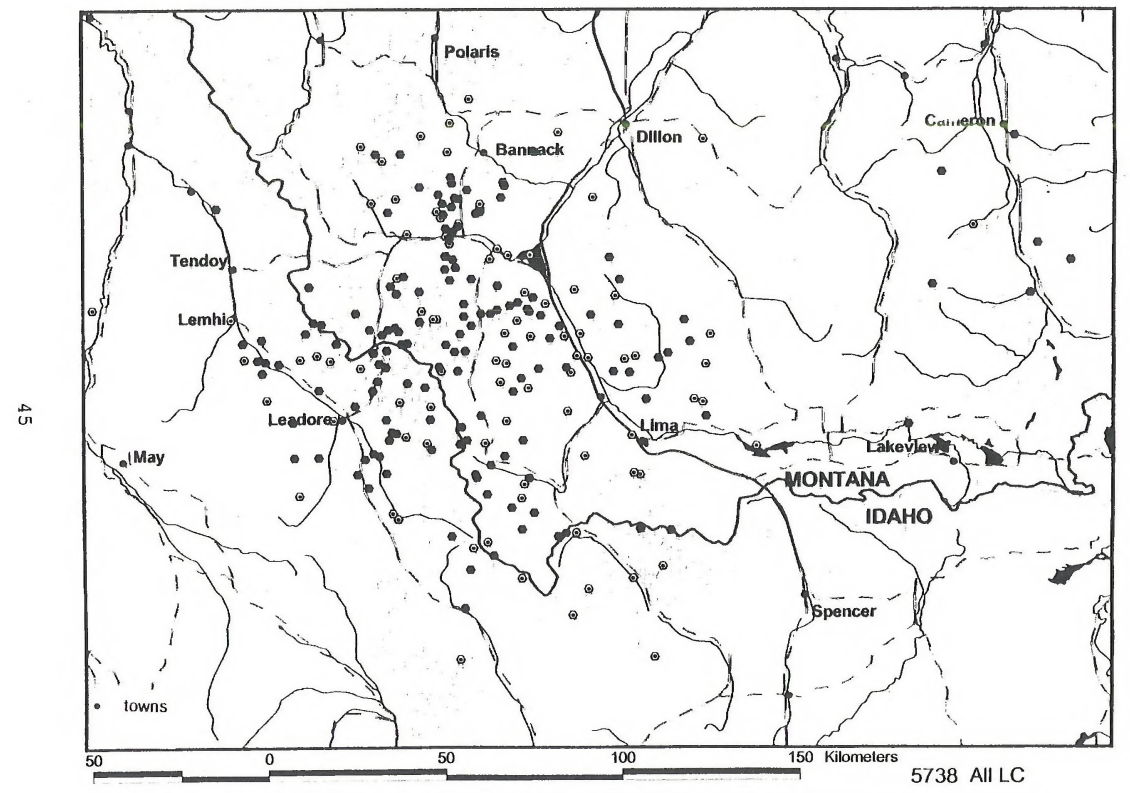

Figure 21. All locations for a subadult male golden eagle (transmitter 5738) in the Intermountain West from 15 January 1996 through 3 January 1997. 


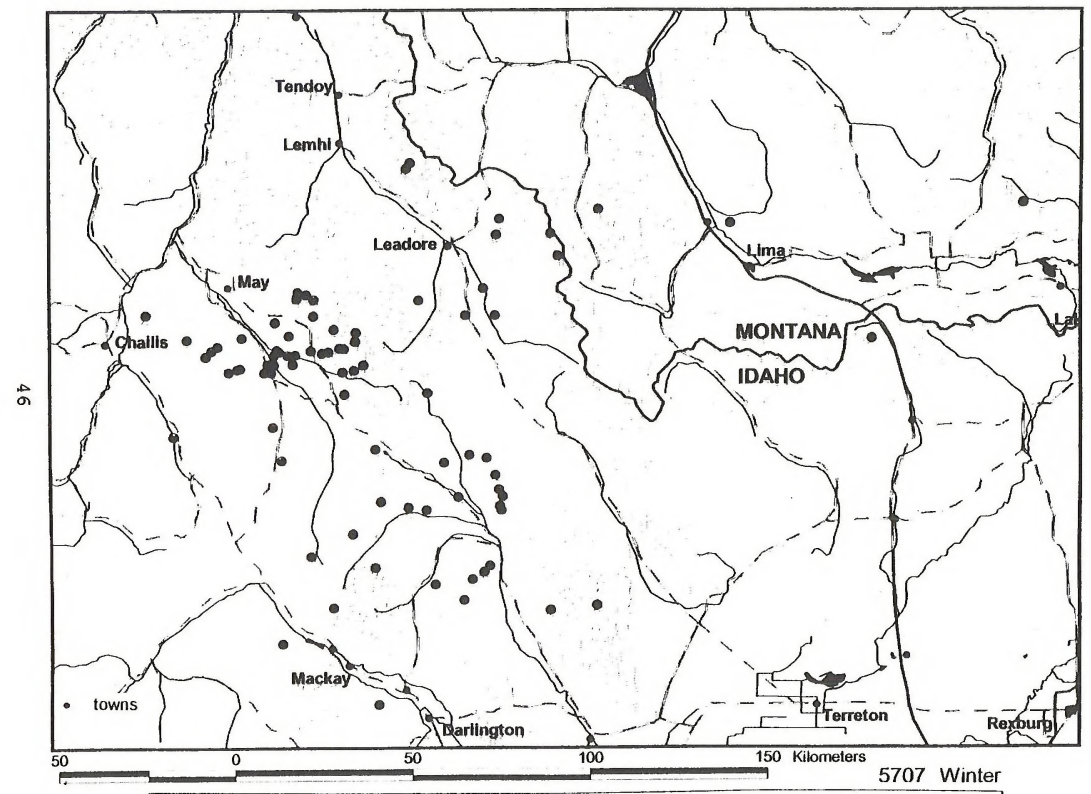

Figure 22. Range of a subadult female golden eagle (transmitter 5707 ) in the Intermountain West during winter 1995-96. 


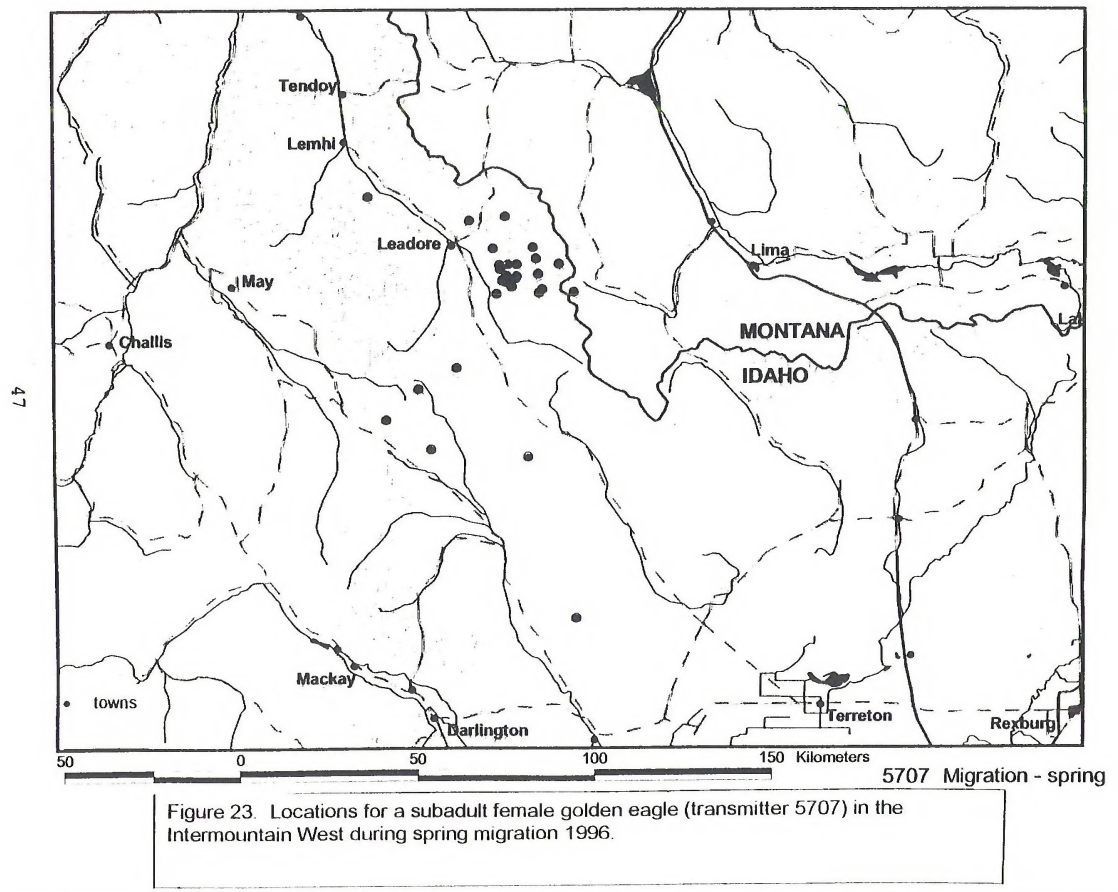




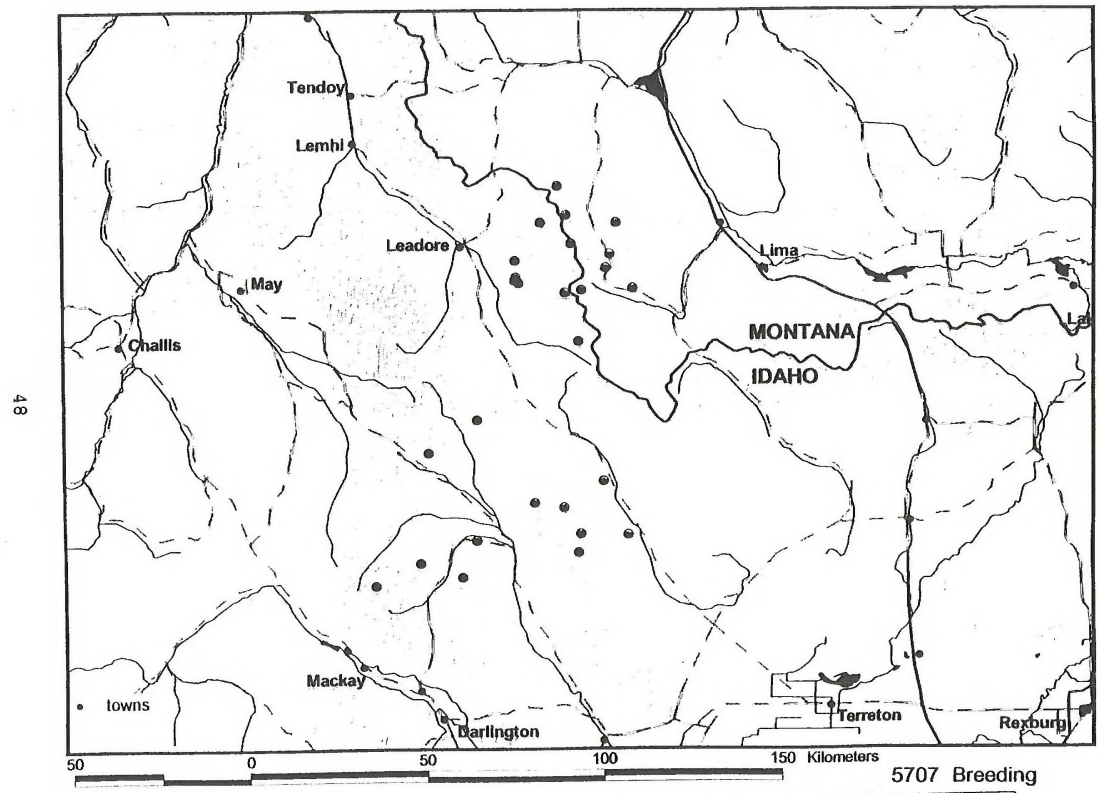

Figure 24. Home range of a subadult female golden eagle (transmitter 5707) in the intermountain West during breeding season 1996. 


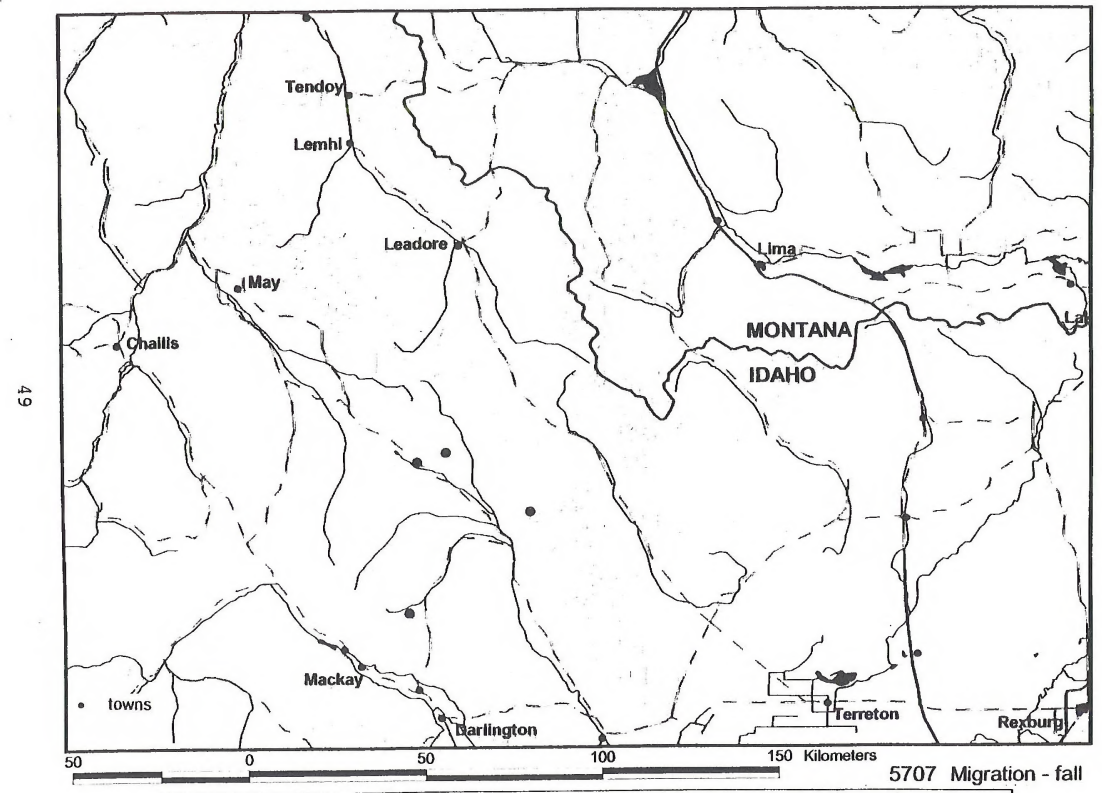

Figure 25. Locations for a subadult female golden eagle (Iransmitter 5707) in the Intermountain West during fall migration 1996. 


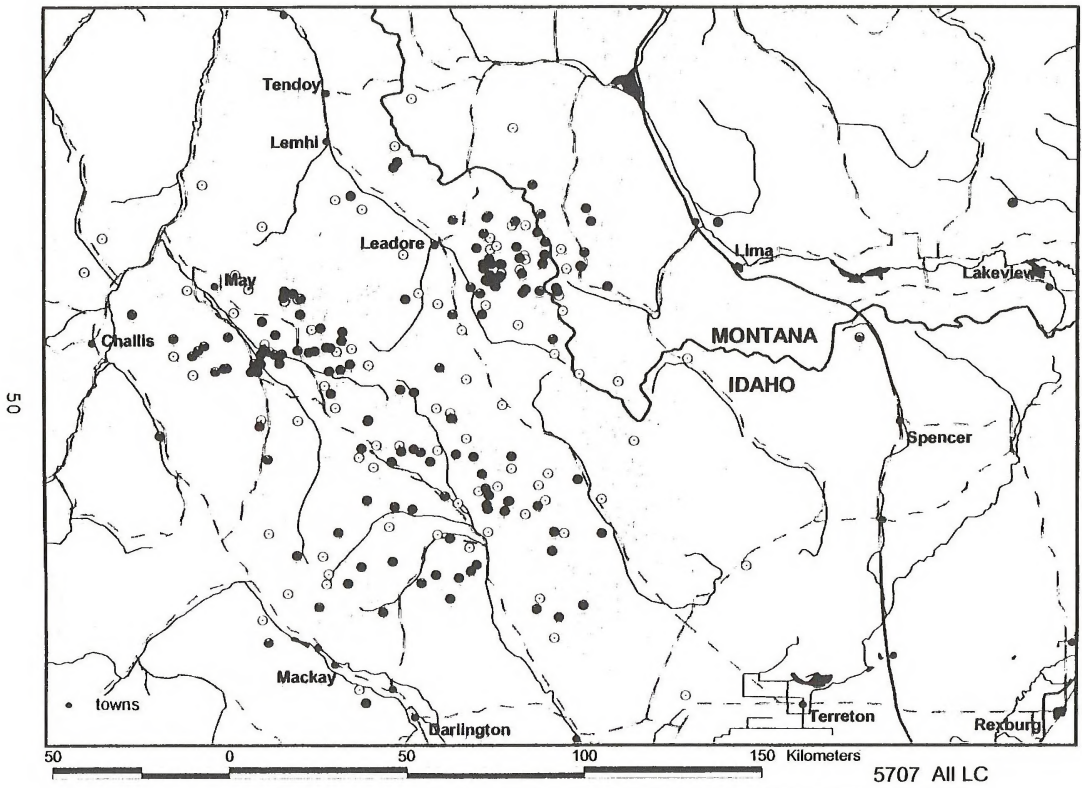

Figure 26. All locations for a subadult female golden eagle (transmitter 5707 ) in the Intermountain West from 8 January 1996 through 26 November 1996. 


\section{LITERATURE CITED}

Anonymous. 1992. FWS Regional News, Region 8 (Research). Endangered Species Technical Bulletin. 17(9-11):15.

Beyer, W.N., J.W. Spann, L. Sileo and J.C. Franson. 1988. Lead poisoning in six captive avian species. Arch. Environ. Contam. Toxicol. 17:121-130.

Bloom, P.H. 1987. Capturing and handling raptors, p. 290-294. In: B.A. GironPendleton, B.A. Millsap, K.W. Cline, and D.M. Bird [eds.], Raptor Management Techniques Manual. National Wildlife Federation, Washington, DC.

Brodeur, S., R. Dècarie, D.Bird, and M. Fuller. 1996. Complete migration cycle of golden eagles breeding in northern Quebec. Condor. 98:293-299.

Brown, B.T. 1992. Golden eagles feeding on fish. J. Raptor Res. 26(1):36-37.

Craig, E.H., T.H. Craig and L.R. Powers. 1986. Habitat use by wintering golden eagles and rough-legged hawks in southeastern Idaho. J. Raptor Res. 20(2):6971.

Craig, T.H., J.W. Connelly, E.H. Craig and T. Parker. 1990. Lead concentrations in golden and bald eagles. Wilson Bull. 102(1):130-133.

and E.H. Craig. 1995. Lead and mercury in golden and bald eagles in east central Idaho. 1990-1994. A progress report. BLM, State Office. Boise, ID.

and E.H. Craig. 1996. Lead and mercury in golden and bald eagles in east central Idaho. 1990-1995. A progress report. BLM, Salmon District Office. Salmon, ID.

Dietz, D.D., D.E. McMilland, and P. Mushak. 1979. Effects of chronic lead administration on acquisition and performance of serial position sequences by pigeons. Toxicol. Appl. Pharmacol. 47:377-384.

Dunstan, T.C., J.H. Harper, and K.B. Phipps. 1978. Habitat use and hunting strategies of prairie falcons, red-tailed hawks, and golden eagles. U.S. Dept. of the Interior, BLM, Denver, CO.

Eakle, W.L. and T.G. Grubb. 1986. Prey remains from golden eagle nests in central Arizona. Western Birds 17:87-89.

Eisler, R. 1988. Lead hazards to fish, wildlife and invertebrates: A synoptic review. US Fish Wildl. Serv. Biol. Rep. 85(1.14), 134 pp. 
1987. Mercury hazards to fish, wildlife and invertebrates: A synoptic review. US Fish \& Wildl. Serv. Biol. Rep. 85(1.10), 90 pp.

Feierabend, J.S. and O. Myers. 1984. A national summary of lead poisoning in bald eagles and waterfow. $90 \mathrm{pp}$. Avail. from Nat. Wildl. Fed., 1412 Sixteenth St. NW, Washington, D.C. 20036.

Franson, J.C., L. Sileo and N.J. Thomas. 1995. Causes of eagle deaths, p. 68. In: E.T. LaRoe, G.S. Farris, C.E. Puckett, P.D. Doran, M.J. Mac [eds.], Our living resources. A report to the nation on the distribution, abundance, and health of U.S. plants, animals, and ecosystems. USDI-NBS. US Government Printing Office. Stock \#024-010-00708-7. Washington, DC. 20402.

Harmata, H.R. and M. Restani. 1995. Contaminants in blood of vernal migrant bald and golden eagles. Intermountain Journal Sci. 1(1):1-15.

Henny, C.J., L.J. Blus, D.J. Hoffman and R.A. Grove. 1994. Lead in hawks, falcons and owis downstream from a mining site on the Coeur d'Alene River, Idaho. Environ. Monitor. and Assess. 29:267-288.

L.J. Blus, D.J. Hoffman, R.A. Grove and J.S. Hatfield. 1991. Lead accumulation and Osprey production near a mining site on the Coeur d'Alene River, Idaho. Arch. Environ. Contam. Toxicol. 21:415-424.

Hodder, K.H., R.E. Kenward, S.S. Walls and R.T. Clarke. 1998. Estimating core ranges: a comparison of techniques using the common buzzard (Buteo buteo). J. Raptor Res. 32(2):82-89.

Hoffman, D.J., O.H. Pattee, S.N. Wiemeyer and B. Muihern. 1981. Effects of lead shot ingestion on $\delta$-aminolevulinic acid dehydratase activity, hemoglobin concentration, and serum chemistry in bald eagles. J. Wildl. Diseases 17(3):423-431.

Hoffman, S.W., J.C. Bednarz, and W.R. DeRagon. 1996. Trends in counts of migrant hawks in western North America, 1977-1995. Paper presented at: $114^{\text {th }}$ meeting of the AOU and 1996 annual meeting of the Raptor Research Foundation. 1317 August. Boise, Idaho.

Kaiser, T.E., W.L. Reichel, L.N. Locke, E. Cromartie, A.J. Krynitsky, T.G. Lamont, B.M.Mulhern. R.M. Prouty, C.M. Stafford and D.M. Swineford. 1980. Organochlorine pesticide, PCB, and PBB residues and necropsy data for Bald Eagles from 29 states--1975-77. Pesticides Monitor. J. 13(4):145-149. 
Kendall, R.J. 1982. Wildlife toxicology. Integrated field and laboratory studies using selected model species might lead to ways of quantifying adverse effects of chemical contaminants. Environ. Sci. Technol. 16(8):448A-453A.

, P.F. Scanlon, and R.T. Di Giulio. 1982. Toxicology of ingested lead shot in ringed turtle doves. Arch. Environ. Contam. Toxicol. 11:259-263.

Kenward, R.E., AND K.H. Hodder. 1995. Ranges V. software for analyzing animal location data. Inst. Terrestrial Ecology, Wareham, UK.

Kramer, J.L. and P.T. Redig. 1997. Sixteen years of lead poisoning in eagles, 198095: an epizootiologic view. J. Raptor Res. 31:327-332.

Marzluff, J.M., S.T. Knick, M.S. Vekasy, L.S. Schueck, and T.J. Zarriello. 1997. Spatial use and habitat selection of golden eagles in southwestern Idaho. The Auk. 114:673-687.

Nelson, T.A., C. Mitchell and C. Abbot. 1989. Lead-shot ingestion by bald eagles in western Arkansas. Southwest. Nat. 34(2):245-249.

Oberg, P.M. 1970. Between these mountains. Exposition Press Inc. Jericho, New York $199 \mathrm{pp}$.

Odum, E.P. 1971. Fundamentals of ecology. Saunders, Philadelphia, Pennsylvania. $574 \mathrm{pp}$.

Pac, D.F., K.L. Hamlin, and R.M. Desimone. 1995. Mortality of bull elk and buck mule deer in southwestern Montana: comparative implications for management. Proceedings of the Western States and Provinces 1995 Joint Deer and Elk Workshop. Sun Valley, Idaho. Idaho Dept. of Fish and Game. Abstract No. 42.

Pattee, O.H., S.N. Wiemeyer, B.M. Mulhern, L. Sileo and M.W. Carpenter. 1981. Experimental lead-shot poisoning in Bald Eagles. J. Wildl. Manage. 45(3):806810.

and S.K. Hennes. 1983. Bald Eagles and Waterfow: The Lead Shot Connection. Transactions of the $48^{\text {th }} \mathrm{N}$. American Wildlife \& Natural Resources Conf. Wildlife Management Inst., Washington, D.C. pp. 230-237.

P.H. Bloom, J.M. Scott and M.R. Smith. 1990. Lead hazards within the range of the California condor. Condor. 92:931-937.

Platt, J.B. 1976. Bald eagles wintering in a Utah desert. Amer. Birds 30(4):783-788. 
Redig, P.T. 1993. Medical management of birds of prey. A collection of notes on selected topics. Third Edition, revised. The Raptor Center, Univ. of MN, St. Paul, MN 55108. Chapter 18:151-156.

Reichel, S.K. Schmeling, E. Cromartie, T.E.Kaiser, A.J. Krynitsky, T.G. Lamont, B.M. Mulhern, R.M. Prouty, C.J. Stafford and D.M. Swineford. 1984. Pesticide, PCB, and lead residues and necropsy data for Bald Eagles from 32 states - 1978-81. Environ. Monit. and Assess. 4(4):395-403.

Schueck, L.S., J.M. Marzluff, M. Vekasy, M.R. Fuller and W.S. Seegar. 1994. Migration routes and winter home ranges of golden eagles determined by satellite and conventional radiotelemetry. Paper presented at the $112^{\text {th }}$ stated meeting of the American Ornithologists' Union. June 1994.

Task Group on Metal Accumulation. 1973. Accumulation of Toxic Metals with Special Reference to Their Absorption, Excretion and Biological Half-Times. Environ. Physiol. Biochem. 3:65-107.

Terres, J.K. 1980. Encyclopedia of North American Birds. Alfred A Knopf. New York. $1109 \mathrm{pp}$.

Unsworth, J.W., L. Kuck, M.D. Scott, and E.O. Garton. 1993. Elk mortality in the Clearwater drainage of northcentral Idaho. J. Wildl. Manage. 57:495-502.

USF\&WS. 1985. Draft Supplemental Environmental Impact Statement on the Use of Lead Shot for Hunting Migratory Birds in the United States. DES 85-83. USF\&WS. Washington, D.C.

Wiemeyer, S.N., R.W. Frenzel, R.G. Anthony, B.R. McClelland and R.L. Knight. 1989. Environmental contaminants in blood of western Bald Eagles. J. Raptor Res 23(4):140-146. 


\section{APPENDIX A}

Duty cycles of 6 golden eagles tracked via satellite in east central Idaho from January 1996 through November 1997. Transmitter numbers identify the duty cycles of individual eagles. 
Duty Cycle 5709

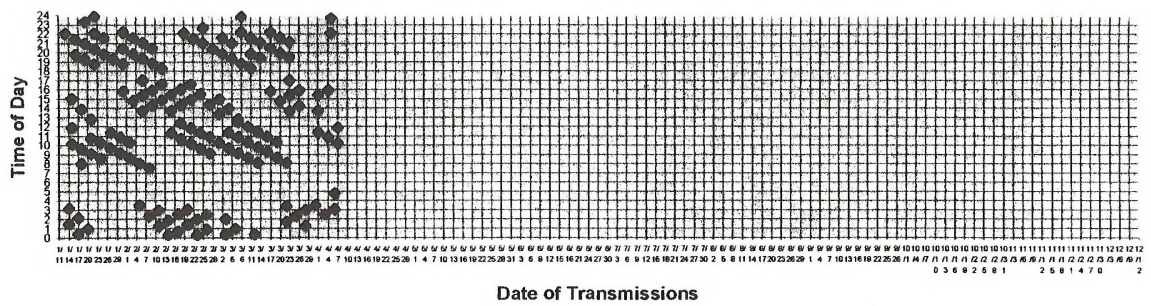


Duty Cycle 5727

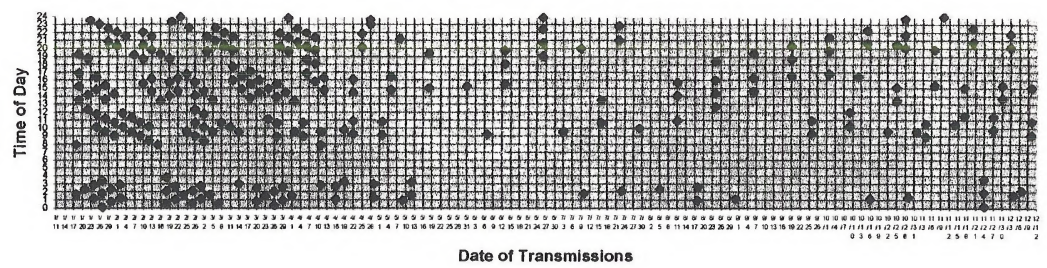

Duty Cycle 5727 cont.

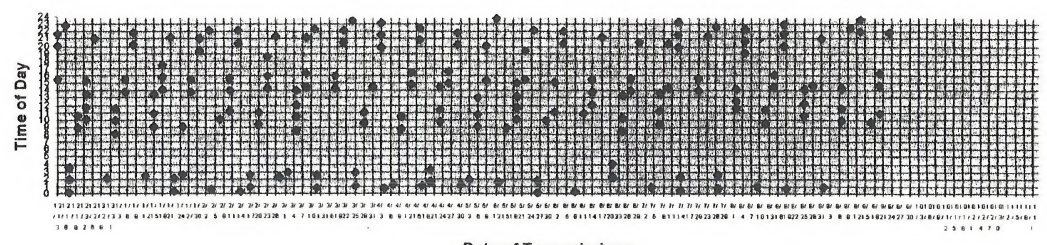

Date of Tranmissions 
Duty Cycle 5726

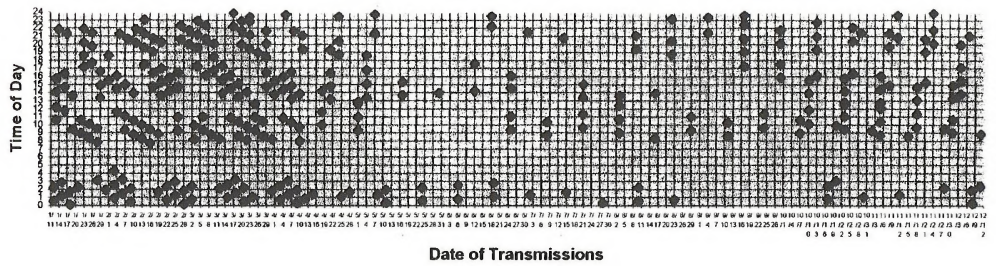

Duty Cycle 5726 cont.

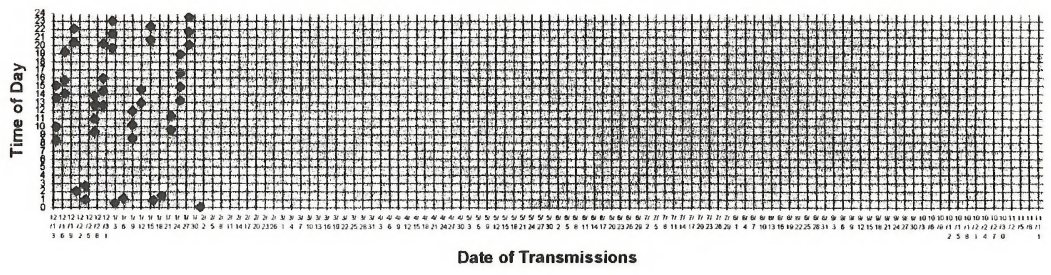


Duty Cycle 5699

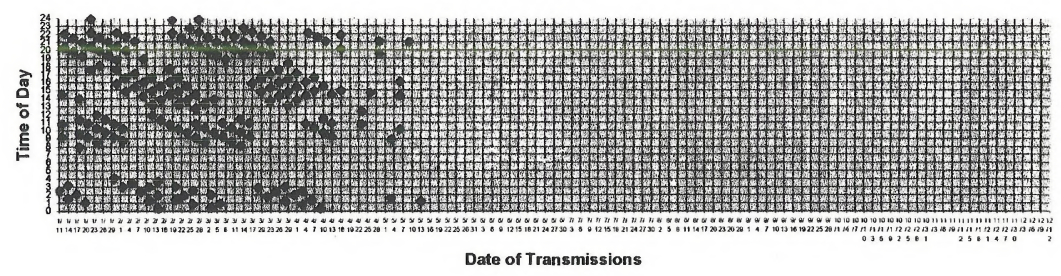

Duty Cycle $\mathbf{5 7 0 7}$

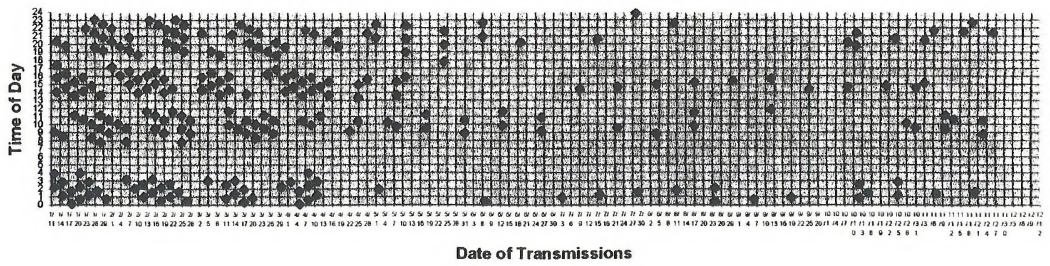


Duty Cycle $\mathbf{5 7 3 8}$

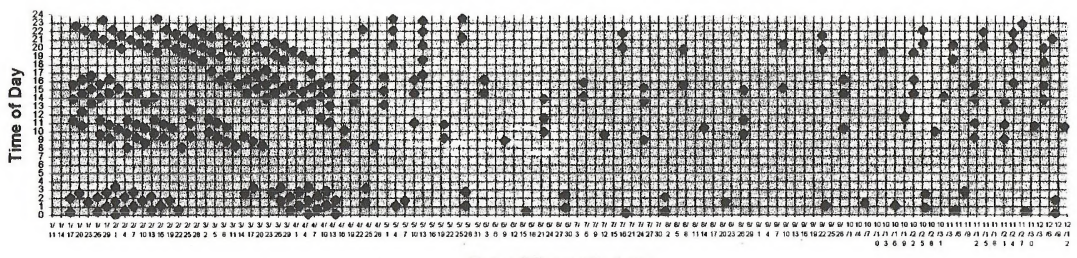

Date of Transmissions

Duty Cycle 5738 cont.

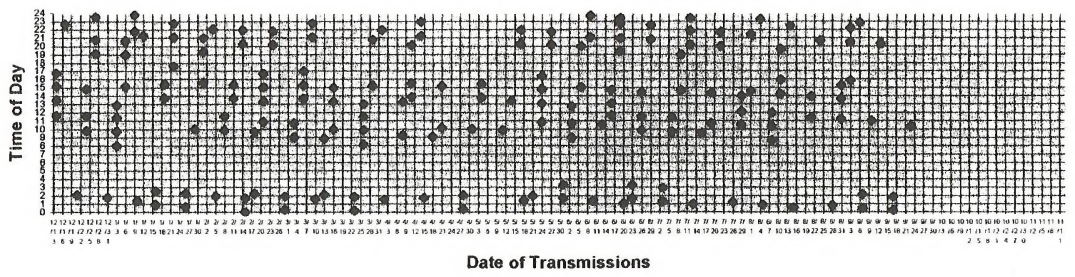




\section{APPENDIX B}

Individual records of distance moved between location estimates for 6 golden eagles tracked via satellite telemetry during 1995-1996. Distance moved since the previous location estimate is an average of all distances in an individual day, for days on which more than one location were received. 


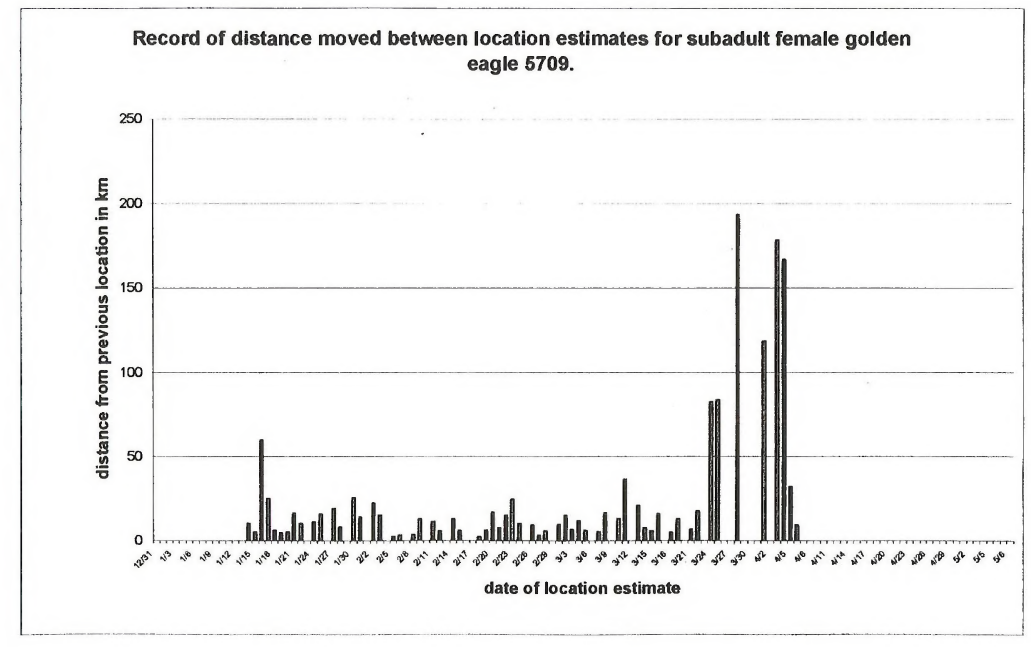




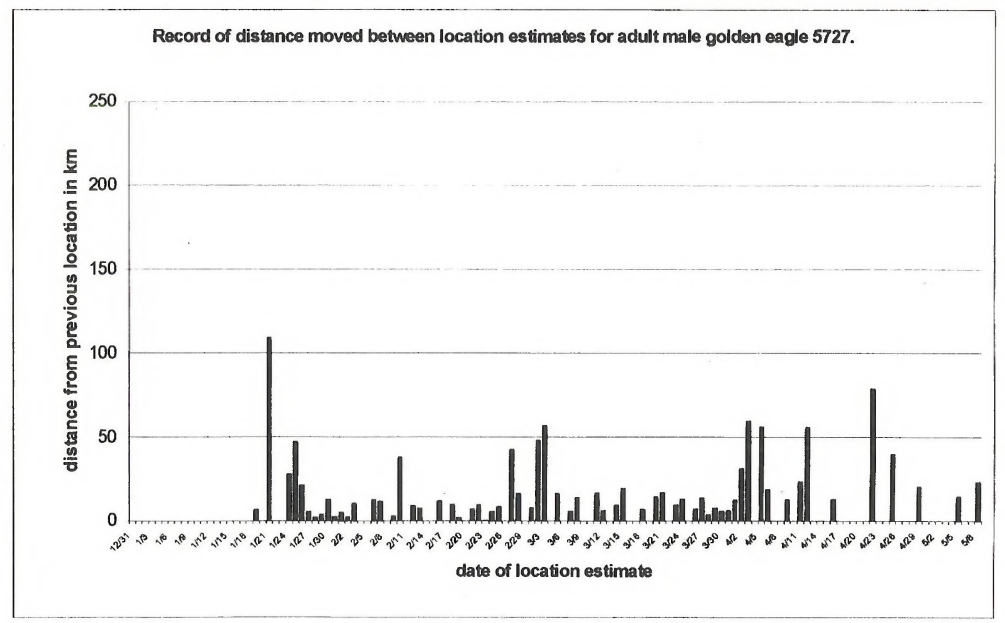




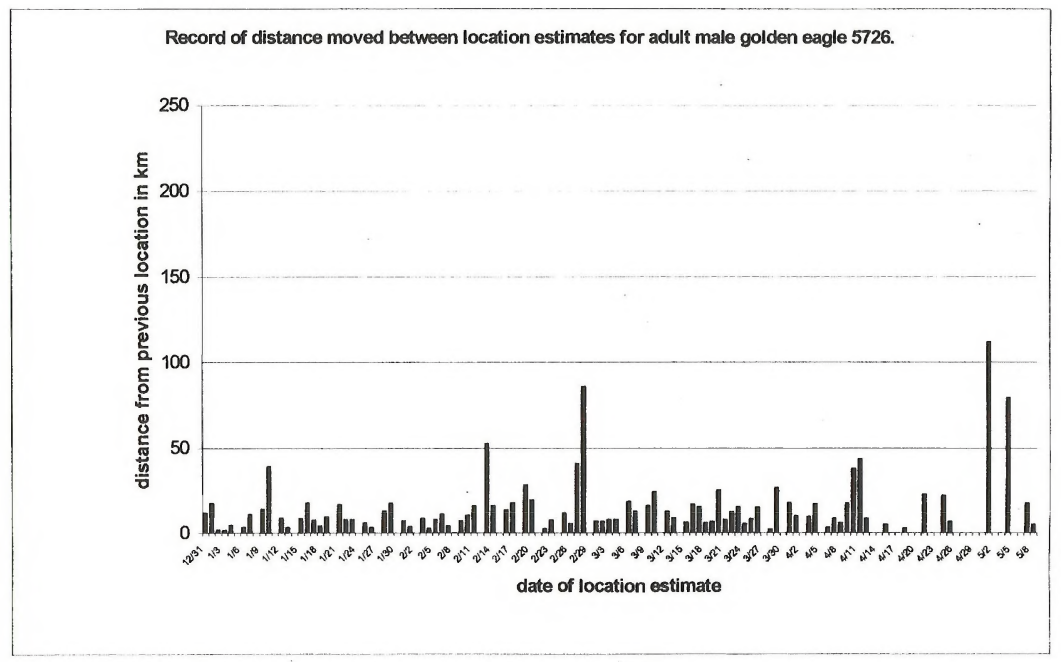




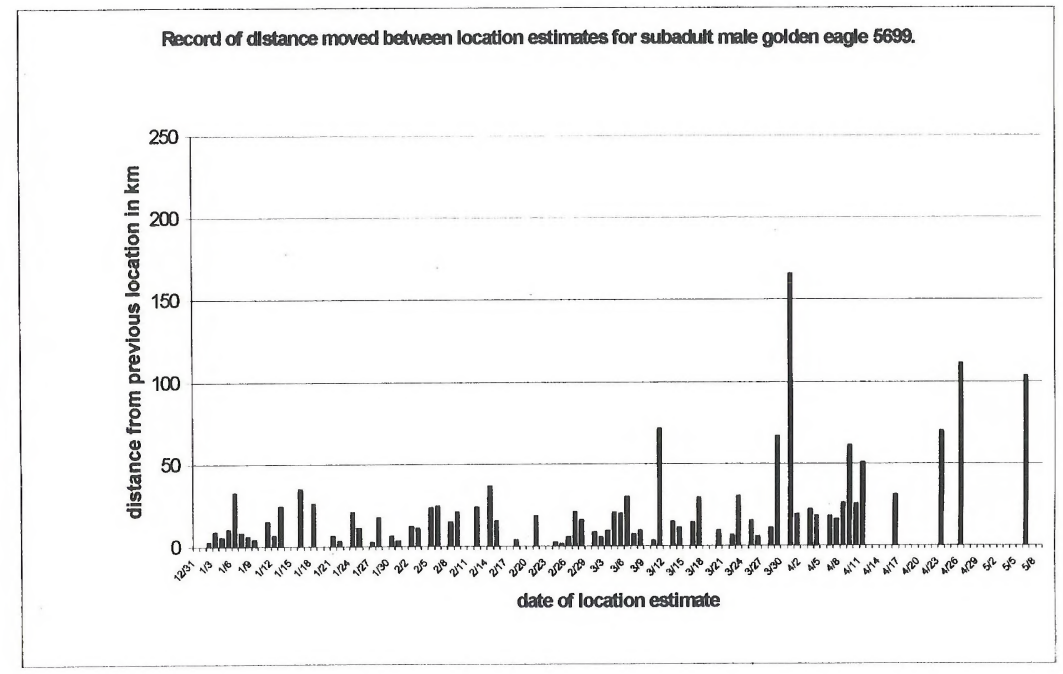




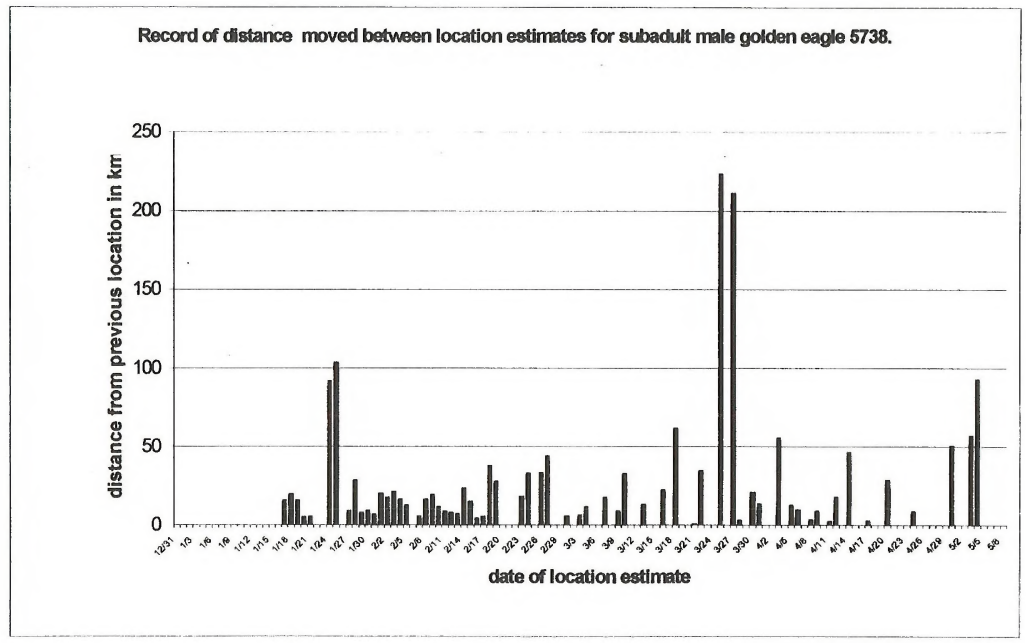




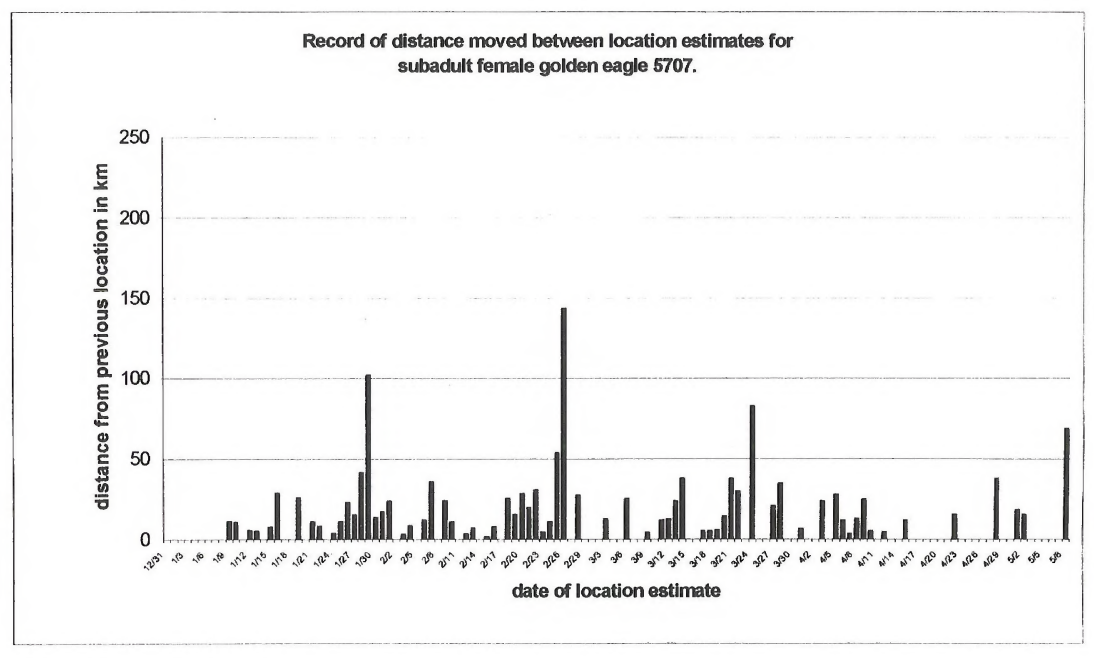




\section{APPENDIX C}

Monthly range maps of 6 golden eagles tracked via satellite from winter 1995-96 through winter 1996-97. 
Monthly range maps for adult, female golden eagle 5709; January through March 1996. 


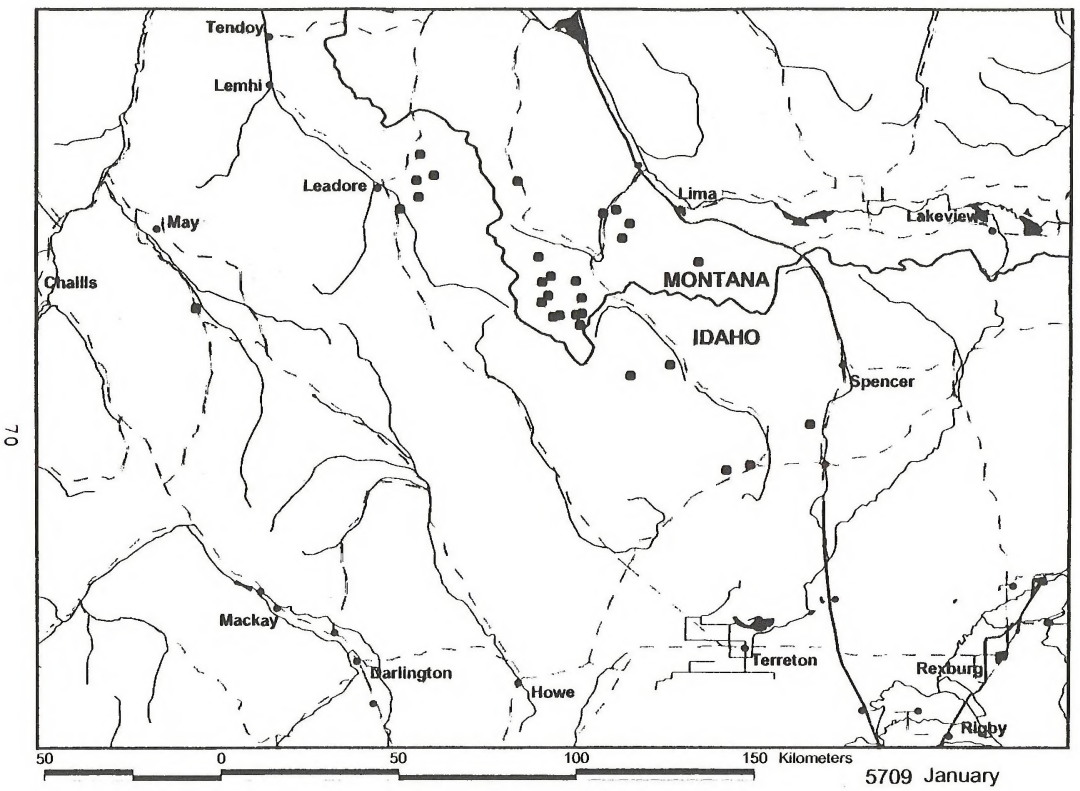

00000000000000000000000000000000000000000000000000000 


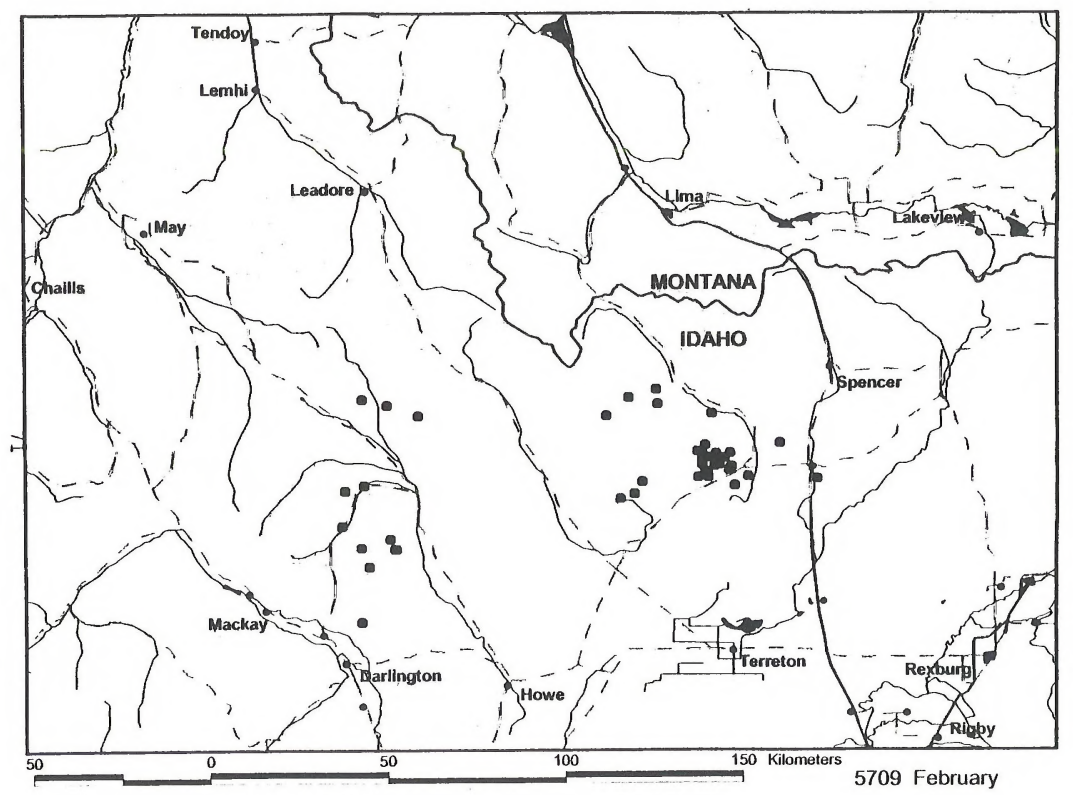




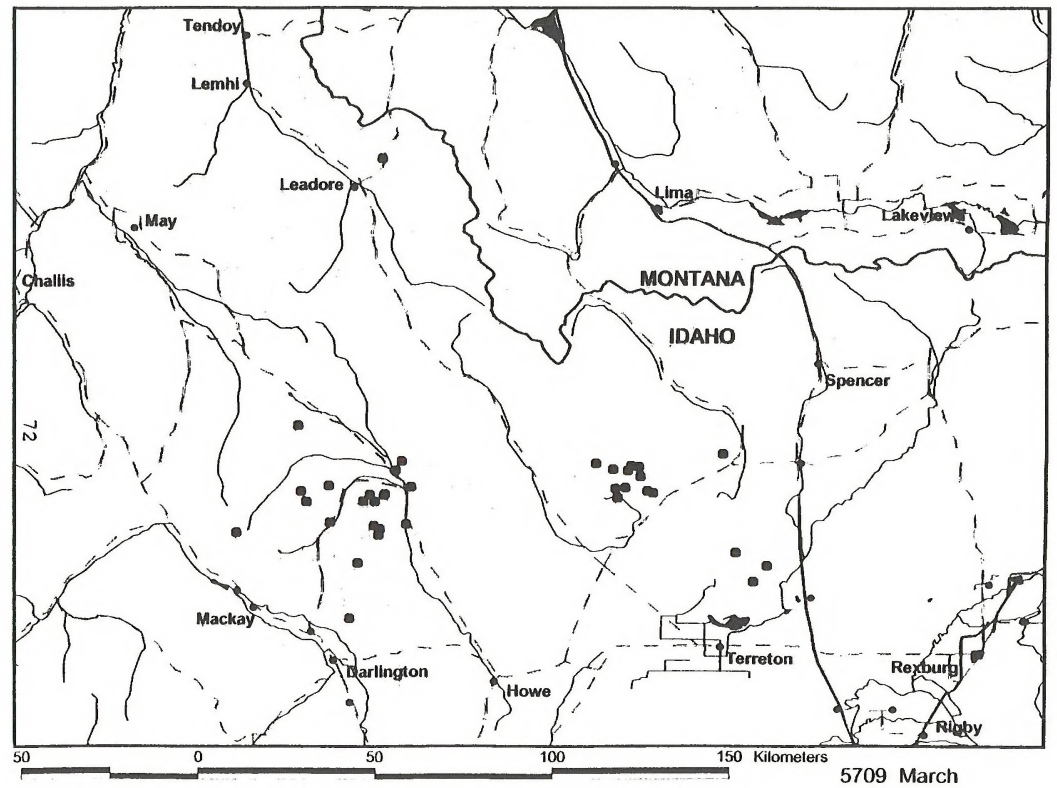

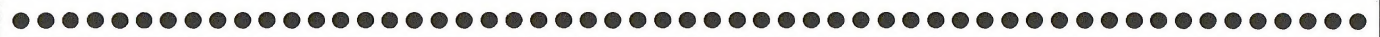


Monthly range maps for aduit, male golden eagle 5727; January through December 1996. 


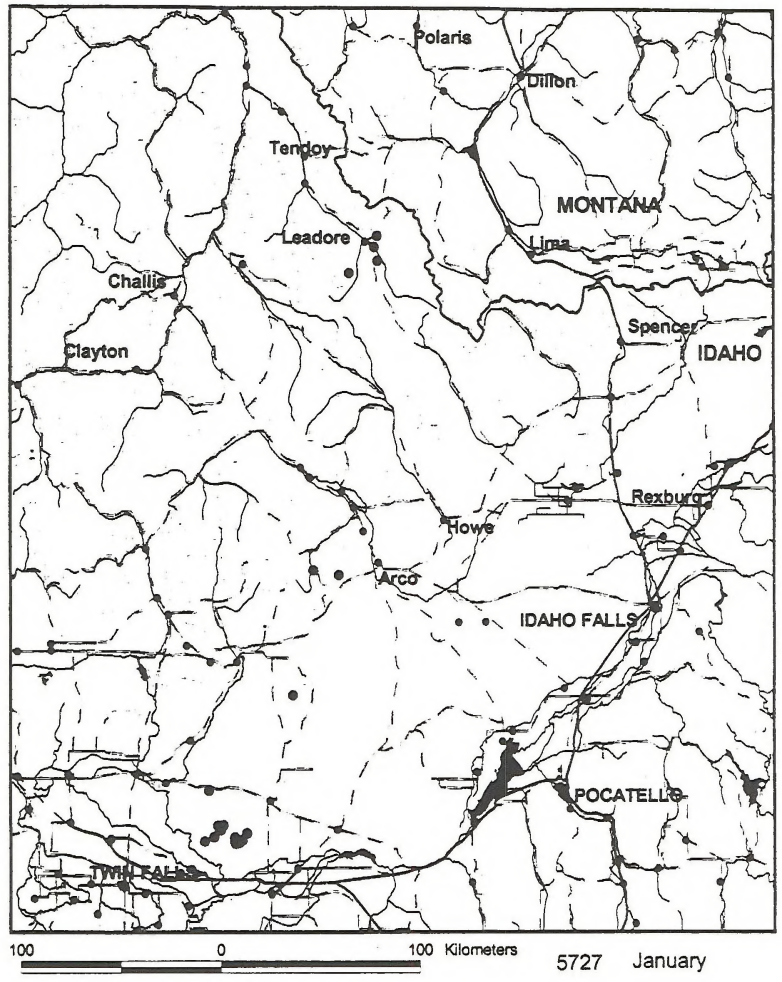




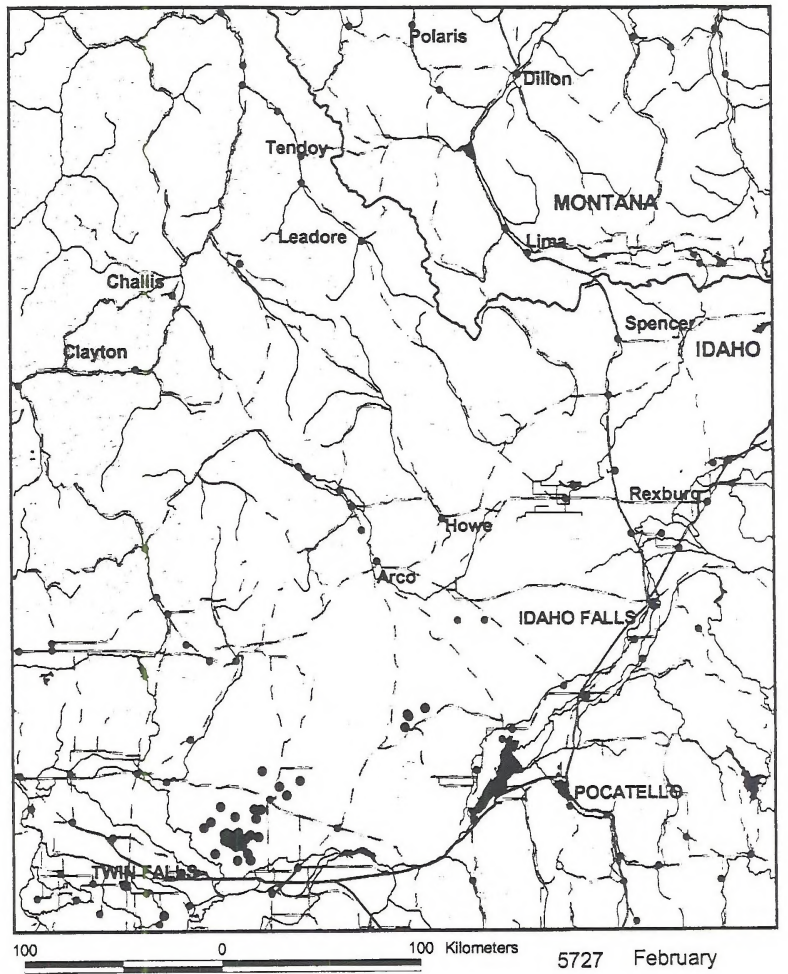




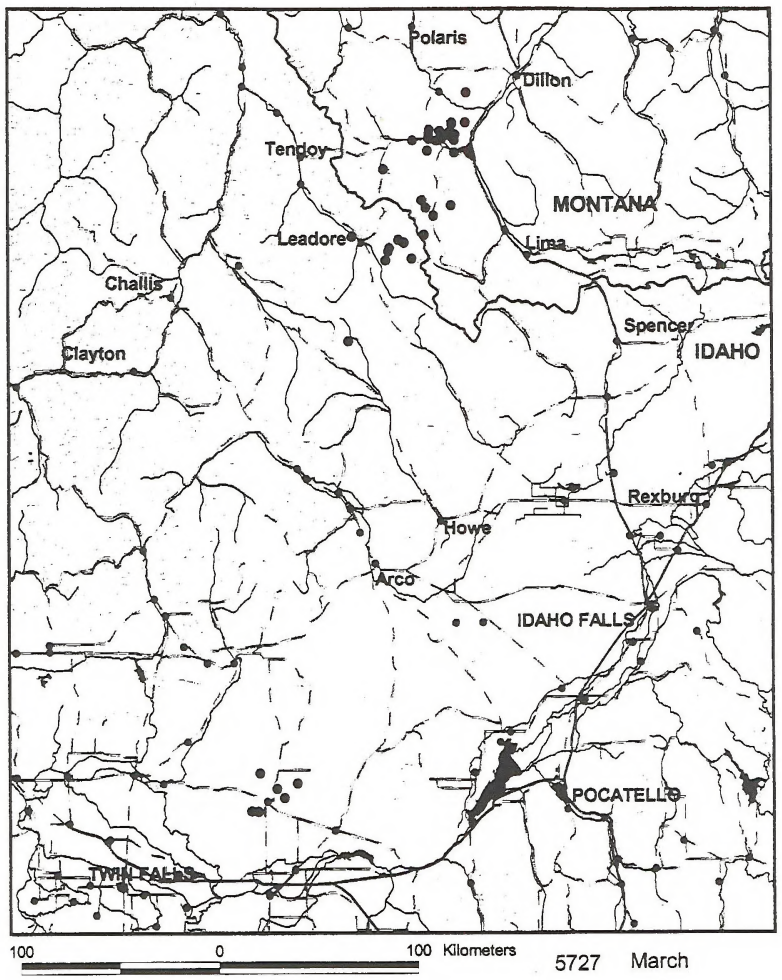




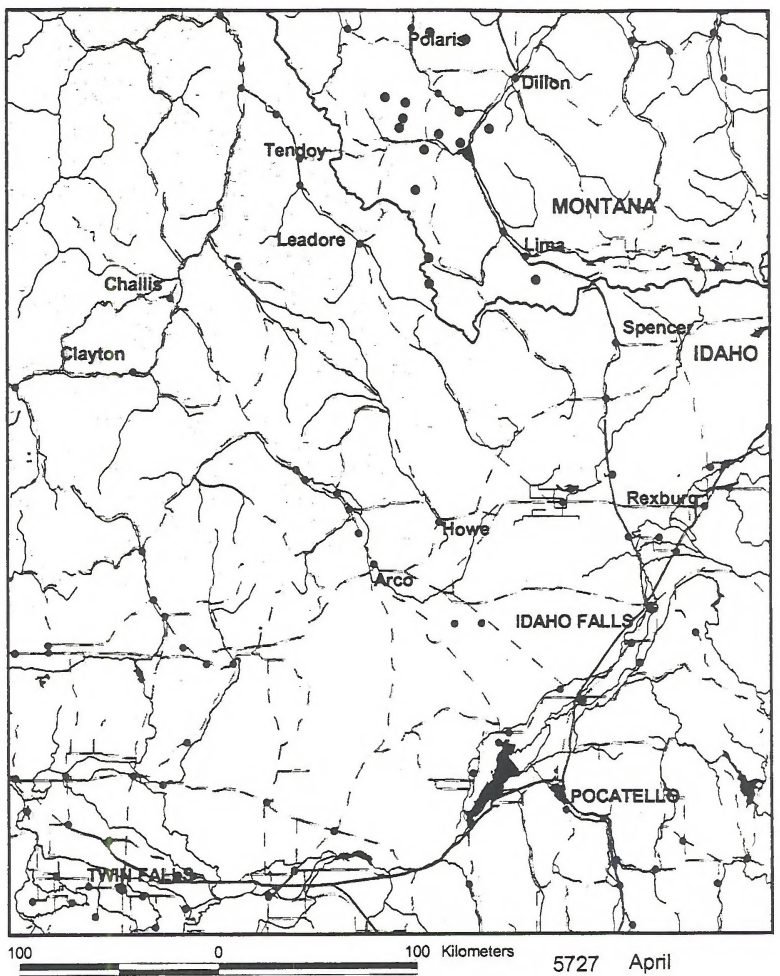




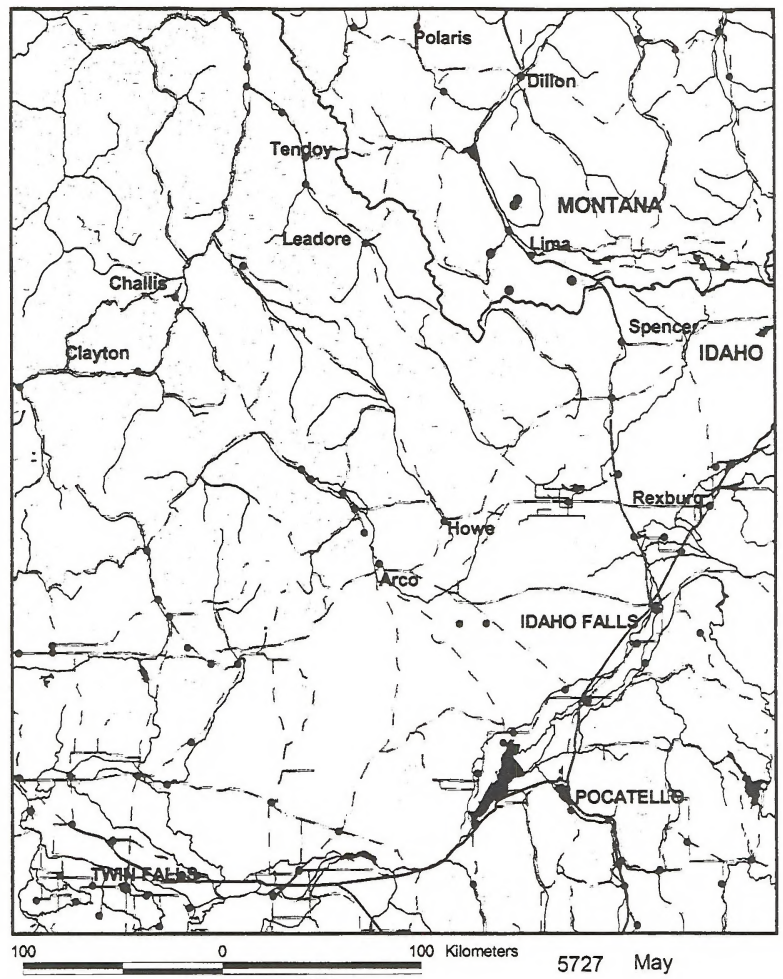




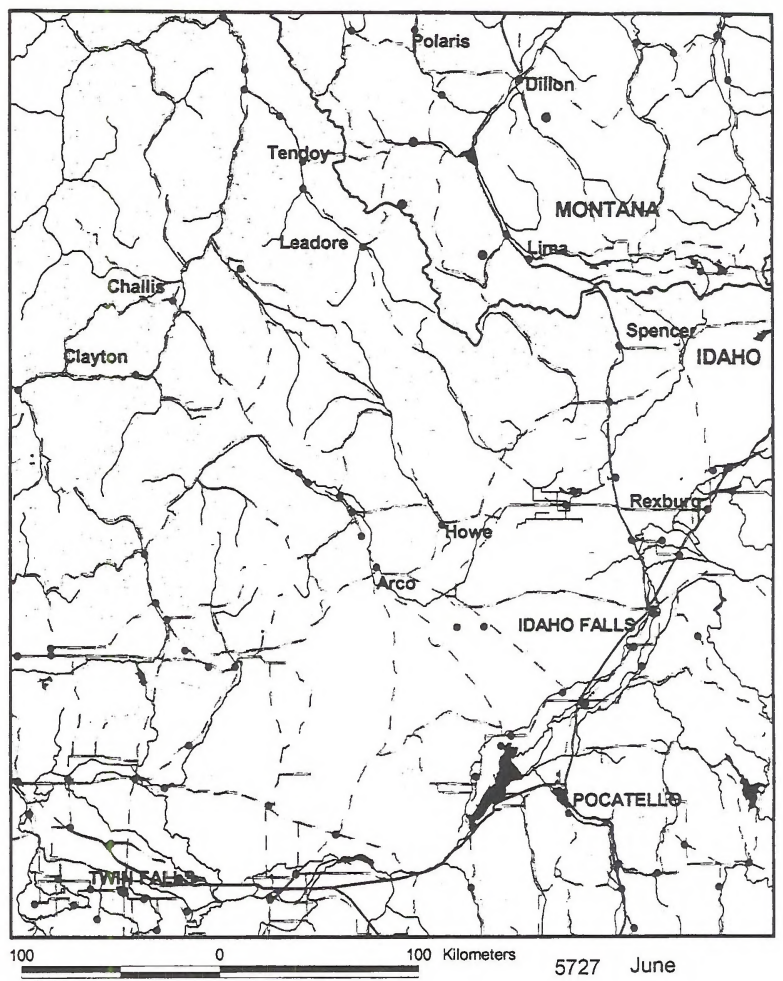




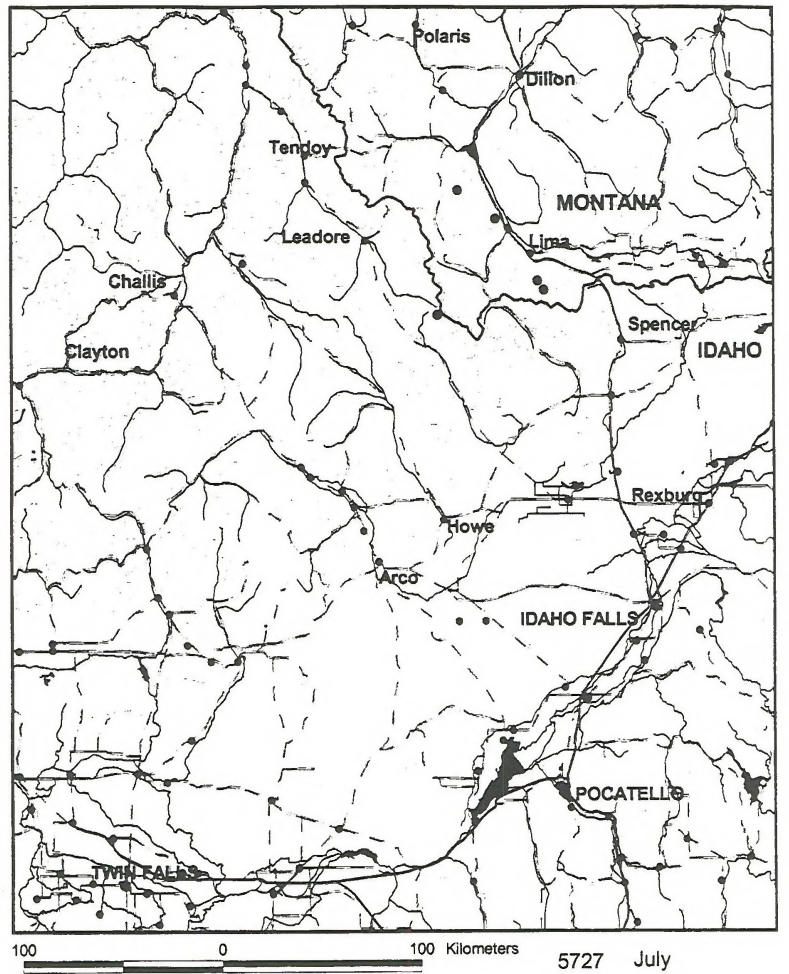




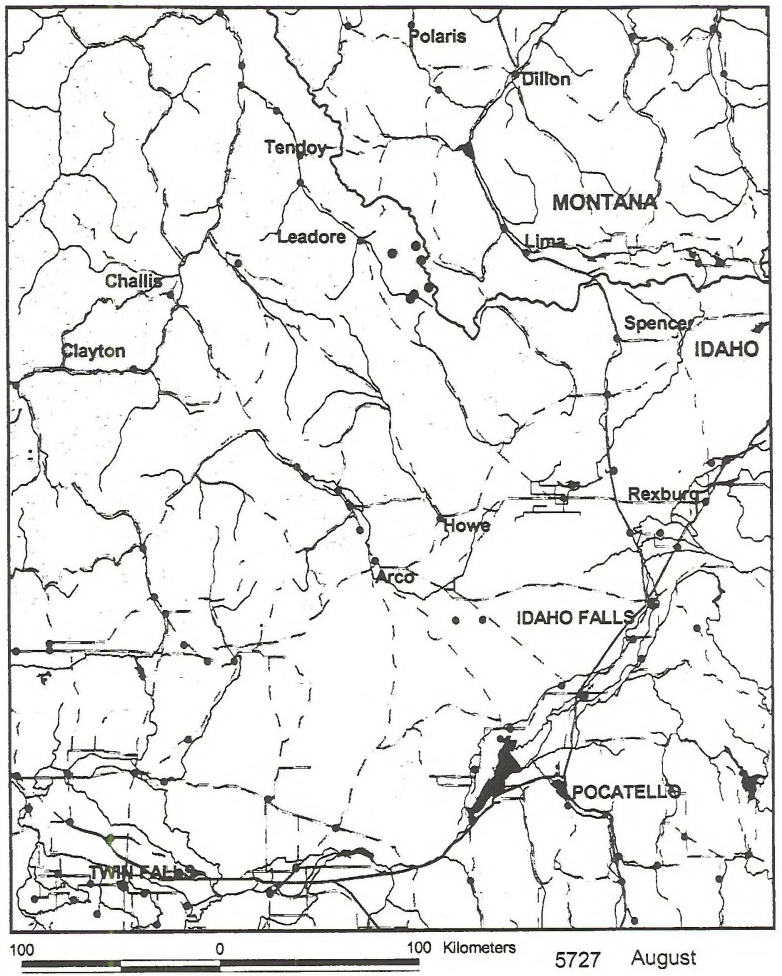




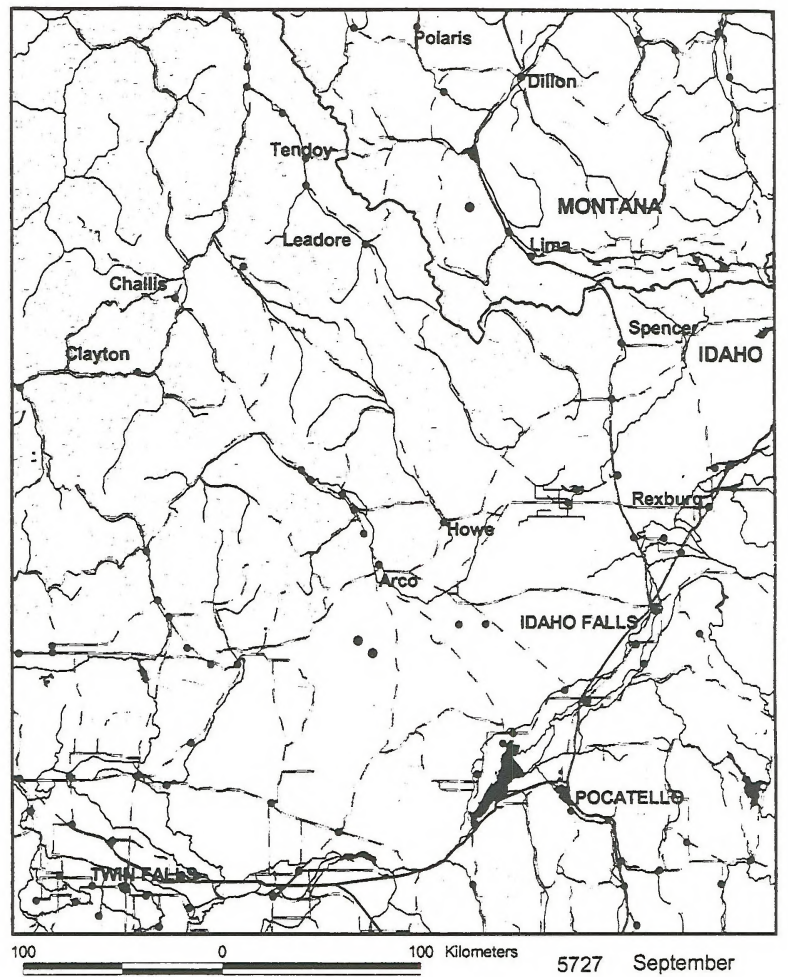




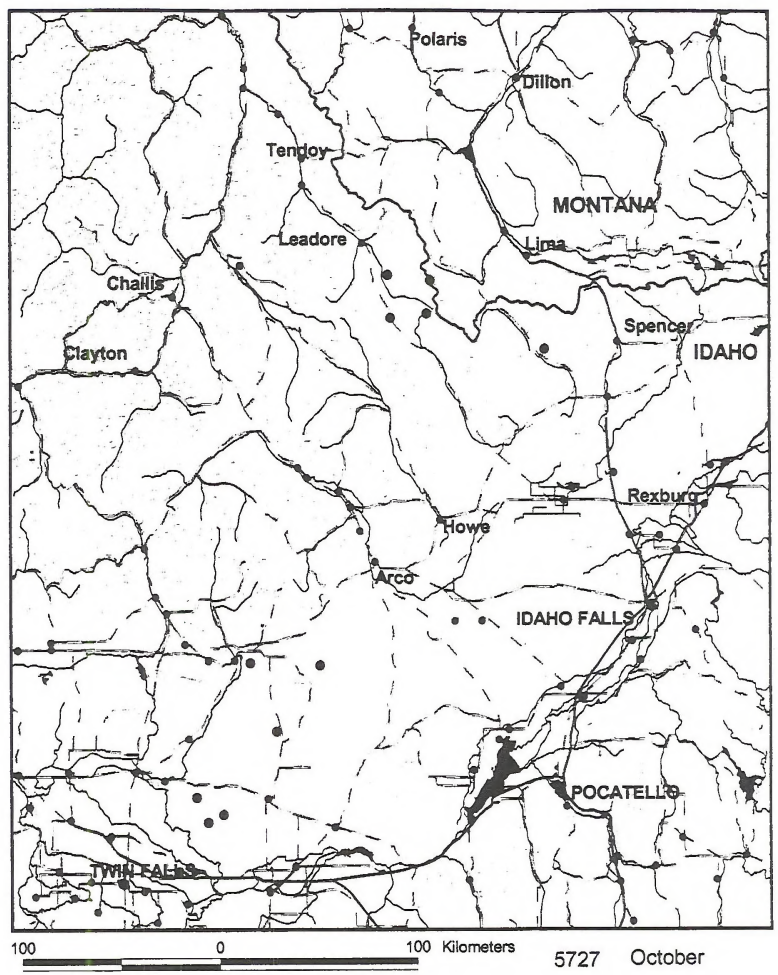




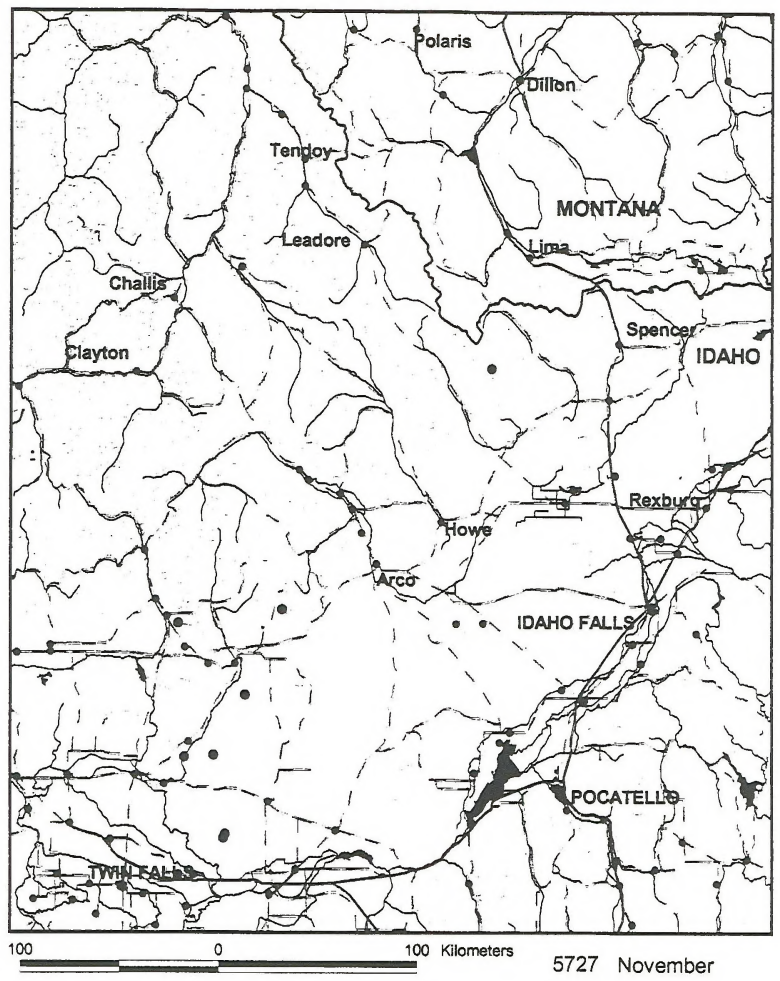




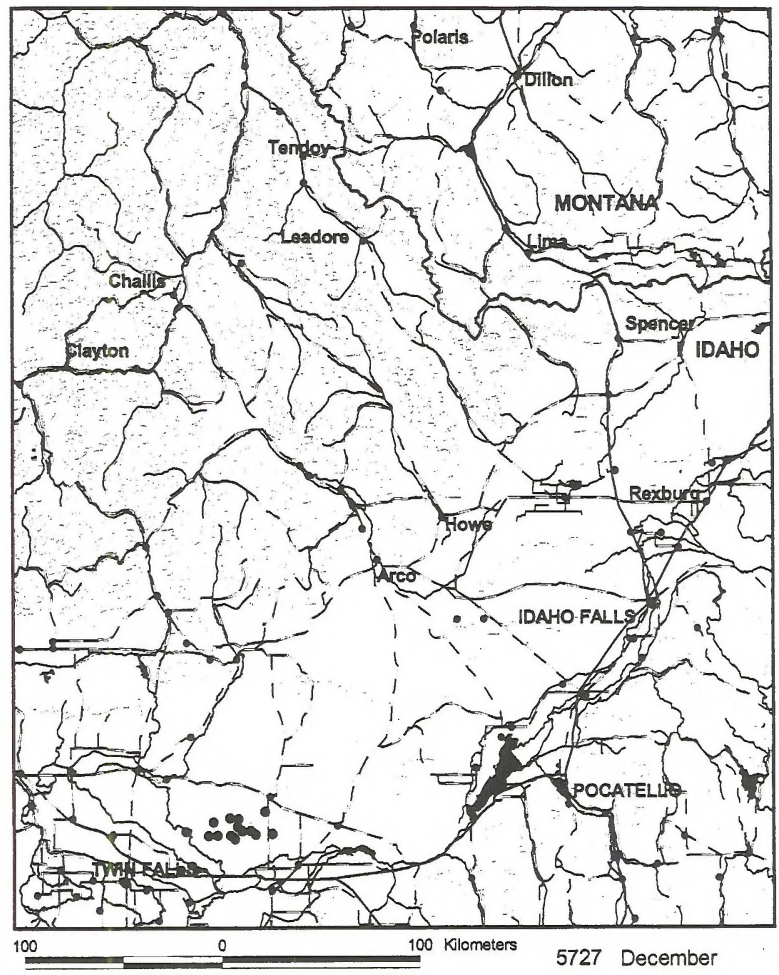


Monthly range maps for adult, male golden eagle 5726; December 1995 through December 1996. 


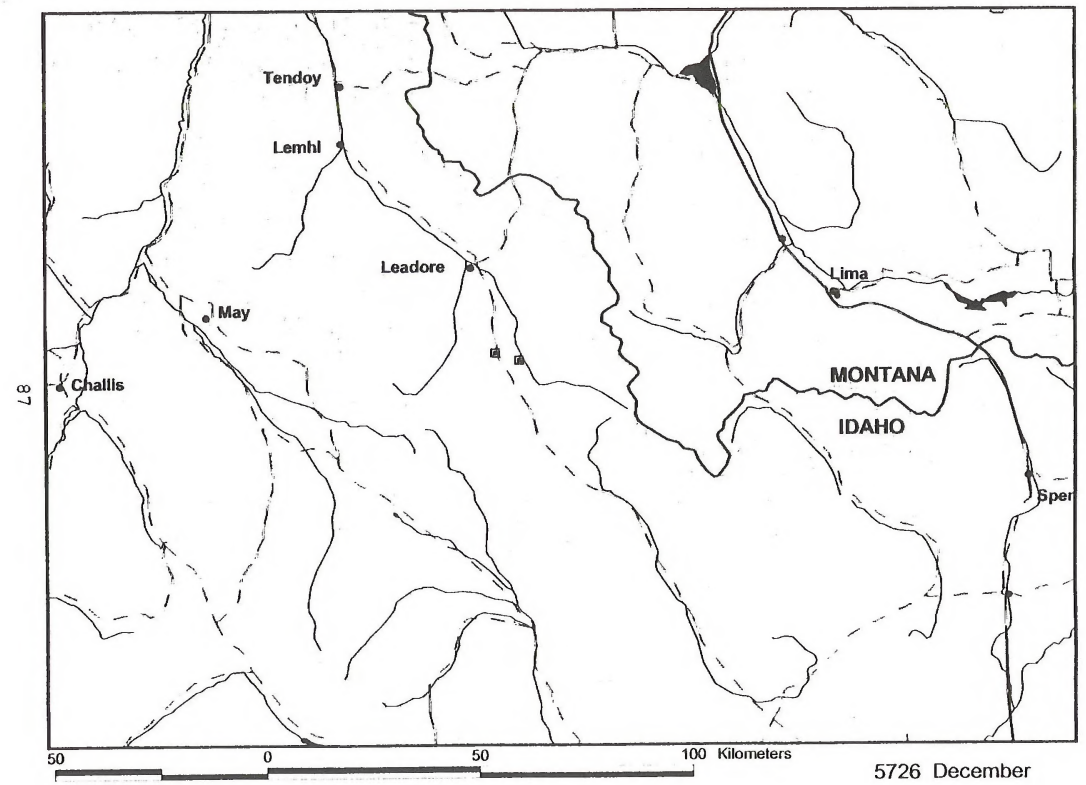




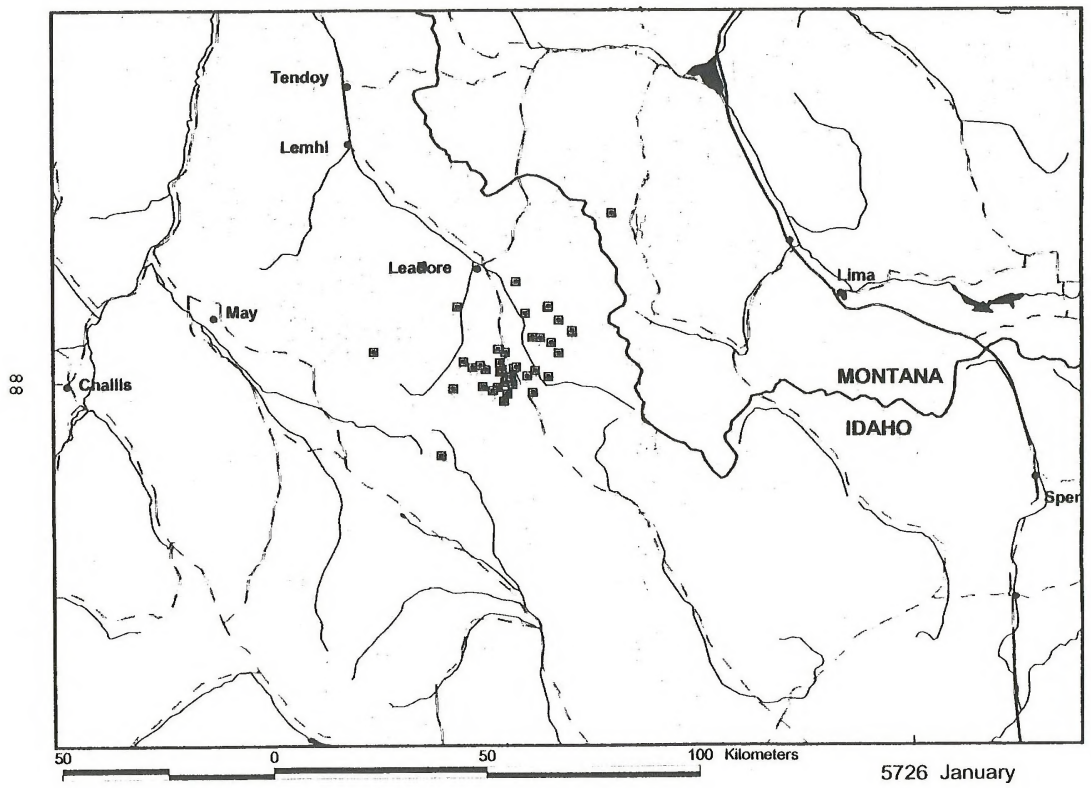

000000000000000000000000000000000000000000000000000000 


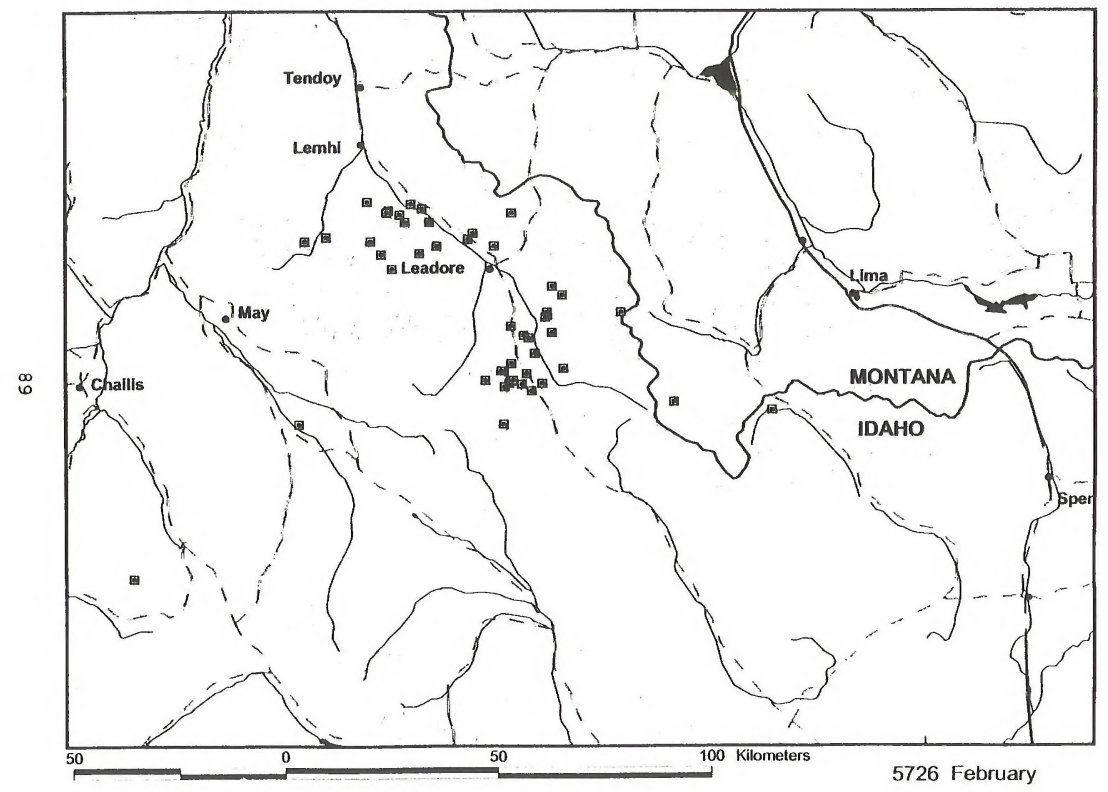




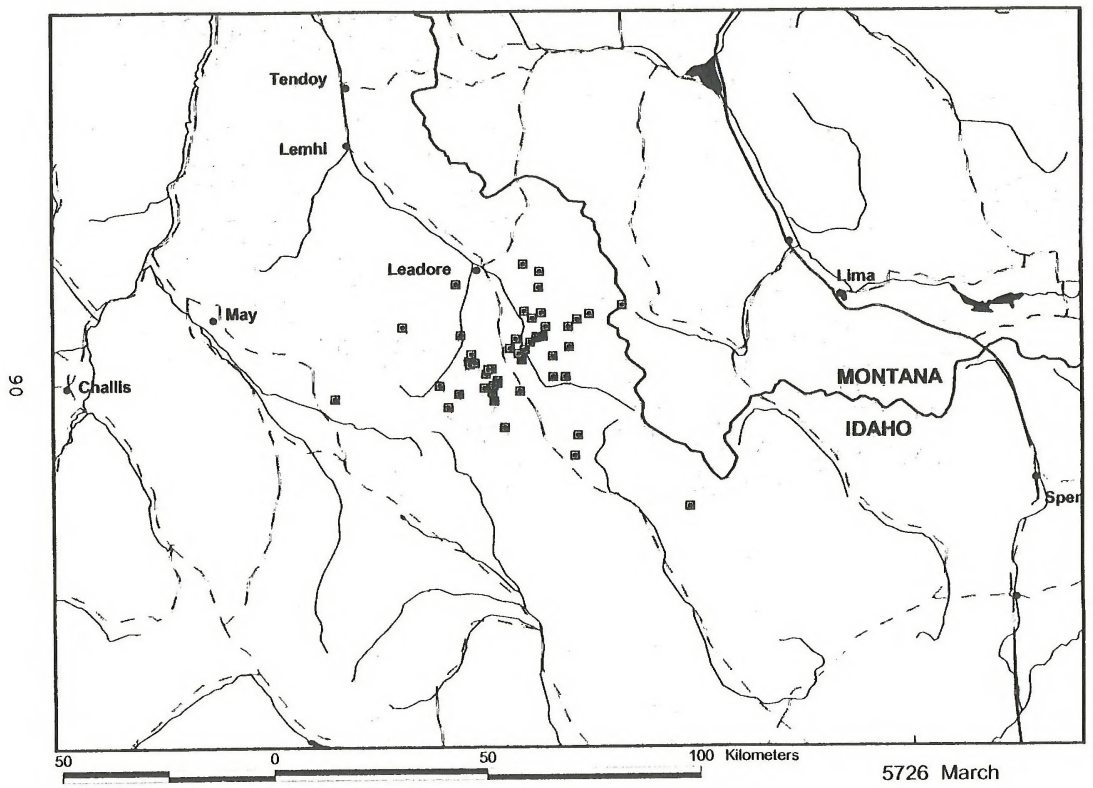

0000000000000000000000000000000000000000000000000000000 


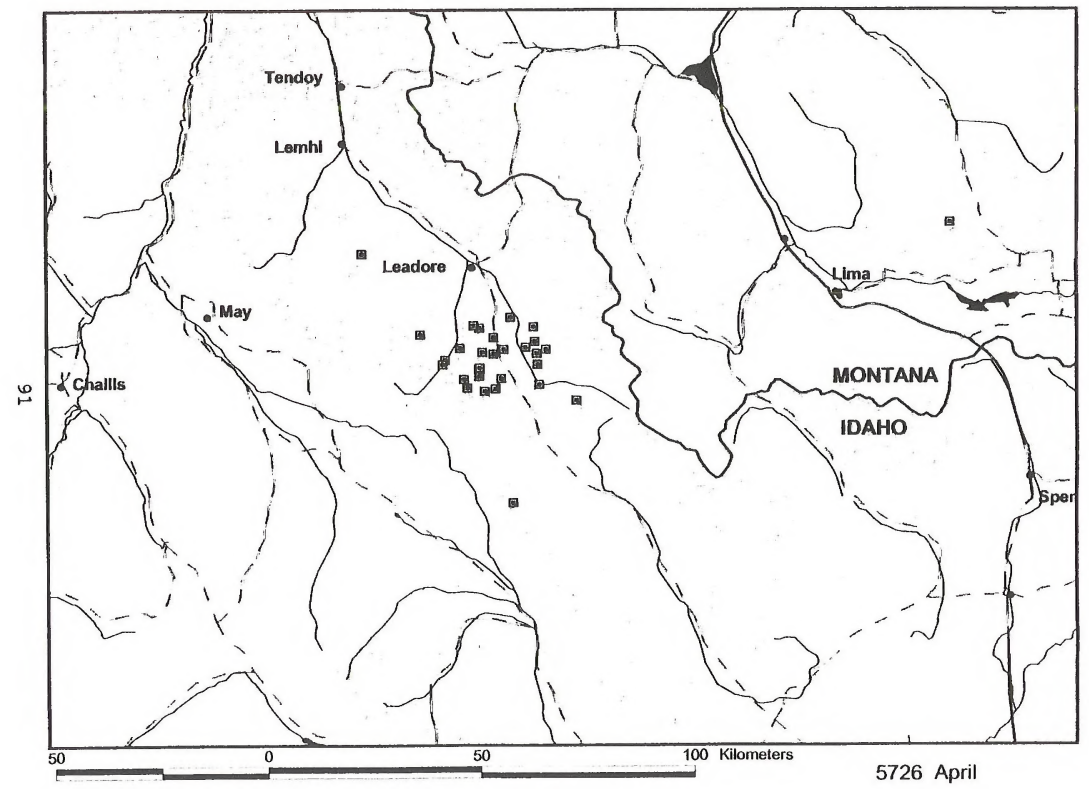




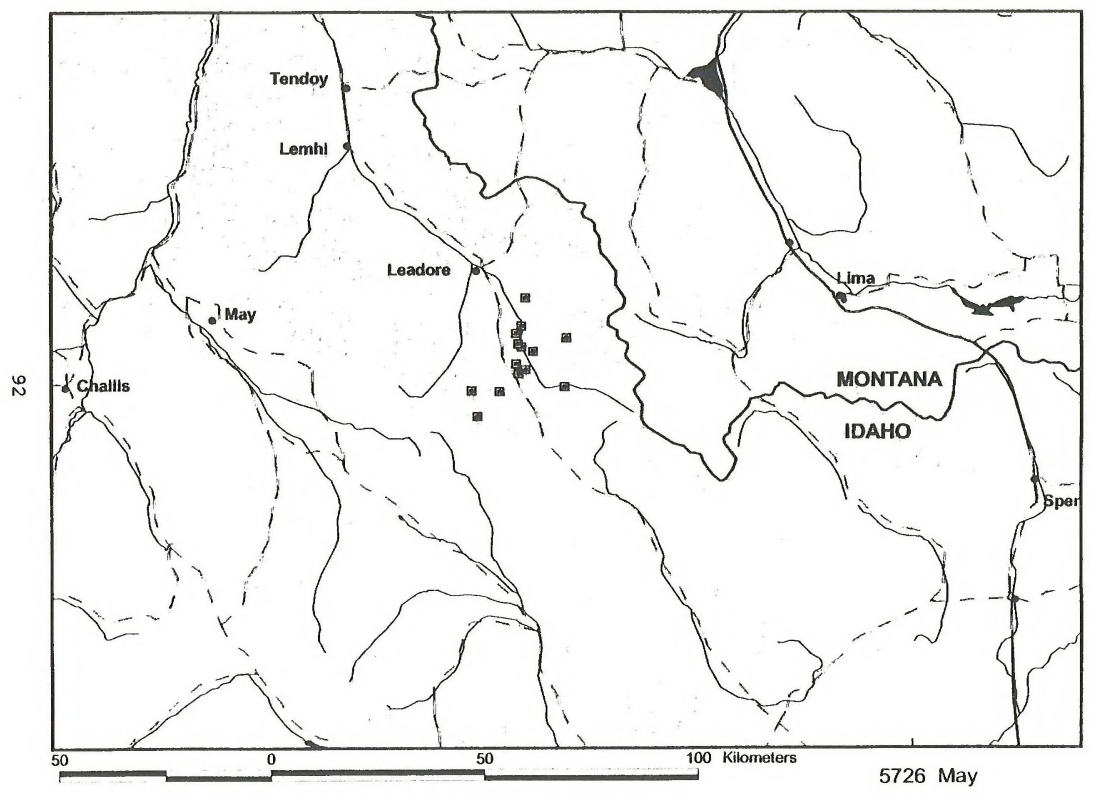

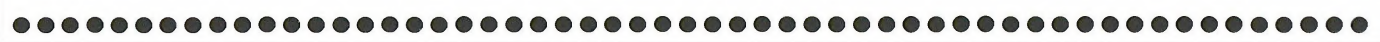




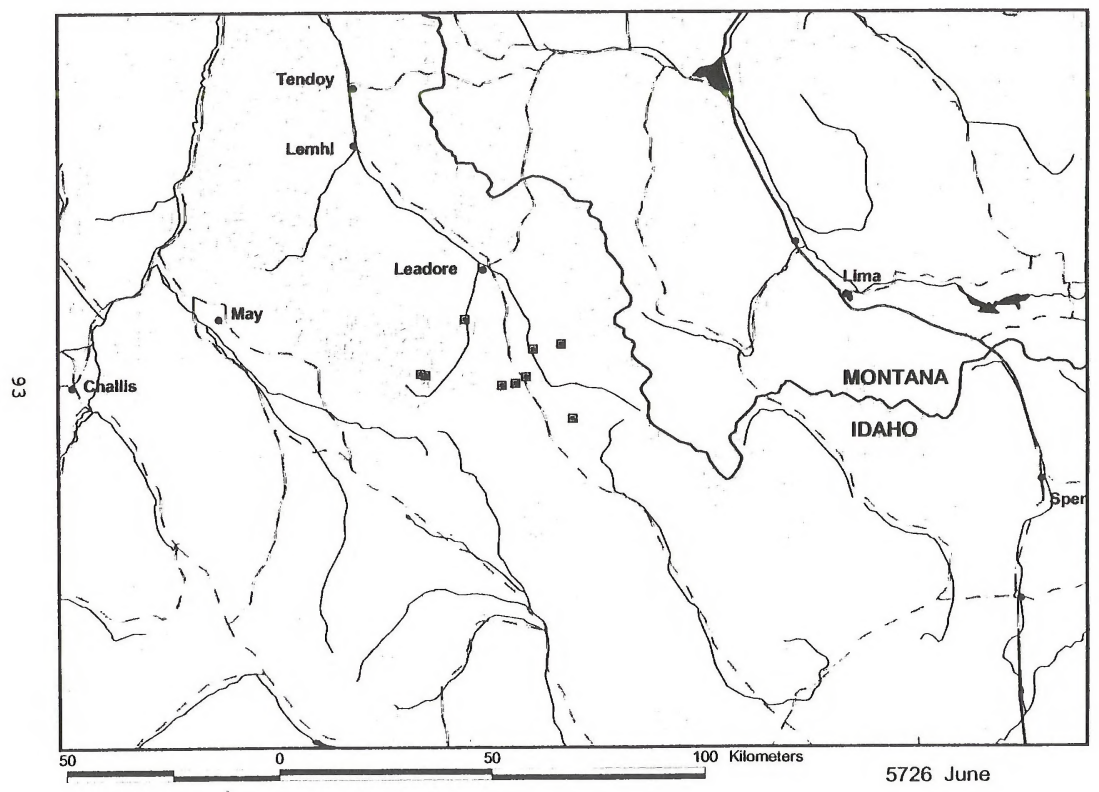




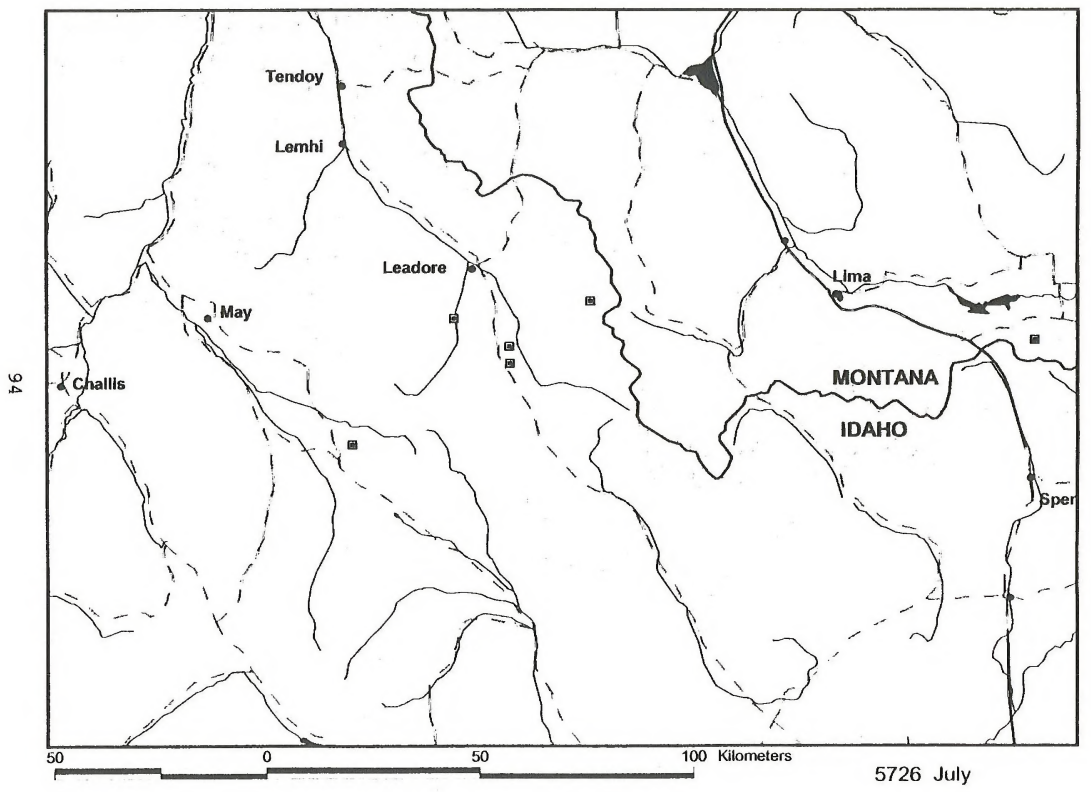

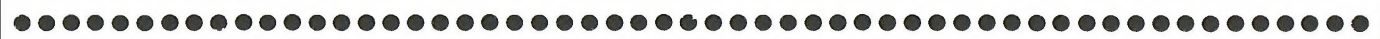




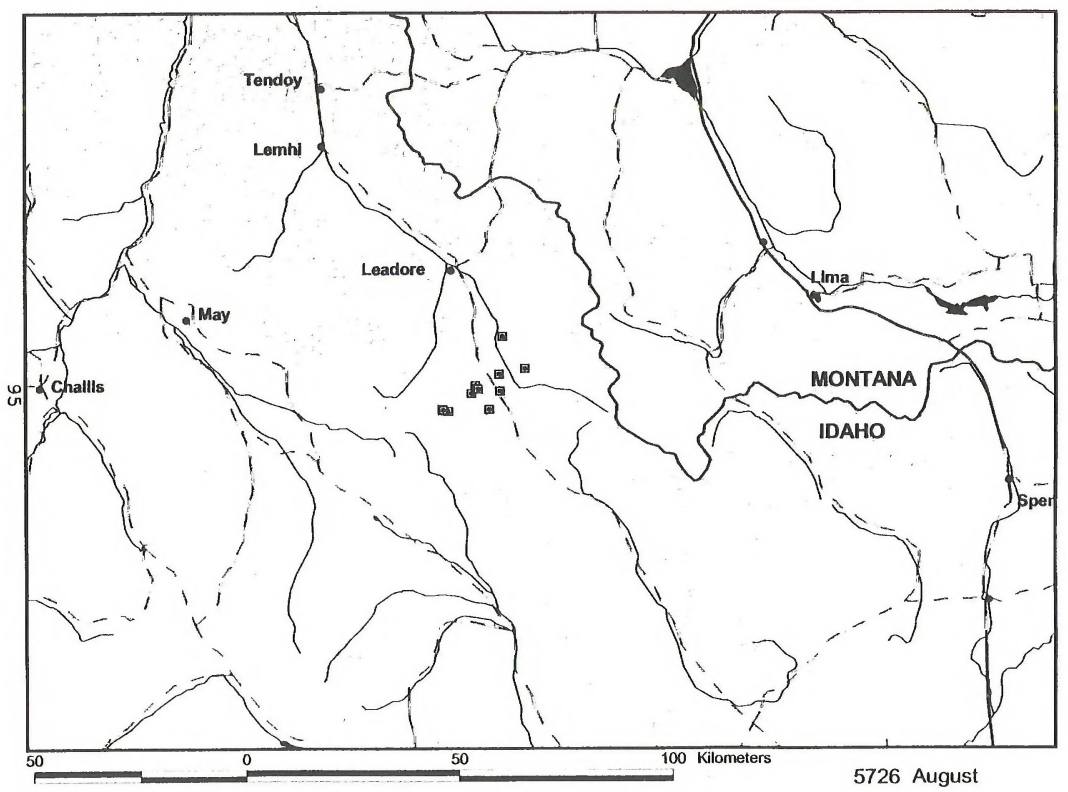




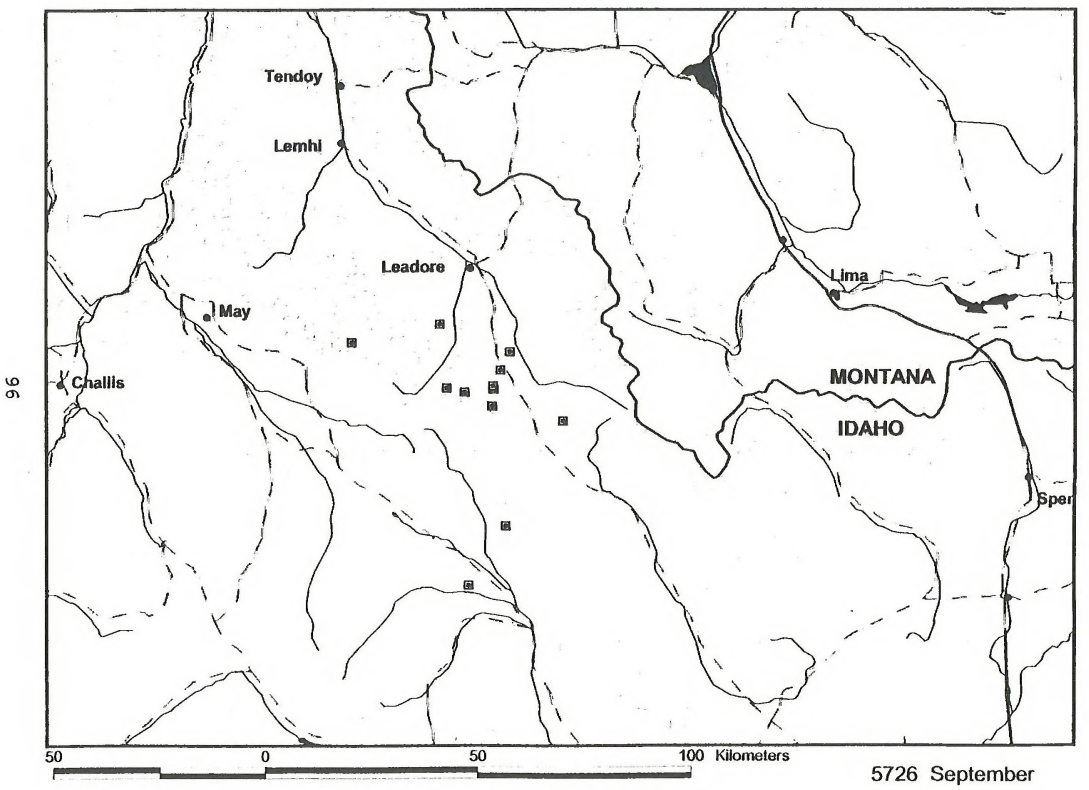

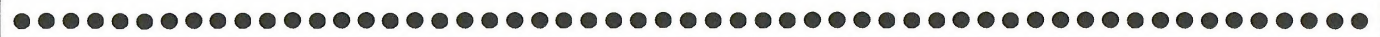




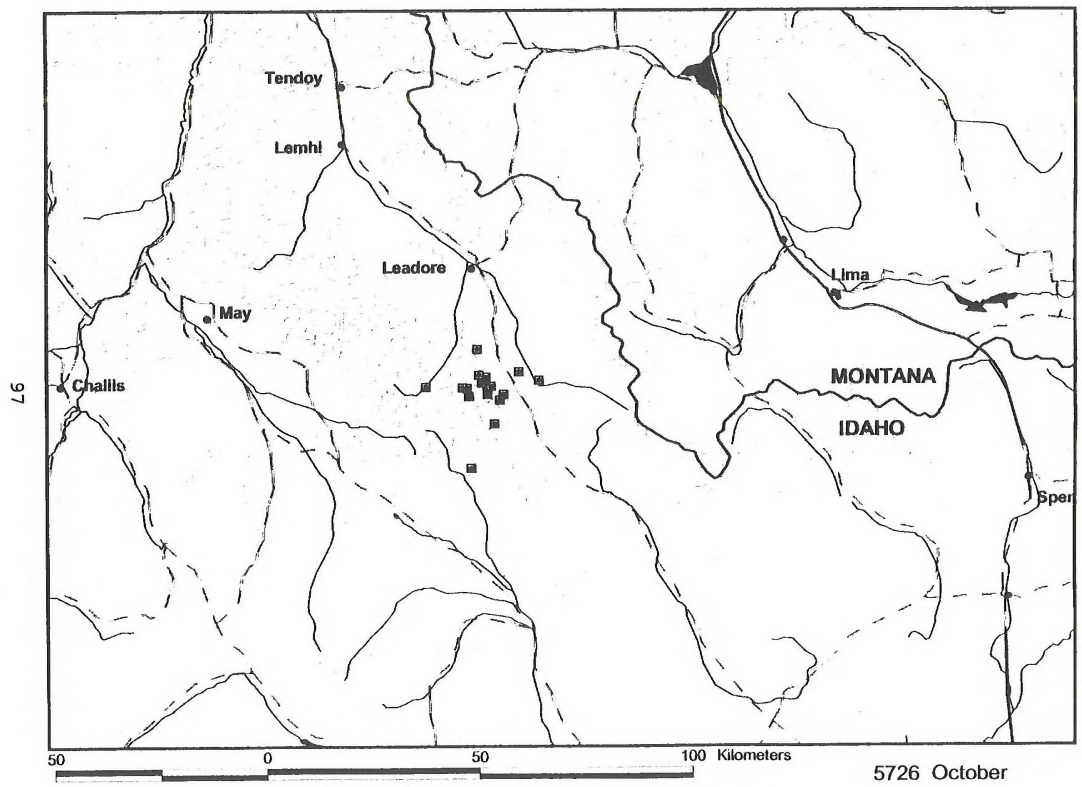




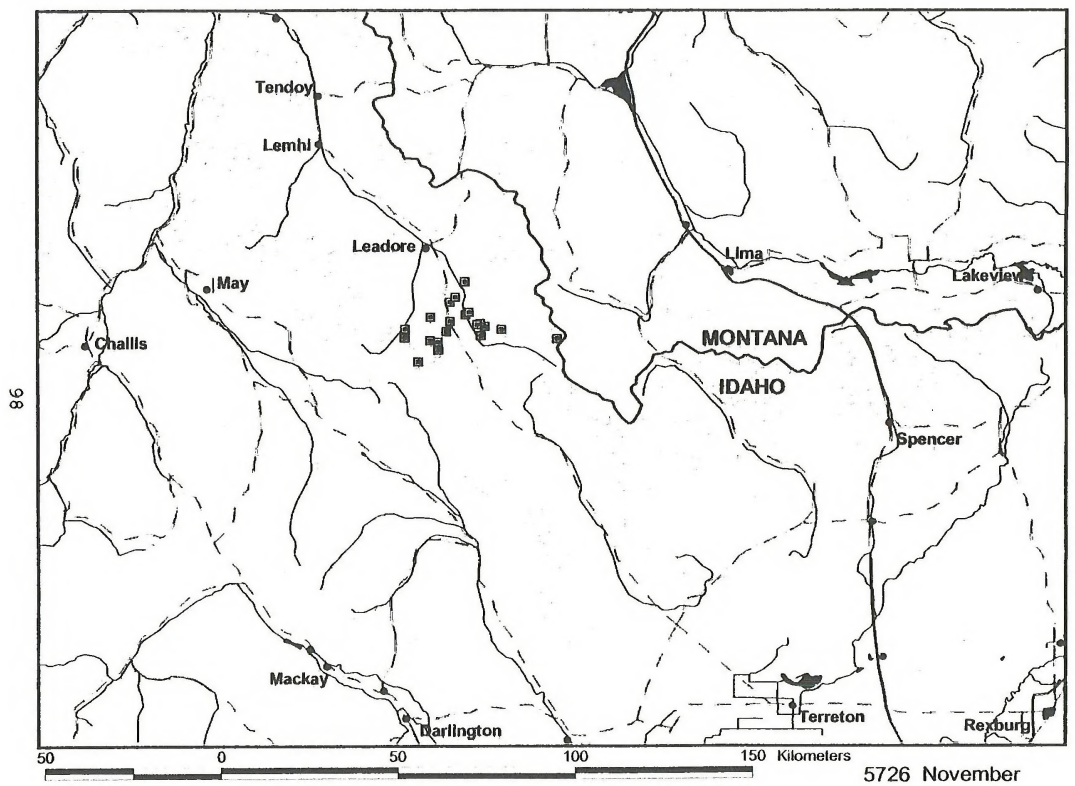

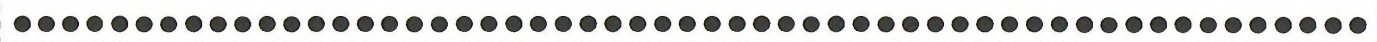




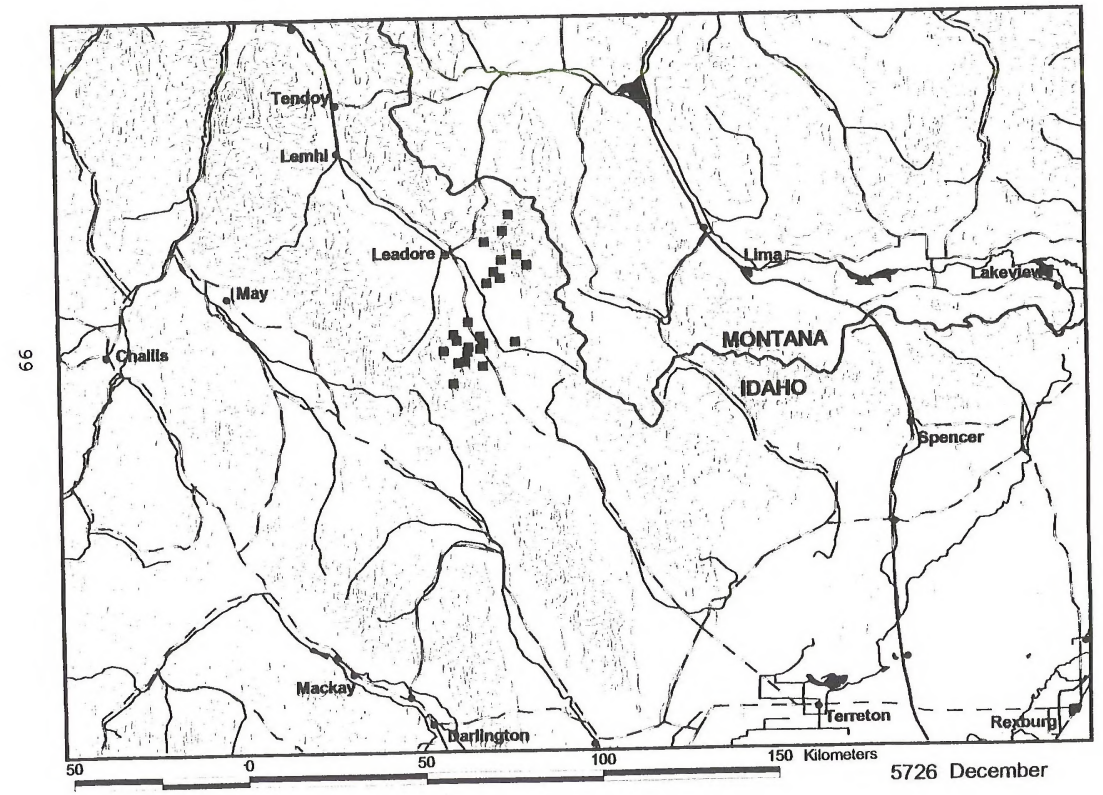


Monthly range maps for subadult, male golden eagle 5699; January through May 1996. 


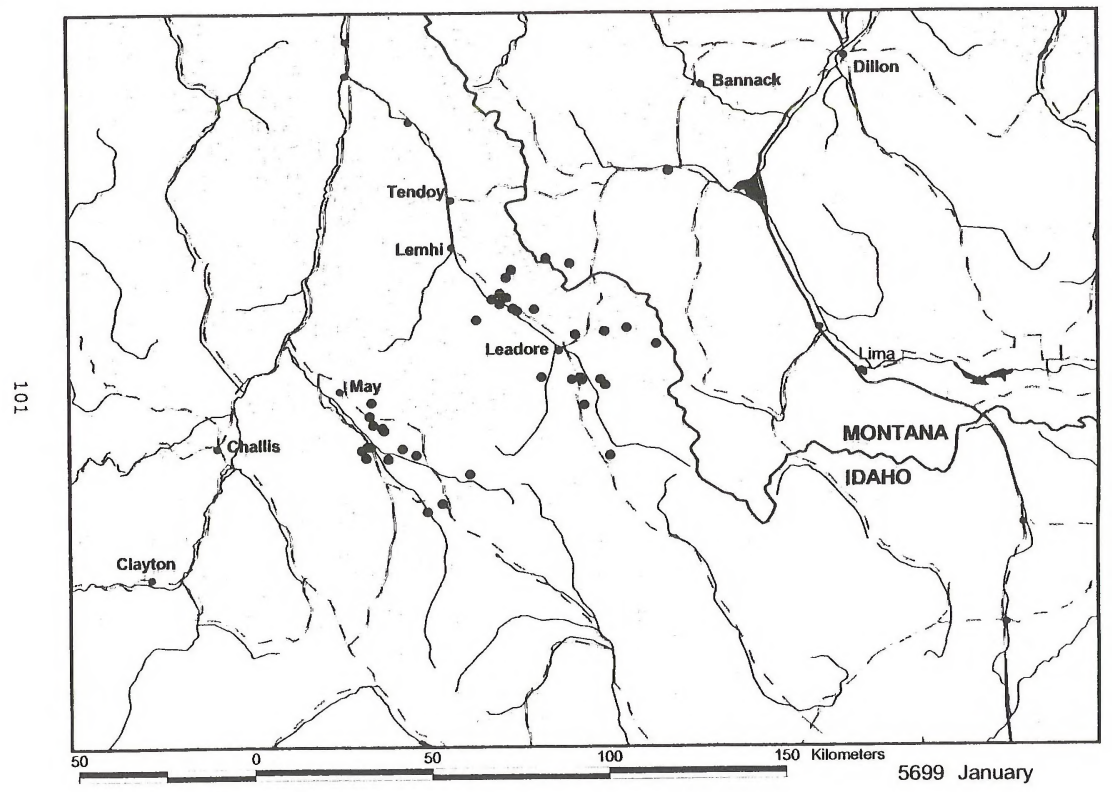




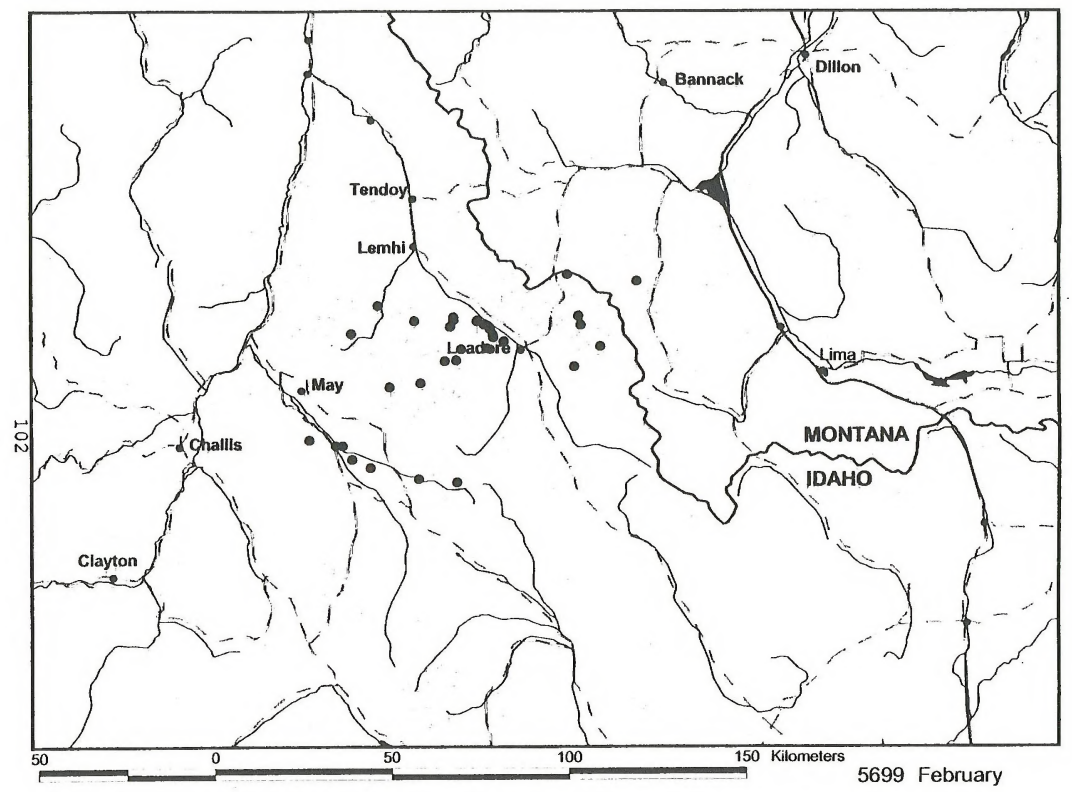

00000000000000000000000000000000000000000000000000000 


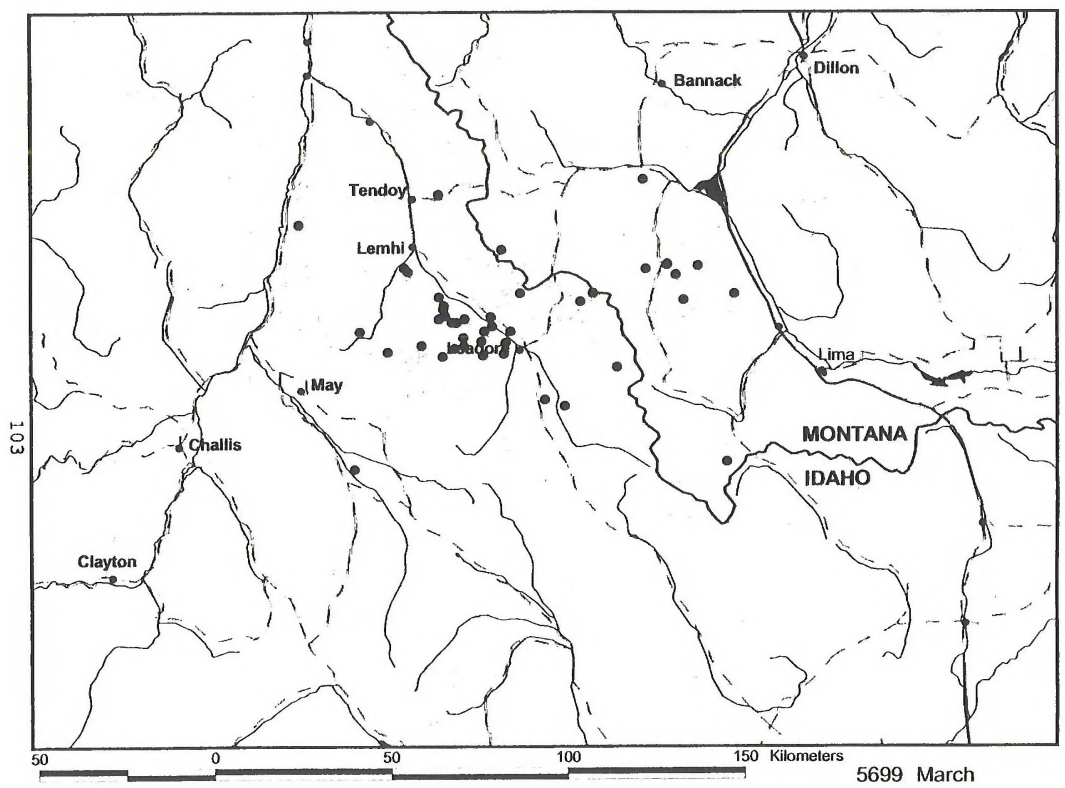




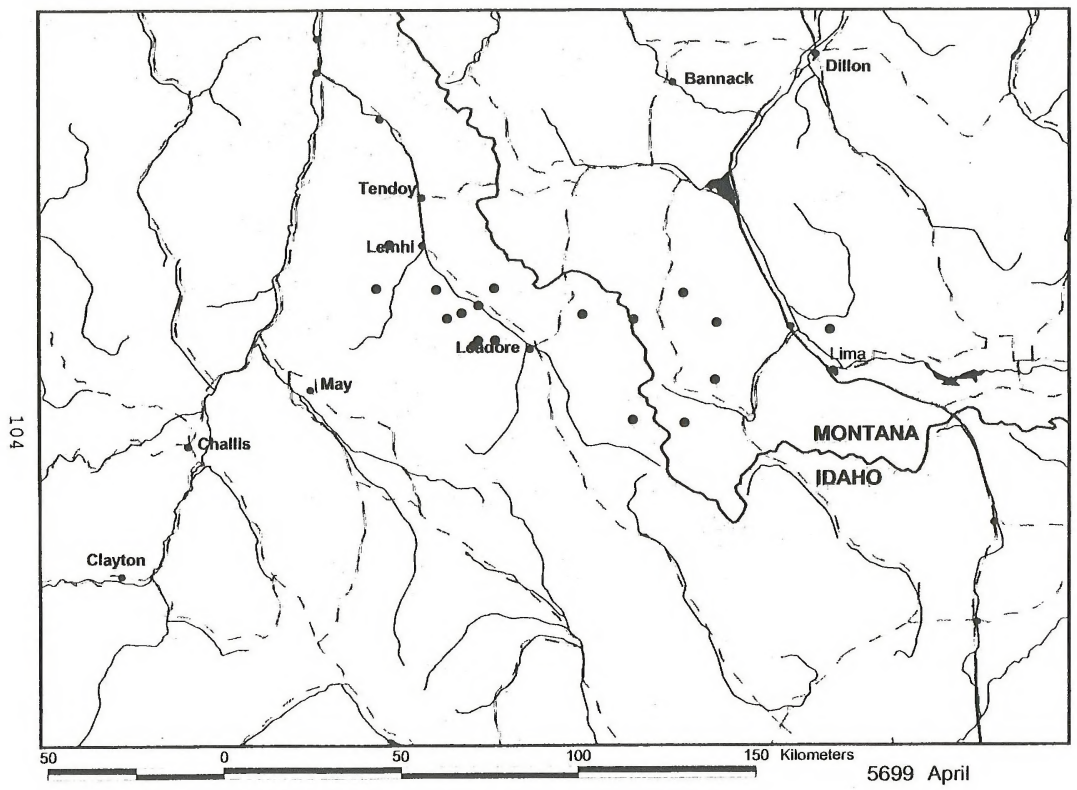

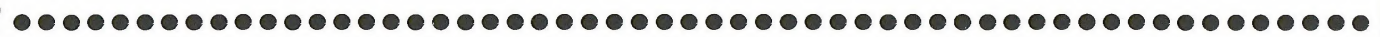




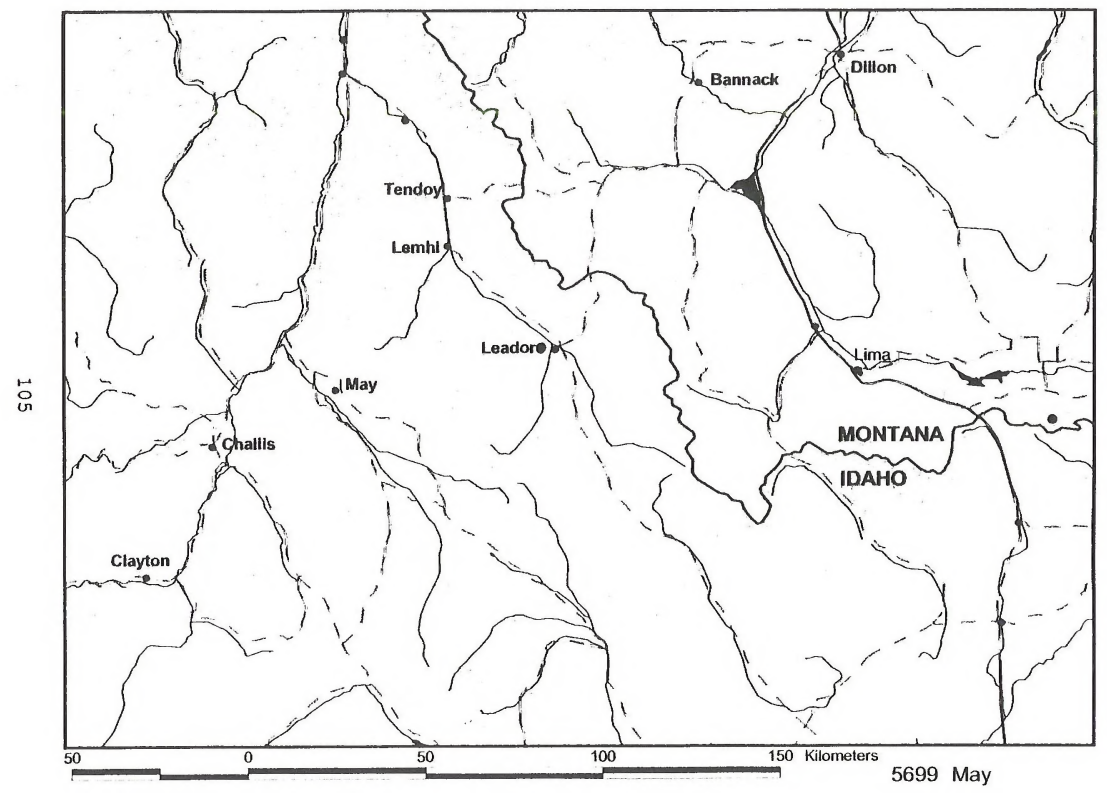


Monthly range maps for subadult, male golden eagle 5738 ; January through November 1996. 


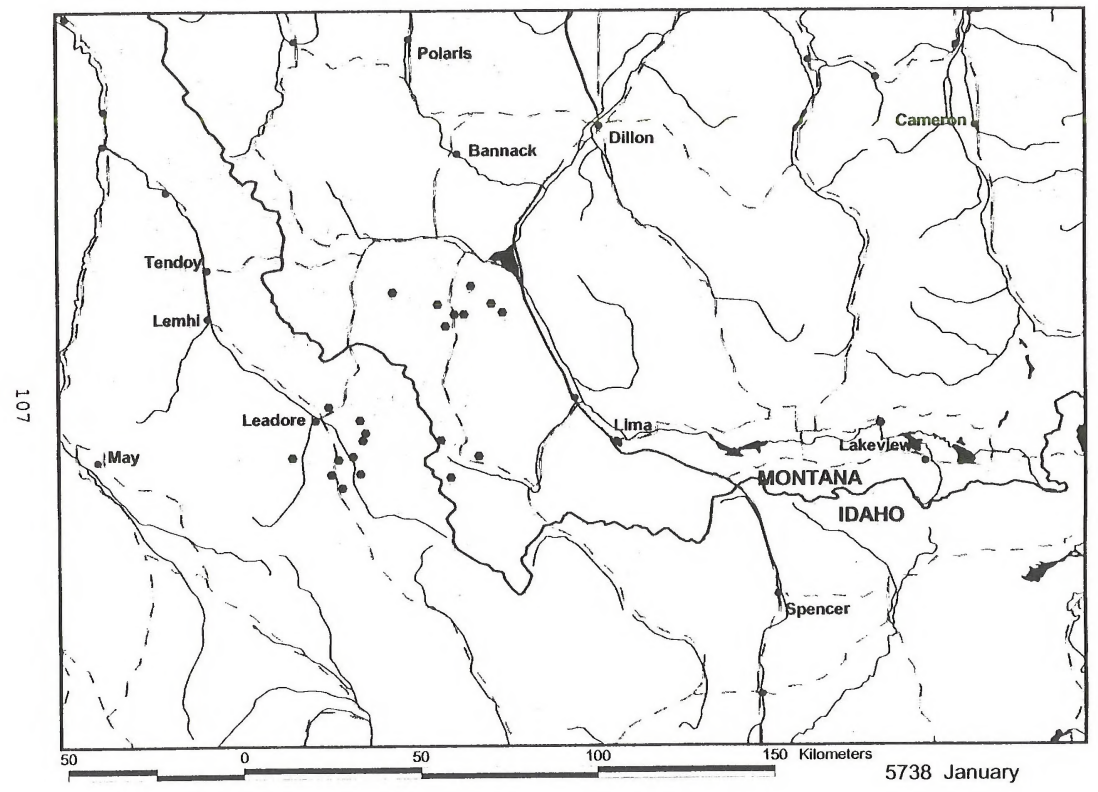




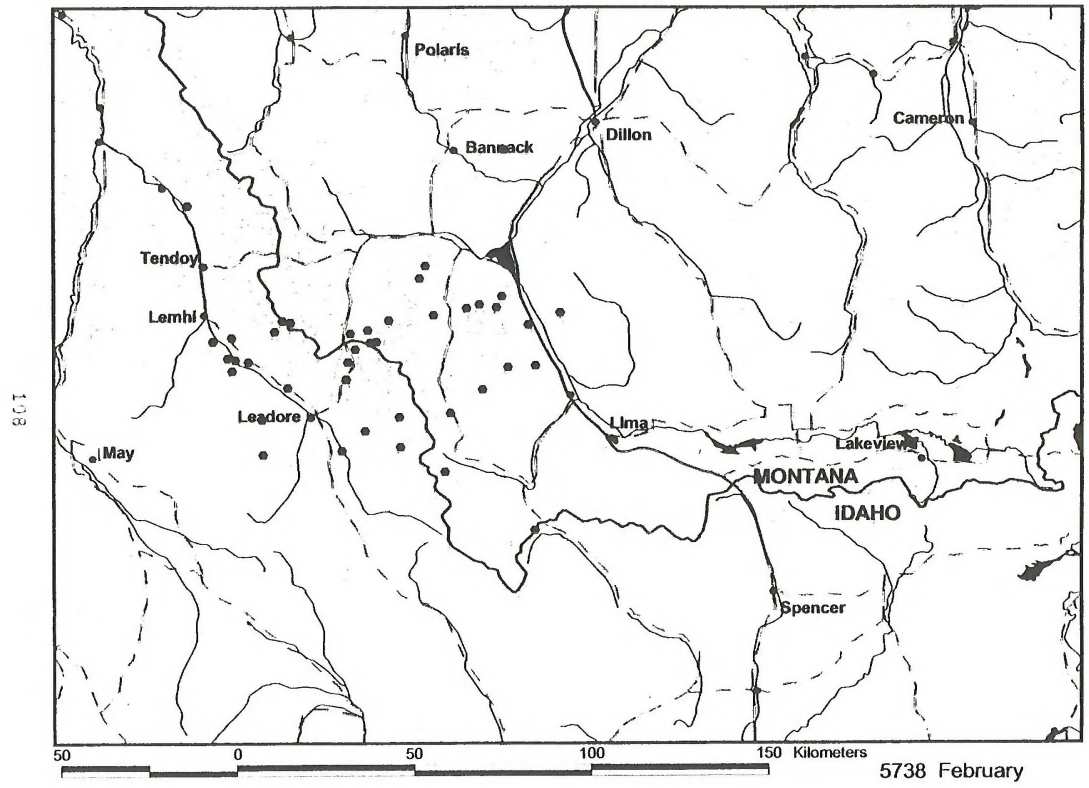

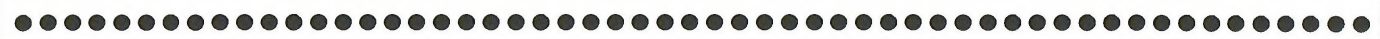




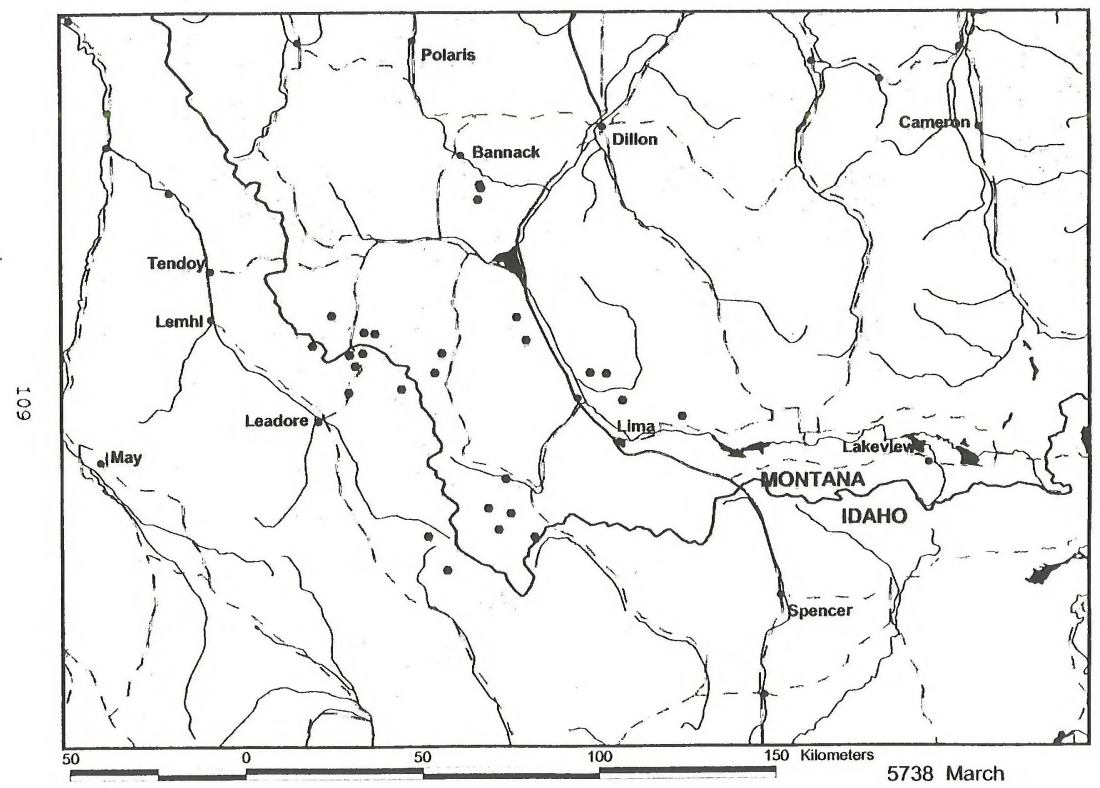




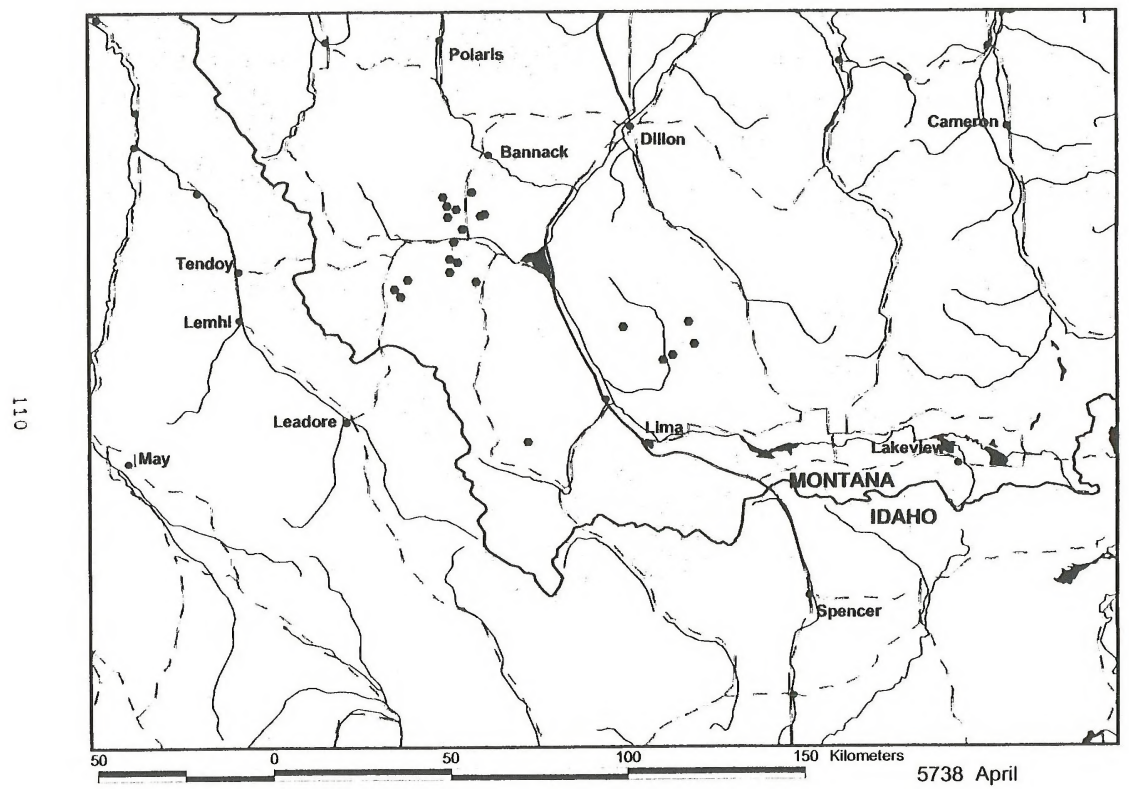

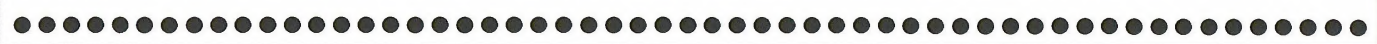




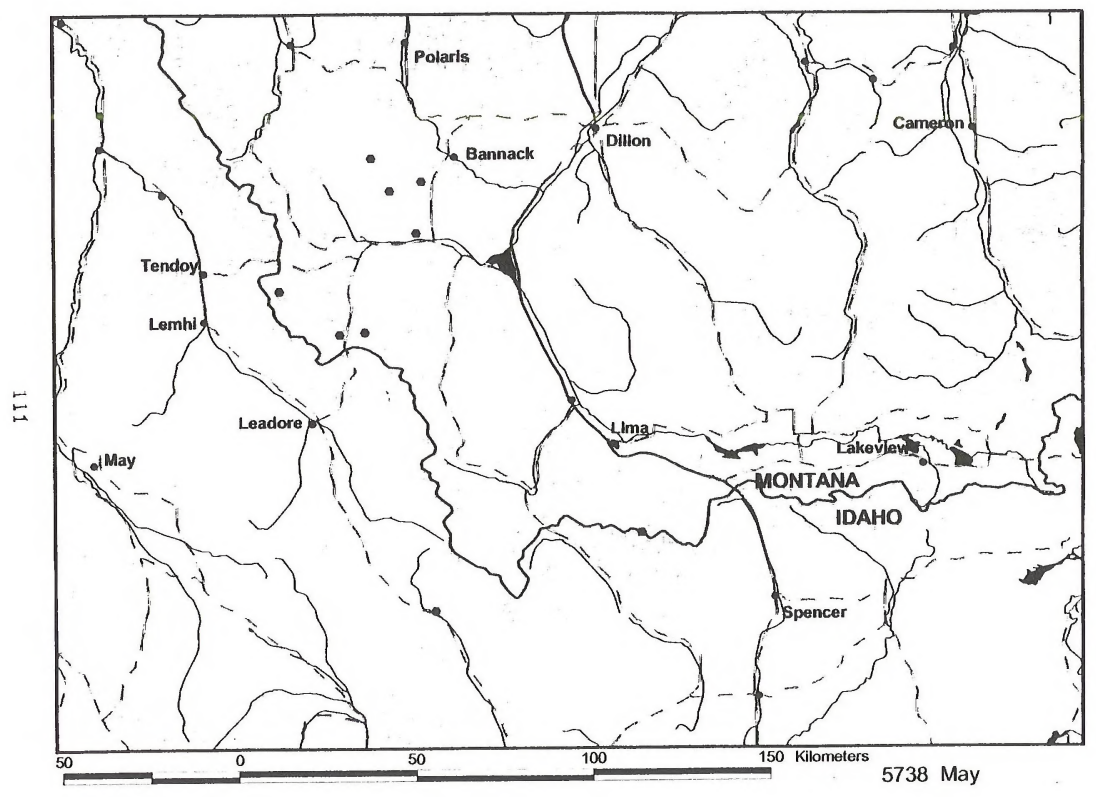




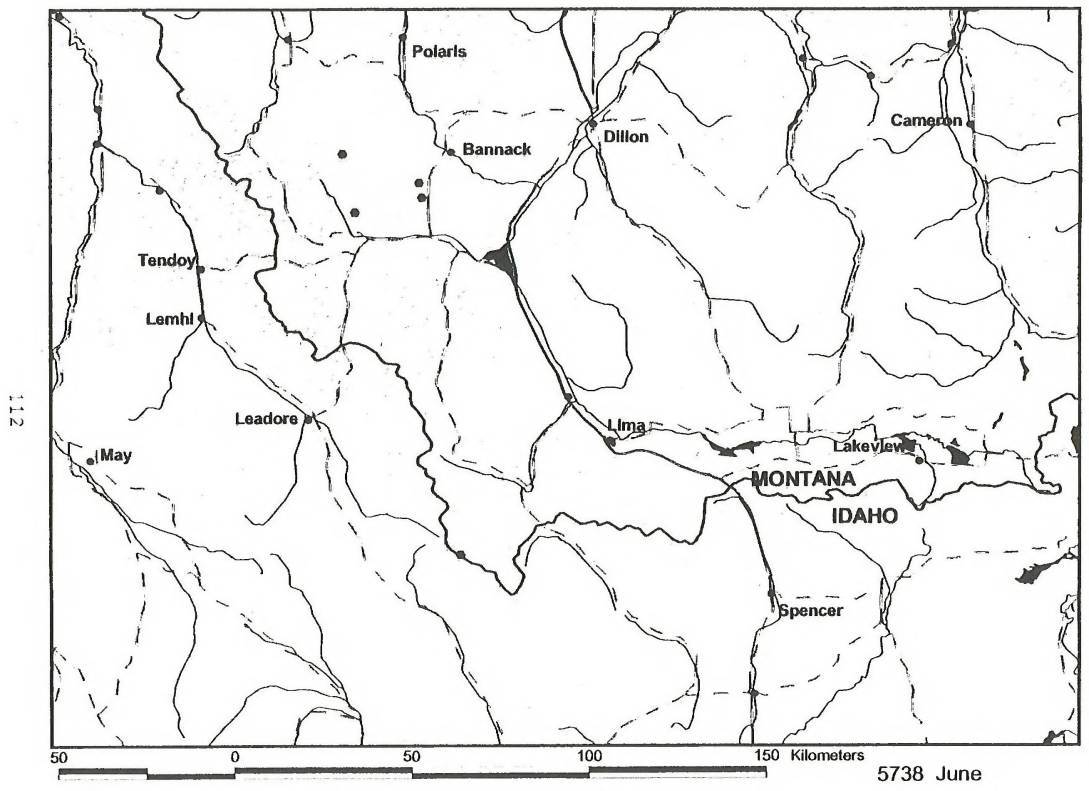

0000000000000000000000000000000000000000000000000000000 


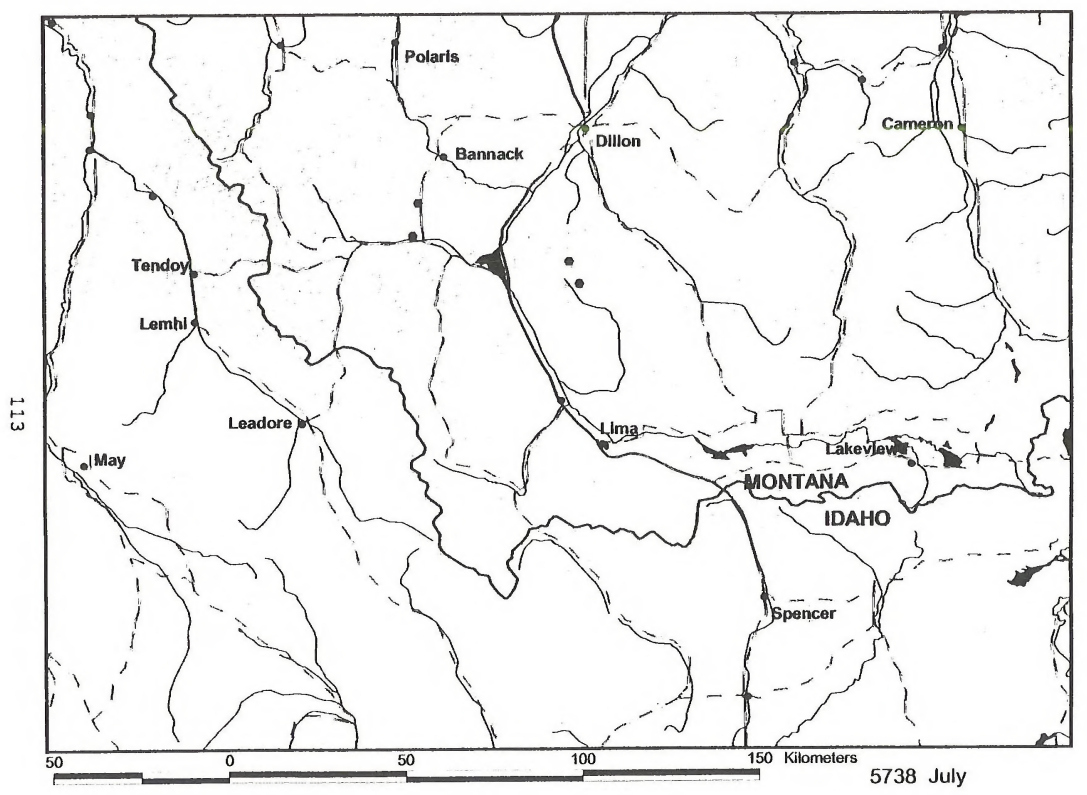




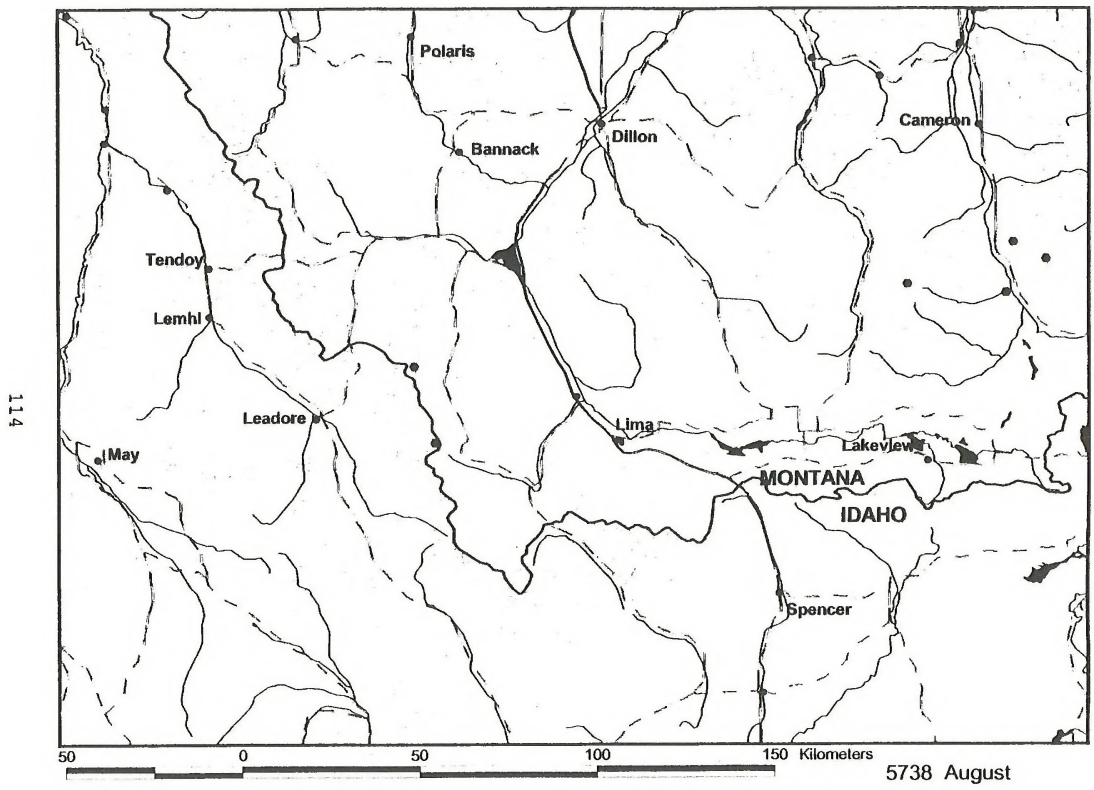

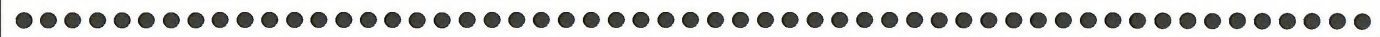




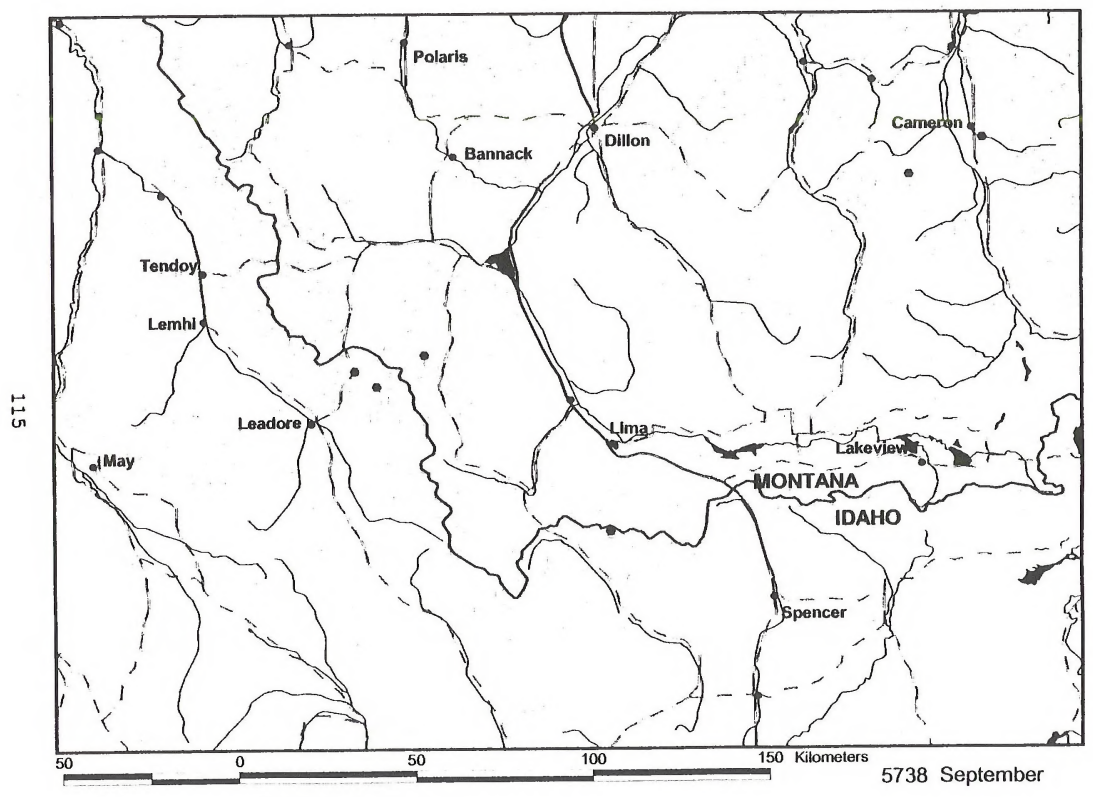




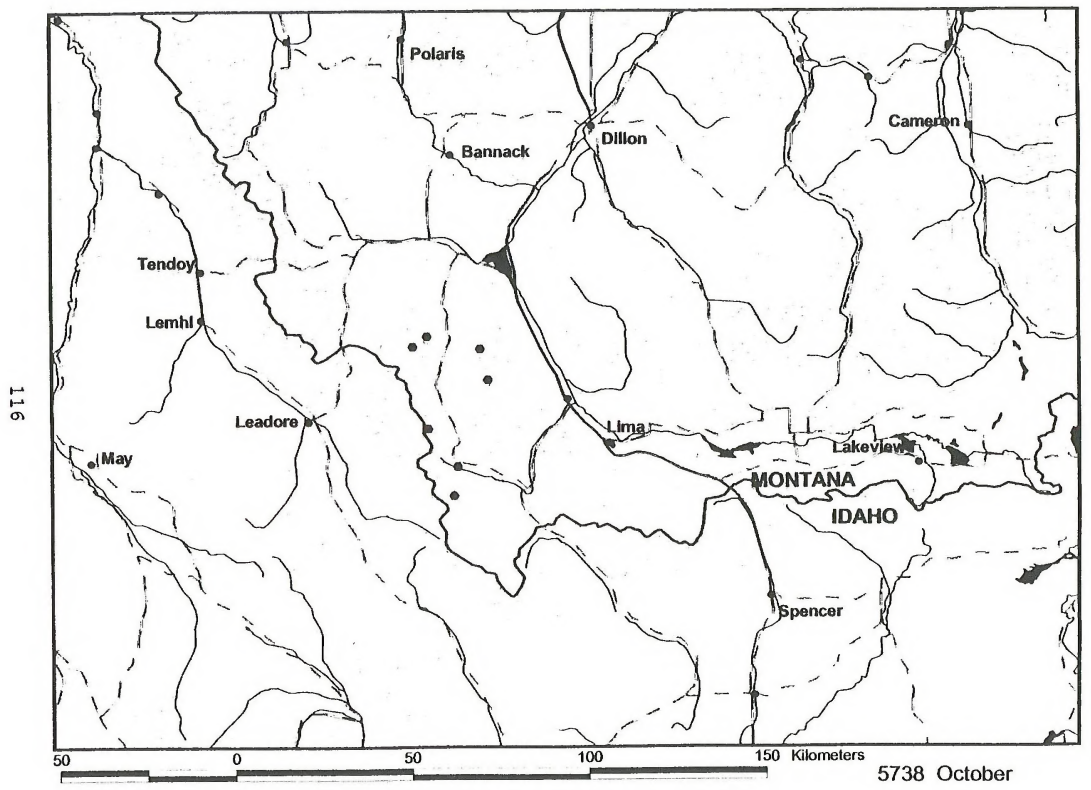

000000000000000000000000000000000000000000000000000 


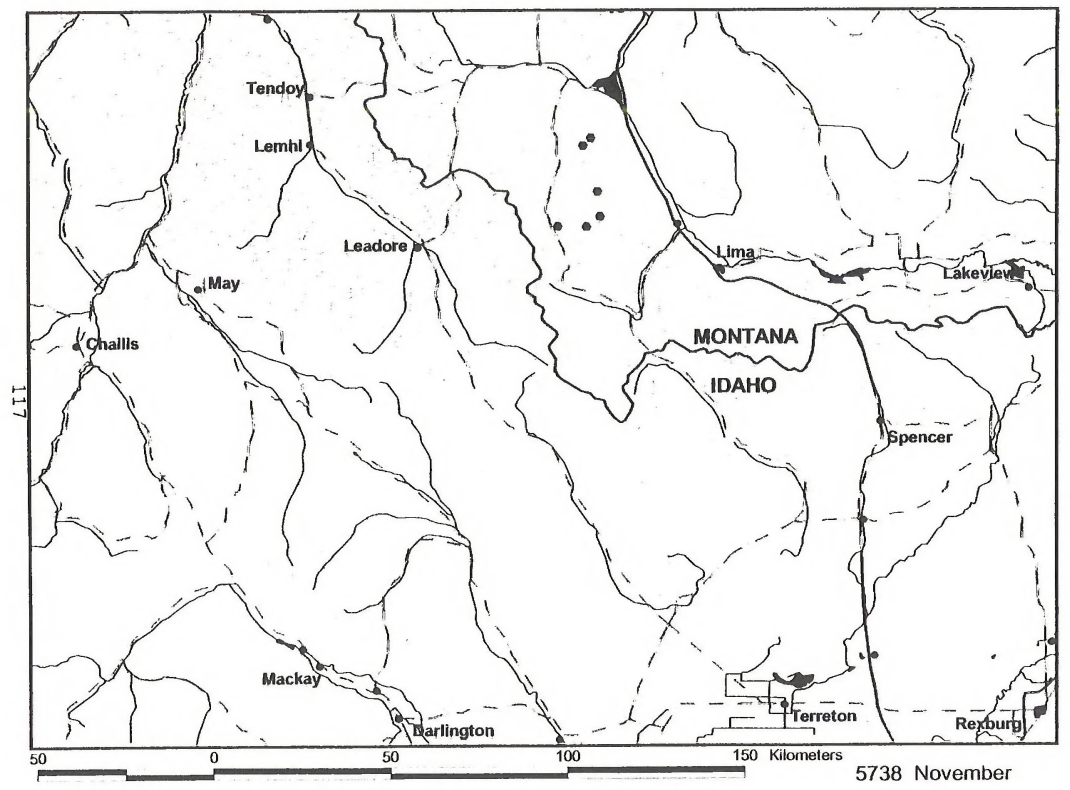


Monthly range maps for subadult, female golden eagle 5707; January through October 1996. 


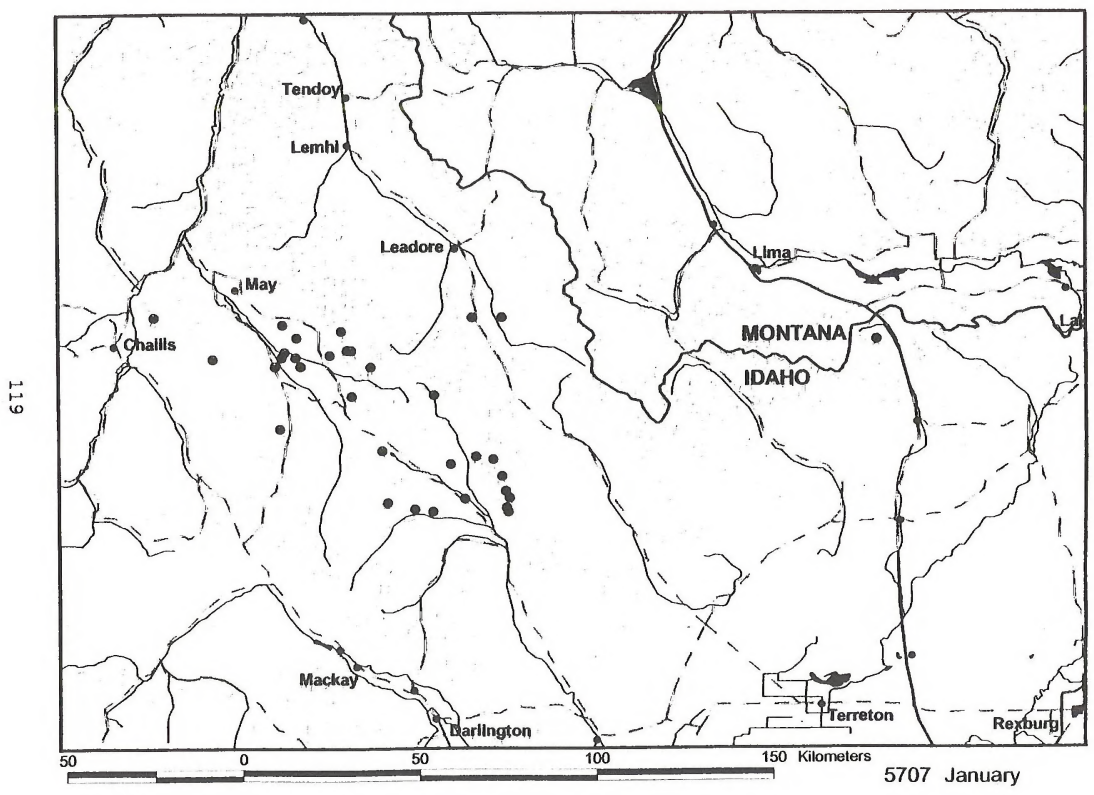




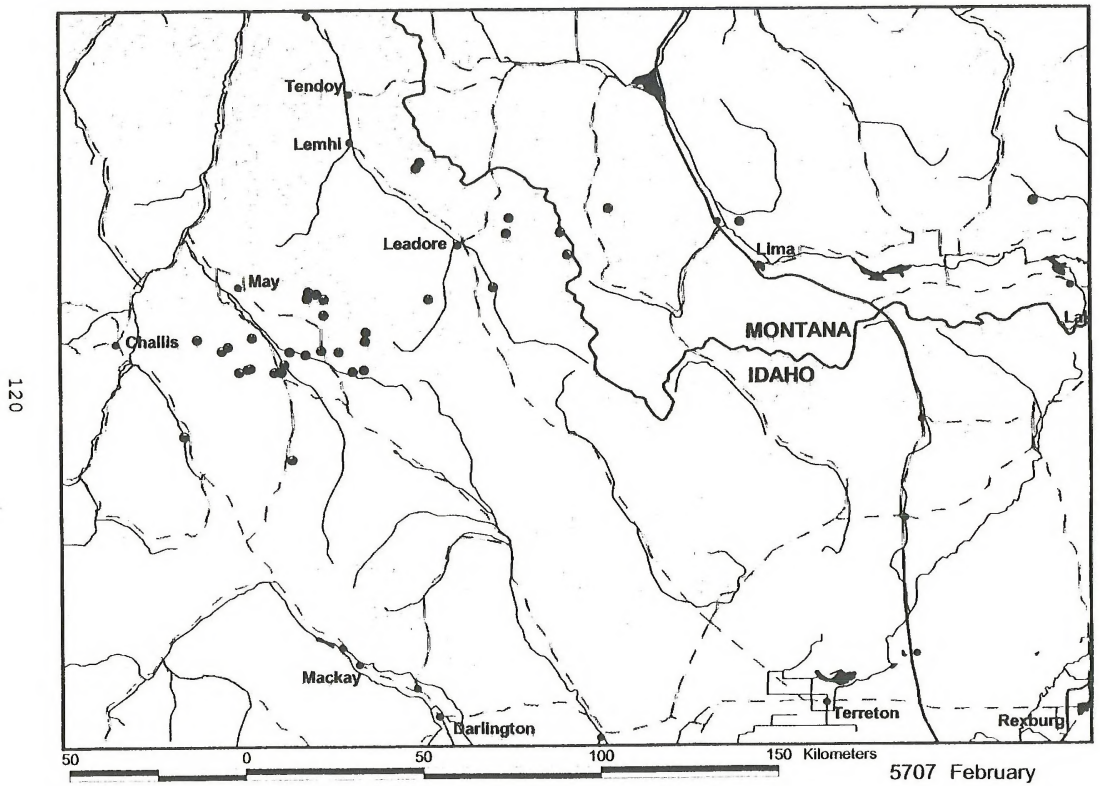

00000000000000000000000000000000000000000000000000000 


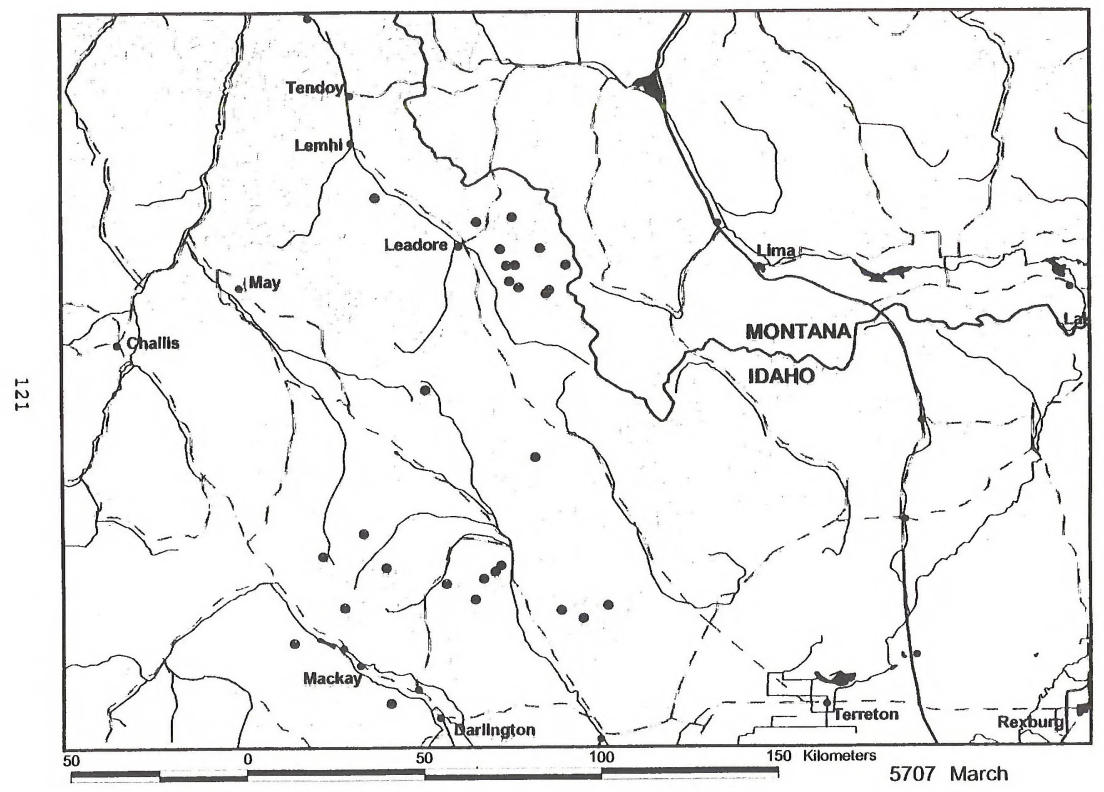




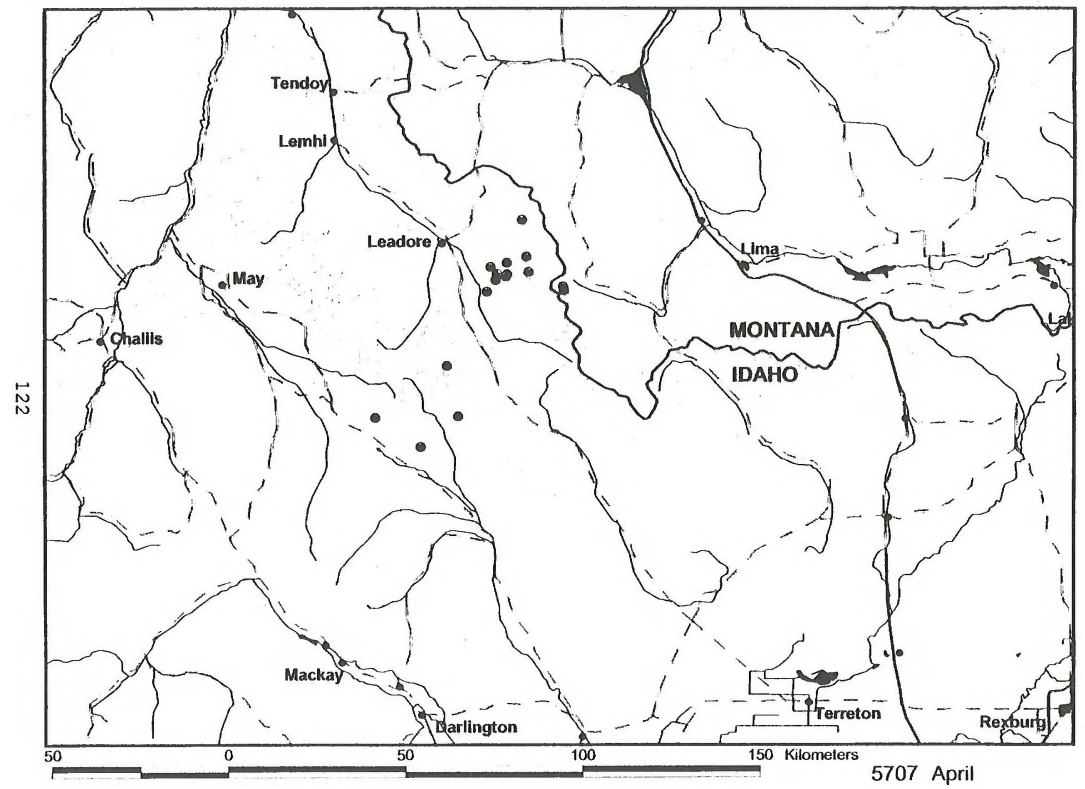

0000000000000000000000000000000000000000000000000000000 


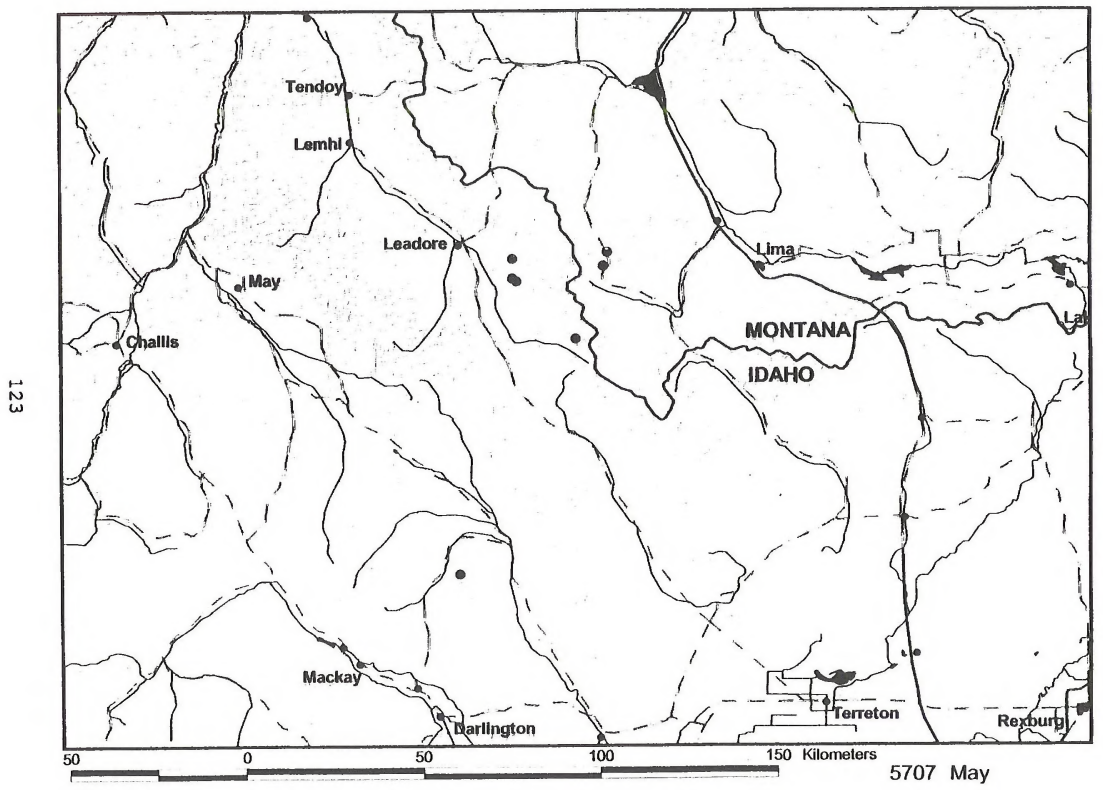




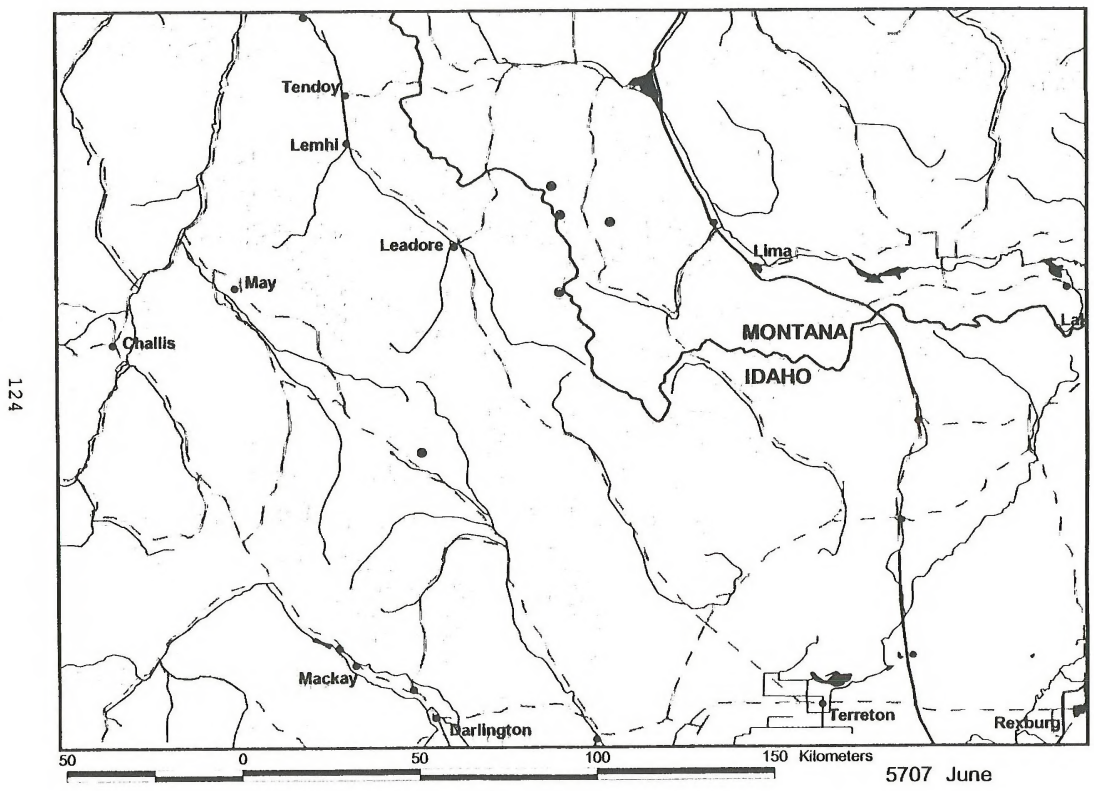

0000000000000000000000000000000000000000000000000000000 


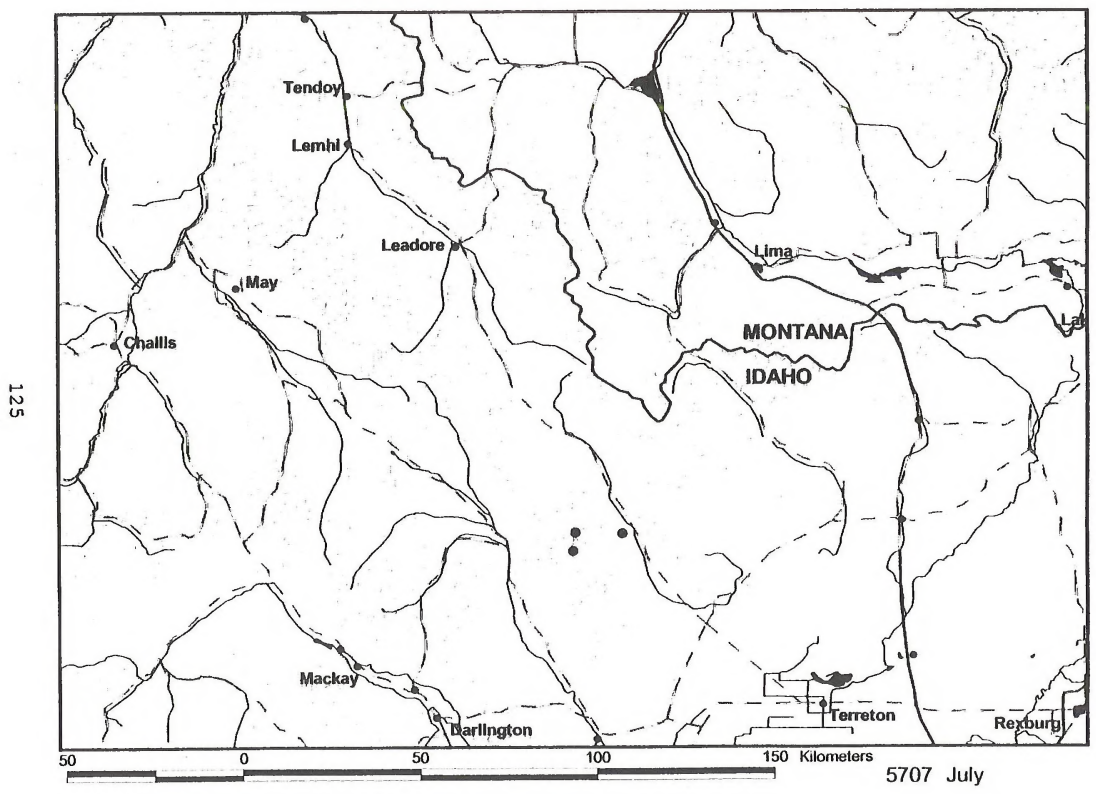




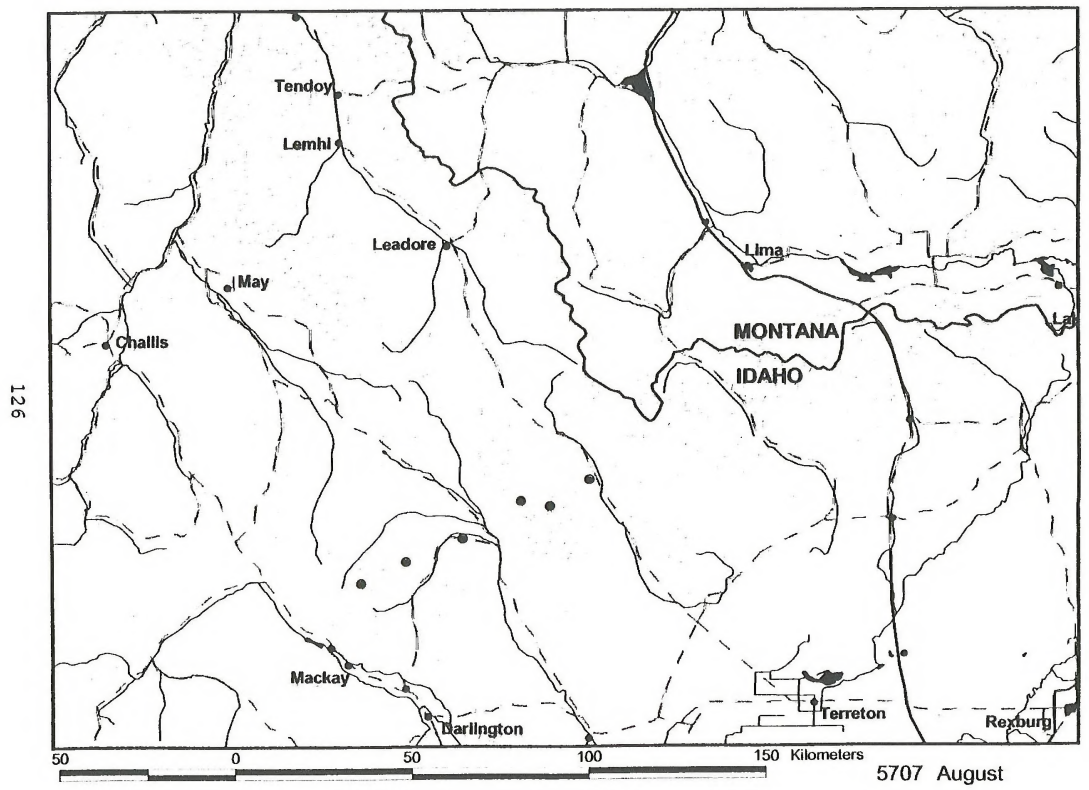

000000000000000000000000000000000000000000000000000000 


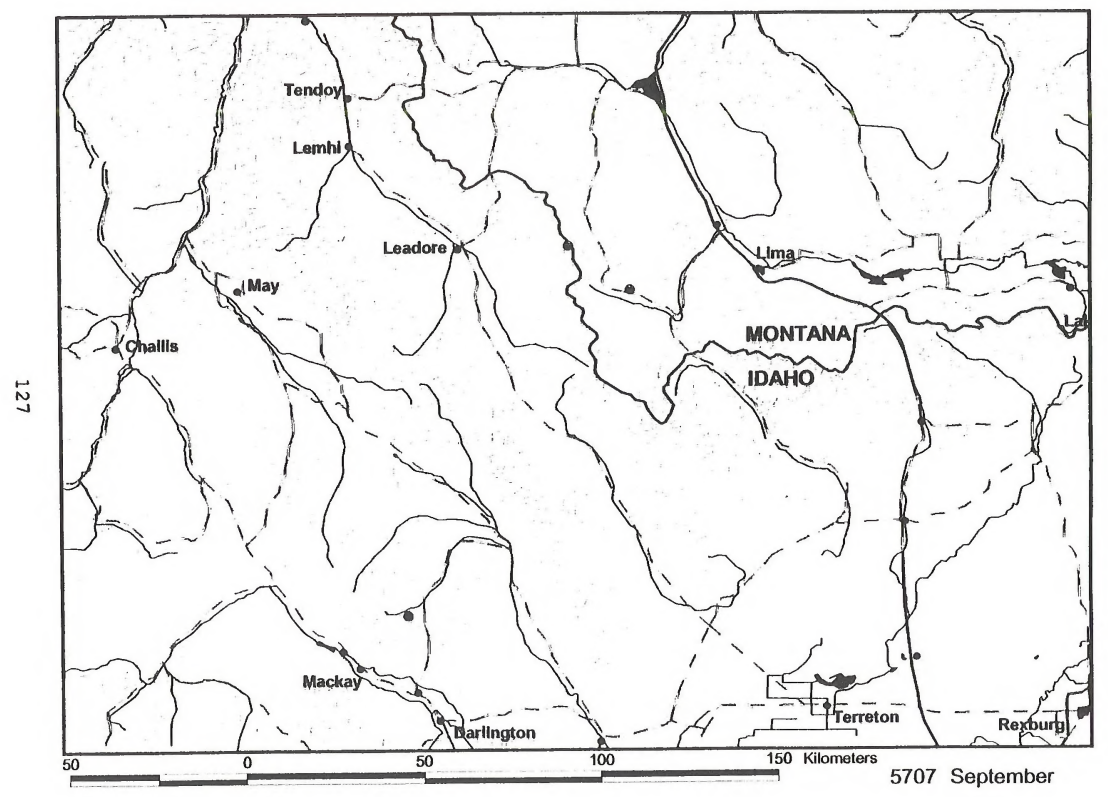




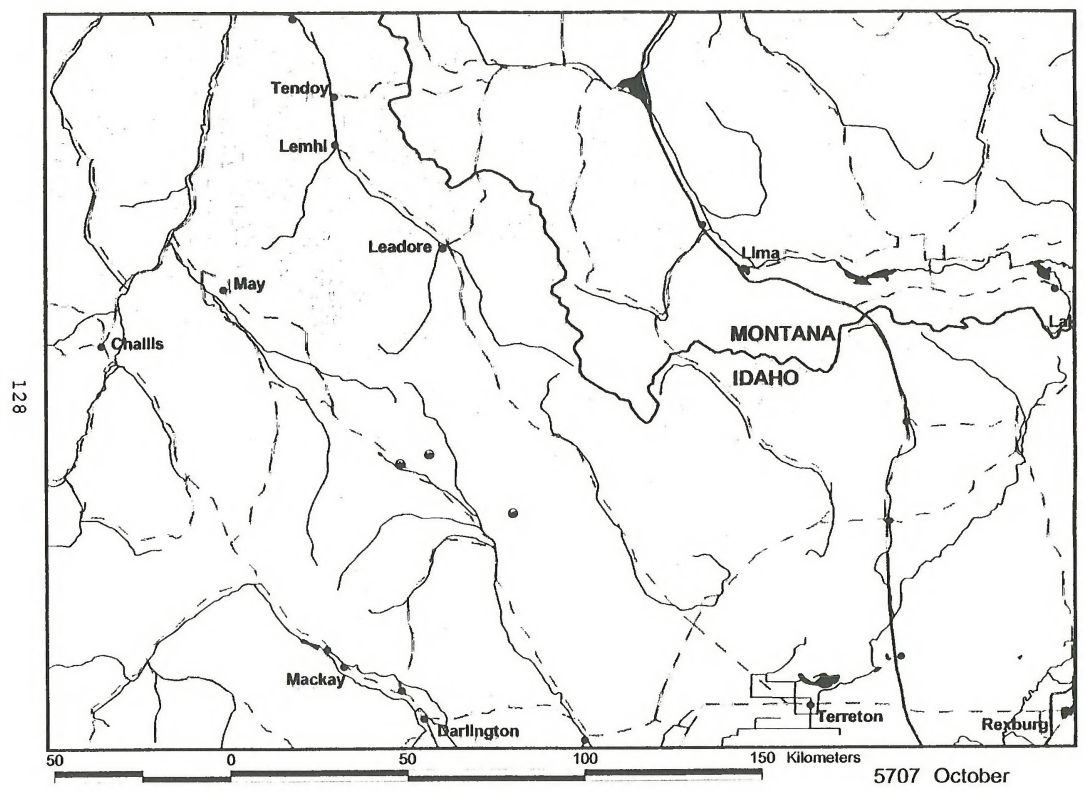

00000000000000000000000000000000000000000000000000000 

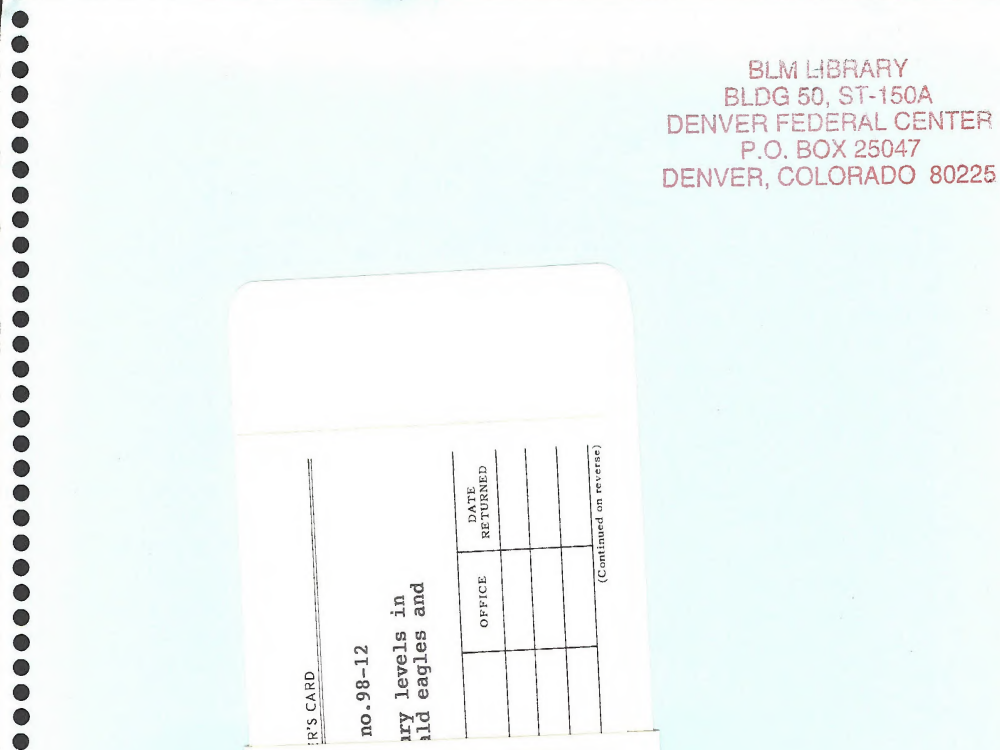

OL $84.2 . \pm 352$ no.98-12

88055063

lead and mercury levels in golden and bald eagles and

BLDG 50, ST-150A

DENVER FEDERAL CENTEF

P.O. BOX 25047

DENVER, COLORADO 80225 
